Equações diferenciais funcionais neutras, comportamento assintótico e representação

\author{
Patricia Hilario Tacuri
}



SERVIÇO DE PÓS-GRADUAÇÃO DO ICMC-USP

Data de Depósito:

Assinatura:

\section{Equações diferenciais funcionais neutras, comportamento assintótico e representação}

Patricia Hilario Tacuri

Orientador: Prof. Dr. Miguel Vinícius Santini Frasson

Tese apresentada ao Instituto de Ciências Matemáticas e de Computação - ICMC-USP, como parte dos requisitos para obtenção do título de Doutor em Ciências - Matemática. VERSÃO REVISADA.

USP - São Carlos

Fevereiro de 2013 
Ficha catalográfica elaborada pela Biblioteca Prof. Achille Bassi e Seção Técnica de Informática, ICMC/USP, com os dados fornecidos pelo(a) autor(a)

Tacuri, Patricia Hilario
T115e Equações diferenciais funcionais neutras, comportamento assintótico e representação / Patricia Hilario Tacuri; orientador Miguel Vinícius Santini Frasson. -- São Carlos, 2012. $150 \mathrm{p}$.

Tese (Doutorado - Programa de Pós-Graduação em Matemática) -- Instituto de Ciências Matemáticas e de Computação, Universidade de São Paulo, 2012.

1. Equações diferenciais funcionais neutras. 2. Comportamento assintótico. 3. EDOs generalizadas. 4. EDFNs em medida. 5. EDFNs periódicas. I. Frasson, Miguel Vinícius Santini, orient. II. Título. 
A mis queridos padres Donato

y Guillermina, con mucho amor. 



\section{Agradecimentos}

Ao concluir mais uma etapa da minha vida acadêmica, minha imensa gratidão a Deus, por dar-me forças a cada dia para não desistir, pelo amor que me faz acreditar num futuro melhor.

Agradezco de manera muy especial a mis queridos padres Guillermina y Donato, por enseñarme que las matemáticas estan presentes en nuestro día a día, por incentivarme siempre a dar lo mejor de mí, por apoyar mis decisiones y comprender mi ausencia necesaria para mi crecimiento profesional . A mi hermano Alexander, por el ejemplo de superación y dedicación al trabajo, por las palabras de aliento, por la confianza que me hace sentir su hermana mayor.

Ao Norbil Cordova, meu namorado, pelo apoio incondicional ao longo desta jornada, pelas palavras de estímulo que me ajudarem a superar as dificuldades na minha vida, pelo carinho, paciência, compreensão e pelo "presente" que me deu, o seu amor. Amo-te muito.

Ao meu orientador, Prof. Miguel Frasson, pela valiosa contribuição na minha formação profissional através dos conhecimentos transmitidos, pela dedicação e especial atenção nesses anos de orientação, pela disponibilidade do seu tempo para a realização deste trabalho, pelas orientações sobre a vida, pela amizade e confiança que me passou mediante as suas palavras. Agradeço também à sua linda família. Obrigada por tudo.

À Prof ${ }^{a}$. Marcia Federson e Jaqueline Mesquita, pela contribuição numa parte deste trabalho, pelas palavras demonstrando seu apoio e incentivo a melhorar, mostrando-me uma série de oportunidades, pela amizade com a qual me bridaram e me fizeram sentir em casa.

À banca examinadora pelas sugestões apresentadas.

Aos meus amigos e colegas do ICMC, Alisson, Ana Paula, André, Eber, Eduard, Fabio, Flor Karina, Fernando, Gabriel, Giselle, Iris, Jhon, José, Jorge, Jorge Alberto, Luis Renato, Manuel, Maria Carolina, Maribel, Matheus, 
Mostafa, Nancy, Nazira, Paulo, Pedro, Pooya, Rawlisson, Suzete e Taciana, pelo companheirismo durante esta etapa. Às meninas da CPG, Ana Paula, Carolina, Glaucia, Laura, Lhaís pela atenção dedicada nos assuntos burocráticos necessários.

Aos meus amigos, minha família em São Carlos, Renato Alejandro, Roxana, Cecilia, Henry, Johana, Camilo, Nathali, Elizabeth, Niko, Lito e Katherine, pelo carinho que sempre me mostraram e pelas horas de descontração. Obrigada!

Aos meus amigos de Niterói, Campinas, Uberlândia, Sergipe e Recife, que ficaram na torcida ao longo de todos esses anos, obrigada pelo carinho transmitido através de seus e-mails e pela força que me transmitiram em cada telefonema, en especial, aos amigos, Cristhabel, Claudia Salas, Enrique, Marina, David, Claudia Luque, Michael, Nathali, Eduard, Richard, Edjane, Ricardo, Miguel e Clessius.

Aos meus professores da Universidad Nacional de San Agustin-Arequipa: Walter Torres Montes, pelo exemplo de dedicação ao ensino da matemática; Roberto Begazo Delgado, meu orientador na graduação; Vladimir Rosas; Javier Ordoñez e Richard Mamami. A todos eles agradeço pela contribuição na minha formação acadêmica.

Aos meus professores da Universidade Federal Fluminense, Dinamérico Pombo, Sebastião Firmo, Miriam Abdón, Juan Limaco, Gabriel Calsamiglia, Javier Ribón, Xu Cheng, Sergio Licanic pelo incentivo constante à pesquisa. Ao meu orientador durante o Mestrado, Prof. Haroldo Clark, pelos conhecimentos transmitidos e pela sua amizade.

Por fim, agradeço ao $\mathrm{CNPq}$ pelo apoio financeiro durante a execução deste trabalho. 


\section{Resumo}

O objetivo deste trabalho é investigar propriedades qualitativas das equações diferenciais funcionais neutras (EDFNs) e introduzir uma classe geral de equações chamadas EDFNs em medida.

Obtemos resultados sobre o comportamento assintótico para uma classe de EDFNs com coeficientes periódicos, onde o período e o retardamento estão racionalmente relacionados. Também, conseguimos mostrar que a dicotomia exponencial do operador solução das equações diferenciais funcionais com retardamento (EDFRs) não autônomas implica na existência de soluções limitadas para EDFRs não homogêneas associadas. Finalmente, através da teoria das equações diferenciais ordinárias generalizas (EDOs generalizadas), obtemos resultados de existência e unicidade, dependência contínua em relação aos dados inicias, das soluções das EDFNs em medida.

Os resultados novos apresentados neste trabalho estão contidos nos artigos $[31,43]$. 



\section{Abstract}

The aim of this work is to investigate qualitative properties of neutral functional differential equations (NFDEs) and introduce a general class of equations called measure NFDE .

We obtain results on the asymptotic behavior for a class of NFDEs with periodic coefficients, where the period and delay are rationally related. Moreover, we show that the exponential dichotomy of the solution operator of non autonomous retarded functional differential equations (RFDEs) implies the existence of bounded solutions to the associated non homogeneous RFDEs. Finally, using the theory of generalized ordinary differential equations (generalized ODEs), we obtain results of existence and uniqueness, continuous dependence on parameters of the solutions of measure NFDEs.

The new results presented in this work are contained in the articles [31,43]. 


\section{Sumário}

$\begin{array}{ll}\text { Introdução } & 15\end{array}$

1 Introdução às Equações Diferenciais Funcionais 19

1.1 Notações e definições . . . . . . . . . . . . . . . . . . . . . . . 19

1.2 Teoria qualitativa de EDFs . . . . . . . . . . . . . . . . . . 22

1.3 Propriedades do operador solução . . . . . . . . . . . . . . . . . . . . . . . . . . . 23

1.4 Sistemas lineares gerais . . . . . . . . . . . . . . . . . . . . . . . . . . . . 25

1.5 Teoria de Floquet para EDFs . . . . . . . . . . . . . . 27

2 Teoria de perturbação para semigrupos duais 31

2.1 Espaços sol-estrela $(\odot *)$. . . . . . . . . . . . . . . . 32

2.2 O problema protótipo . . . . . . . . . . . . . 34

2.3 EDFRs lineares como perturbações limitadas . . . . . . . . . . 41

2.3.1 Perturbações limitadas no caso $\odot$-reflexivo . . . . . . . 42

2.3.2 Perturbações limitadas com imagem de dimensão finita 45

3 Equações Diferenciais Ordinárias Generalizadas $\quad 51$

3.1 A Integral de Kurzweil . . . . . . . . . . . . . . . . . . . 51

3.2 Noções básicas de EDOs generalizadas . . . . . . . . . . . . . 57

3.3 Existência e unicidade de soluções . . . . . . . . . . . . . . . . . 60

3.4 Dependência contínua para EDOGs . . . . . . . . . . . . 61

4 Sistemas Periódicos Neutros $\quad \mathbf{6 5}$

4.1 Teoria espectral para sistemas neutros . . . . . . . . . . 65

4.2 Teoria espectral para sistemas periódicos neutros . . . . . . . 68

4.3 Uma classe de sistemas periódicos neutros . . . . . . . . . . 70

4.4 Comportamento assintótico . . . . . . . . . . . . . 77

4.5 Casos estudados . . . . . . . . . . . . . . 80 
4.5.1 Uma classe simples de EDFR periódicas . . . . . . . 80

4.5.2 Uma classe de EDFN com parte neutra periódica . . . 82

5 Existência de soluções limitadas para EDFRs não homogêneas $\mathbf{8 5}$

5.1 Introdução . . . . . . . . . . . . . . . . . . . 85

5.2 Sistemas de evolução e perturbações limitadas . . . . . . . . . 86

5.3 EDFRs lineares não homogêneas . . . . . . . . . . . . . . . . . 89

5.4 Soluções limitadas para EDFRs não homogêneas . . . . . . . . 94

6 EDFNs em medida e EDOs generalizadas 99

6.1 Introdução . . . . . . . . . . . . . . . . . . . . . . . . . 99

6.2 Correspondência entre EDFNs em medida e EDOs generalizadas101

6.3 Existência e unicidade de soluções . . . . . . . . . . . . . . . 117

6.4 Dependência contínua em relação aos parâmetros . . . . . 119

6.5 Um exemplo . . . . . . . . . . . . . . . . . . . . . . 122

A Introdução à teoria de semigrupos fortemente contínuos e seus adjuntos

A.1 Definições . . . . . . . . . . . . . . . . . . 127

A.2 Espaço dual e Semigrupos adjuntos . . . . . . . . . . . 129

A.3 A integral fraca-* . . . . . . . . . . . . . . . 131

A.4 Variantes da fórmula da variação das constantes . . . . . . . 134

$\begin{array}{ll}\text { Referências } & 141\end{array}$

$\begin{array}{ll}\text { Índice Remissivo } & 149\end{array}$ 


\section{Introdução}

As equações diferenciais funcionais (EDFs) foram consideradas por primeira vez por Euler, Bernoulli, Lagrange, Laplace, Poisson entre outros matemáticos do século XIX. Neste período essas equações foram raramente estudadas. Contudo, a situação mudaria radicalmente na década dos anos 30 do século passado, devido a que muitos modelos que descrevem fenômenos mecânicos, físicos, biológicos, ecológicos entre outros, demandariam tomar em conta retardamentos existentes. Em outras palavras, em muitos sistemas o futuro dependeria não somente do presente, senão também do passado. Os primeiros problemas deste tipo foram considerados por Volterra (viscoelasticidade, 1909 [86]; modelos predador-presa, 1928-1931 [87,88]), Callendar \& Stevenson (instabilidade em sistemas com restardamento, 1936 [8]), Sievert (reação de células à radiação de raios-x, 1941 [81]), Andronov \& Mayer (retardamentos em sistemas de retroalimentação, 1946 [3]) e outros autores mais, que podem ser encontrados por exemplo na extensa bibliografia do livro de Kolmanovskii \& Myshkis [52].

Uma importante contribuição para o desenvolvimento da teoria das EDFs, tiveram os trabalhos de Myshkis, quem formulou por primeira vez o problema de valor inicial para as EDFs. Além disso, em seu livro [65], introduz uma classe geral de equações diferenciais funcionais com retardamento (EDFRs) e estudou algumas propriedades básicas das soluções. Cabe mencionar também o livro de Bellman \& Cooke [5], que mostra de maneira sistemática propriedades de tais equações.

Outra classe especial de equações a ser distinguida entre as EDFs, são as equações diferenciais funcionais neutras (EDFNs). Esta classe constitui uma generalização para as EDFRs, cujo estudo foi tornando-se independente devido ao requerimento de métodos mais refinados aos usados na teoria das EDFRs. Além do interesse teórico, temos a importância nas aplicações visto que frequentemente são usadas para o estudo de redes distribuídas contendo 
linhas de transmissão sem perdas, sistemas de realimentação para turbinas hidráulicas, entre outras aplicações (vide $[52,55]$ ).

Uma das propriedades de maior interesse nas últimas décadas é o comportamento assintótico das soluções de equações lineares com retardamento ou equações diferenciais lineares neutras, motivados pelos trabalhos pioneiros de Driver [26] e Driver, Sasser e Slater [27]. Alguns anos depois, Hale \& Verduyn Lunel [45, Seç. 8.3] mostraram uma aplicação da teoria de Floquet para equações diferenciais com retardamento no caso em que os retardamentos são múltiplos inteiros do período comum dos coeficientes periódicos. Frasson \& Verduyn Lunel [42] estudaram a mesma equação como uma aplicação de seus resultados. Alguns resultados mais recentes têm sido dados por Philos et al. em [53,68,69]. O artigo de Philos \& Purnaras [69] trata do comportamento assintótico de soluções para uma equação diferencial linear neutra de primeira ordem com coeficientes periódicos e retardos constantes, que são múltiplos do período comum. Lillo [59] considera a distribuição assintótica de multiplicadores exponenciais para equações diferença periódicas para o caso em que os retardos e o período estão racionalmente relacionados. Esses trabalhos inspiraram uma forma de equação em que estamos interessados, isto é, nosso primeiro resultado dado no Capítulo 4, será estabelecido para uma EDFN com coeficientes periódicos onde, a diferença dos trabalhos citados anteriormente, o período será múltiplo racional do retardamento. O método empregado estará baseado na análise das propriedades espectrais do operador monodrômico. Mostraremos que o comportamento assintótico será obtido desde que se conheça que um multiplicador característico do operador monodrômico seja dominante. Cabe mencionar que os resultados deste capítulo generalizam alguns resultados de Frasson \& Lunel [42] e estão contidos no artigo submetido [43].

Por outro parte, uma das ferramentas importantes para descrever o comportamento assintótico das EDFRs não autônomas é o conceito de dicotomia exponencial, que foi introduzido por Perron [67] num cenário de dimensão finita. Nesses últimos cinquenta anos, uma extensa literatura sobre este tópico foi forjada, tanto no caso de dimensão finita, como no caso geral estendendo o problema a considerar espaços de Banach de dimensão infinita, surgindo assim a preocupação de estabelecer condições necessárias e suficientes para que a dicotomia exponencial aconteça. Podemos citar por exemplo os trabalhos de Massera \& Schäffer 1958, 1966 [60,61]; Coopel, 1965-1978 [10-14]; Cooffman, 1967 [15]; Daleckii \& Krel̆n, 1974 [17]; Preda, 1985 [70]; Sacker \& Sell, 1974-1994 [75-78]; Rodrigues \& Ruas-Filho 1995 [71]; Latushkin, Randolph 
\& Schaubelt 1998 [58]; Sasu, 2006 [80], entre outros autores. No Capítulo 5 deste trabalho, faremos uso desta importante ferramenta, juntamente com a teoria de semigrupos duais sobre espaços de Banach não reflexivos (vide os artigos de Diekmann et al [19-23,25]), primeiramente para mostrar a correspondência entre as soluções de uma equação integral abstrata, derivada de uma versão diferente da fórmula da variação das constantes, e as soluções de uma EDFR não autônoma, não homogênea. Mediante essa correspondência estabeleceremos o teorema que garante a existência de soluções limitadas de EDFRs lineares não autônomas e não homogêneas, sempre que o termo forçante seja limitado, se o operador solução da EDFR homogênea associada gozar da propriedade da dicotomia exponencial.

Finalmente, em virtude do crescimento considerável do estudo das equações diferenciais ordinárias generalizadas (EDOs generalizadas), introduzidas por Kurzweil em [56] com o propósito de generalizar certos resultados de dependência contínua de EDOs em relação aos parâmetros, introduziremos neste trabalho uma classe geral de equações diferenciais funcionais neutras em medida, inspirados pelos trabalhos de Federson et al [2,28,30,32-34,37], onde mostram que as EDOs generalizadas englobam diversos tipos de equações diferenciais tais como EDFRs, equações diferenciais funcionais com impulsos (EDFIs), equações diferenciais em medida (EDMs), etc. Assim, no Capítulo 6, será mostrado que as EDFNs em medida podem ser relacionadas com uma classe de EDOs generalizadas, obtendo resultados de existência e unicidade das soluções, dependência contínua em relação aos parâmetros, através da dita correspondência entre as equações. Exibiremos, também, um exemplo que ilustra a correspondência entre estas equações diferenciais. Mencionamos que os resultados deste capítulo estão contidos no artigo [31]. Referências básicas para o estudo de EDOs generalizadas podem ser encontradas nos trabalhos de Kurzweil $[56,57]$ e no livro de Schwabik [74].

Dividiremos este trabalho em seis capítulos, de forma que os três primeiros capítulos estarão dedicados à apresentação dos preliminares necessários para o entendimento dos resultados inéditos contidos nos últimos três capítulos, já introduzidos acima.

No capítulo 1, apresentaremos brevemente a teoria básica das equações diferenciais funcionais, colocando ênfase no estudo das propriedades da classe das EDFNs. Além disso, apresentaremos a teoria das EDFRs lineares periódicas com forma bastante geral, como encontrado no livro de Hale \& Lunel [45]. No capítulo 2, apresentaremos a teoria de perturbação para semigrupos duais, com a finalidade de mostrar que as EDFRs lineares podem ser vistas 
como uma perturbação limitada de uma EDFR trivial, à que chamaremos de problema protótipo. Estes resultados serão importantes no estabelecimento da fórmula da variação das constantes para os diversos tipos de equações dos capítulos seguintes, como EDFRs lineares não autônomes e depois também não homogêneas. No capítulo 3, descreveremos à teoria básica de EDOs generalizadas, necessárias para obter nossos resultados do Capítulo 6. Concluiremos este trabalho com o Apêndice A, onde apresentamos fatos conhecidos da teoria de $\mathcal{C}_{0}$-semigrupos e semigrupos duais. Além disso, para o bom entendimento do Capítulo 2, daremos a definição de integral fraca-*, exibindo alguns resultados detalhadamente. Para finalizar o apêndice, apresentaremos algumas variantes da fórmula da variação das constantes no contexto da teoria de perturbação de semigrupos duais. 


\section{Capítulo 1}

\section{Introdução às Equações Diferenciais Funcionais}

Neste capítulo, introduziremos a teoria básica de uma classe geral de Equações Diferenciais Funcionais (EDFs). Resultados de existência, unicidade, continuação e dependência contínua das soluções das EDFs serão apresentados na segunda seção. Uma vez garantida a existência e unicidade das soluções definiremos o operador solução cujas propriedades serão estudadas na terceira seção. Para finalizar este capítulo, nas duas últimas seções trataremos das EDFs lineares, prestando uma atenção especial aos sistemas periódicos.

Este capítulo, é um resumo dos resultados apresentados no livro de Hale \& Verduyn Lunel [45, Capítulos II, III, VIII e IX].

\subsection{Notações e definições}

Consideremos uma função $x$ definida sobre algum intervalo do eixo real, a valores em $\mathbb{C}^{n}$. Na teoria das EDFs, estamos interessados em situações onde a variável no eixo real é interpretada como o tempo, e portanto será denotada por $t$, e onde a derivada de $x$ em algum tempo $t$, depende linearmente de valores de $x$ para tempos menores ou iguais a $t$. Mais precisamente, pediremos a existência de $h>0$ tal que $\dot{x}(t)$ dependerá linearmente de $x(\tau)$ para $\tau \in$ $[t-h, t]$.

Seja $\mathcal{C}=C\left([-h, 0], \mathbb{C}^{n}\right)$ o espaço de Banach das funções contínuas de $[-h, 0]$ em $\mathbb{C}^{n}$, munida com a norma do supremo. Além disso, denotaremos 
ao longo deste trabalho a função memória $x_{t} \in \mathcal{C}$ definida por

$$
x_{t}(\theta)=x(t+\theta), \quad-h \leqslant \theta \leqslant 0
$$

Se $L$ é uma aplicação linear limitada de $\mathcal{C}$ em $\mathbb{C}^{n}$, segue do Teorema da Representação de Riesz (vide [72,73]), que $L$ pode ser representada por

$$
L \varphi=\int_{0}^{h} d \zeta(\theta) \varphi(-\theta)
$$

onde $\zeta$ é uma função de variação limitada em $[0, h]$, normalizada, tal que $\zeta(0)=0$ e $\zeta$ é contínua a direita em $(0, h)$ com valores no espaço das matrizes $\mathbb{C}^{n \times n}$. Este conjunto será denotado por $\operatorname{NBV}\left([0, h], \mathbb{C}^{n \times n}\right)$. Podemos estender trivialmente $\zeta \in \operatorname{NBV}([0, h], \cdot)$ em $\mathbb{R}$ por $\zeta(\theta)=0$ se $\theta<0$ e $\zeta(\theta)=\zeta(h)$ se $\theta>r$.

Observação 1.1. Em (1.2) escrevemos $d \zeta$ na frente de $\varphi$ para indicar que $\zeta$ é uma matriz $n \times n$ e $\varphi$ é um $n$-vetor coluna, portanto a integral é um vetor coluna.

Para definir uma classe geral de equações diferenciais funcionais, precisamos das seguintes definições.

Definição 1.2. Seja $\Lambda$ um subconjunto de um espaço métrico. Sejam $t \in \Lambda$ e $L(t): \mathcal{C} \rightarrow \mathbb{C}^{n}$ uma aplicação linear limitada tal que

$$
L(t) \varphi=\int_{0}^{h} d_{\theta}[\zeta(t, \theta)] \varphi(-\theta) .
$$

Se $\beta \in \mathbb{R}$ e a matriz $A(t ; \beta, L)=\zeta\left(t, \beta^{+}\right)-\zeta\left(t, \beta^{-}\right)$é não singular em $t=t_{0}$, dizemos que $L(t)$ é atômica em $\beta$ em $t_{0}$. Se $A(t ; \beta, L)$ é não singular no conjunto $K \subseteq \Lambda$, dizemos que $L(t)$ é atômica em $\beta$ sobre $K$.

Assim a grosso modo, uma aplicação linear é atômica em $\beta$ quando há uma dependência explícita do valor de $\varphi(\beta)$.

Definição 1.3 (Vide [45, Seç. II.5]). Seja $\Omega \subset \mathbb{R} \times \mathcal{C}$ um subconjunto aberto com elementos $(t, \phi)$. Uma aplicação $N: \Omega \rightarrow \mathbb{C}^{n}$ é chamada atômica em $\beta$ sobre $\Omega$ se $N$ é contínua junto com sua primeira e segunda derivadas de Fréchet em relação a $\phi$; e $N_{\phi}$, a derivada em relação a $\phi$, é atômica em $\beta$ sobre $\Omega$. 
Agora podemos definir a classe de equações diferenciais funcionais neutras (EDFNs).

Definição 1.4 (Vide [45, Seç. II.7]). Sejam $\Omega \subset \mathbb{R} \times \mathcal{C}$ aberto e $f, N: \Omega \rightarrow \mathbb{C}^{n}$ aplicações contínuas com $N$ atômica em zero. A seguinte relação

$$
\frac{d}{d t} N\left(t, x_{t}\right)=f\left(t, x_{t}\right), \quad t \geqslant 0
$$

define a $\operatorname{EDFN}(N, f)$. A função $N$ será chamada de operador diferença da $\operatorname{EDFN}(N, f)$.

Definição 1.5 (Vide [45, Seç. II.7]). Uma função $x$ é chamada solução $d a$ $\operatorname{EDFN}(N, f)$ se existem $\sigma \in \mathbb{R}, A>0$, tais que $x \in C\left([\sigma-h, \sigma+A), \mathbb{C}^{n}\right)$, $\left(t, x_{t}\right) \in \Omega, t \in[\sigma, \sigma+A), N\left(t, x_{t}\right)$ é continuamente diferenciável e satisfaz a equação (1.3) em $[\sigma, \sigma+A)$. Para $\sigma \in \mathbb{R}, \varphi \in \mathcal{C}$ dados, e $(\sigma, \varphi) \in \Omega$, denotamos $x(\sigma, \varphi)=x(\cdot ; \sigma, \varphi)$ como a solução de (1.3) com condição inicial $x_{\sigma}=\varphi$ ou simplesmente uma solução através de $(\sigma, \varphi)$.

Observação 1.6. Se $N(t, \phi)=N_{0}(t) \phi-g(t), f(t, \phi)=L(t) \phi+h(t)$ onde $N_{0}(t)$ e $L(t)$ são aplicações lineares em $\phi$, então a $\operatorname{EDFN}(\mathrm{N}, \mathrm{f})$ é chamada linear. $\mathrm{A} \operatorname{EDFN}(\mathrm{N}, \mathrm{f})$ é linear homogênea se $g \equiv 0$ e $h \equiv 0$ e linear não homogênea, se $g \not \equiv 0$ ou $h \not \equiv 0$. Se $N(t, \phi)$ e $f(t, \phi)$ não dependem de $t$, a $\operatorname{EDFN}(\mathrm{N}, \mathrm{f})$ é chamada autônoma.

Exemplo 1.7. Se $N \phi=\phi(0)$, então $N$ é atômica em zero e para qualquer função $f: \Omega \rightarrow \mathbb{C}^{n},(N, f)$ define uma EDFN da forma

$$
\frac{d}{d t} x(t)=f\left(t, x_{t}\right)
$$

que é conhecida como equação diferencial funcional com retardamento (EDFR).

Exemplo 1.8. Se $h>0, B$ é uma matriz constante $n \times n$, então $N \phi=$ $\phi(0)-B \phi(-h)$ é atômica em zero. Assim, $(N, f) \operatorname{com} f: \Omega \rightarrow \mathbb{C}^{n}$ é contínua, define uma EDFN da forma

$$
\frac{d}{d t}[x(t)-B x(t-h)]=f\left(t, x_{t}\right)
$$

Exemplo 1.9. Se $h>0, a, b, c$ escalares, $N \phi=\phi(0)-a \phi^{2}(-h)$, então

$$
\frac{d}{d t}\left(x(t)-a x^{2}(t-h)\right)=b x(t)+c x(t-h)
$$

é uma EDFN. 
Exemplo 1.10. Sejam $h_{j} \in[0, h], j=1, \ldots, N$, números reais, $g: \mathbb{R}^{N} \rightarrow \mathbb{R}^{n}$ uma função contínua. Se $N \phi=\phi(0)-g\left(\phi\left(-h_{1}\right), \ldots, \phi\left(-h_{N}\right)\right)$, então a equação diferença

$$
\frac{d}{d t}\left[x(t)-g\left(x\left(t-h_{1}\right), \ldots, x\left(t-h_{N}\right)\right)\right]=0
$$

define uma EDFN.

Portanto, exemplos de EDFNs são equações diferenciais funcionais com retardamento, assim como equações diferença; para maiores detalhes sugerimos ao leitor consultar o livro de Hale \& Verduyn Lunel [45, Seção II.7].

\subsection{Teoria qualitativa de EDFs}

A seguir enunciaremos os teoremas básicos sobre existência, unicidade e dependência contínua com relação às condições iniciais. As demonstrações destes resultados podem ser encontradas em [45, Capítulo 2].

O seguinte teorema trata sobre a existência e unicidade de soluções da $\operatorname{EDFN}(1.3)$.

Teorema 1.11 (Vide [45, Teorema II.8.1 e Teorema II.8.3]). Se $\Omega$ é um aberto de $\mathbb{R} \times \mathcal{C}$ e $(\sigma, \varphi) \in \Omega$, então existe uma solução da $\operatorname{EDFN}(N, f)$ que passa por $(\sigma, \varphi)$. Além disso, se $f: \Omega \rightarrow \mathbb{C}^{n}$ for lipschitziana na segunda variável em subconjuntos compactos de $\Omega$, então, para qualquer $(\sigma, \varphi) \in \Omega$, existe uma única solução da $\operatorname{EDFN}(N, f)$ que passa por $(\sigma, \varphi)$.

O próximo teorema exibe um resultado de dependência contínua.

Teorema 1.12 (Vide [45, Teorema II.8.2]). Sejam $\Omega \subset \mathbb{R} \times \mathcal{C}$ aberto, $\Lambda$ um subespaço de um espaço de Banach, e $N: \Omega \times \Lambda \rightarrow \mathbb{C}^{n}, f: \Omega \times \Lambda \rightarrow \mathbb{C}^{n}$ satisfazendo as seguintes hipóteses:

1. $N(t, \varphi, \lambda)$ é atômico em zero para cada $(t, \varphi) \in \Omega$ uniformemente com respeito $a \lambda$.

2. $N(t, \varphi, \lambda)$ e $f(t, \varphi, \lambda)$ são contínuas em $(t, \varphi) \in \Omega$ para cada $\lambda \in \Lambda$ e também contínuas em $\left(t, \varphi, \lambda_{0}\right)$ para $(t, \varphi) \in \Omega$.

3. $A \operatorname{EDFN}\left(N\left(\cdot, \lambda_{0}\right), f\left(\cdot, \lambda_{0}\right)\right)$ tem uma única solução definida num intervalo $[\sigma-r, b)$ que passa por $(\sigma, \varphi) \in \Omega$. 
Então existe uma vizinhança $V=V\left(\sigma, \varphi, \lambda_{0}\right)$ de $\left(\sigma, \varphi, \lambda_{0}\right)$ tal que para qualquer $\left(\sigma^{\prime}, \varphi^{\prime}, \lambda^{\prime}\right) \in V$, todas as soluções $x\left(\sigma^{\prime}, \varphi^{\prime}, \lambda^{\prime}\right)$ da $\operatorname{EDFN}\left(N\left(\cdot, \lambda^{\prime}\right), f\left(\cdot, \lambda^{\prime}\right)\right)$ que passam por $\left(\sigma^{\prime}, \varphi^{\prime}\right)$ existem em $\left[\sigma^{\prime}-r, b\right]$ e $x_{t}\left(\sigma^{\prime}, \varphi^{\prime}, \lambda^{\prime}\right)$ é contínua em $\left(t ; \sigma^{\prime}, \varphi^{\prime}, \lambda_{0}\right)$ para $t \in[\sigma, \sigma+A] e\left[\sigma^{\prime}, \varphi^{\prime}, \lambda^{\prime}\right] \in V\left(\sigma, \varphi, \lambda_{0}\right)$.

Definição 1.13. Seja $x$ uma uma solução da EDFN (1.3), definida no intervalo $[\sigma, a), \sigma<a$ dizemos que $\hat{x}$ é continuação de $x$ se:

1. existe $b>a$ tal que $\hat{x}$ é definido em $[\sigma-r, b)$,

2. $\hat{x}$ coincide $\operatorname{com} x$ em $[\sigma-r, a)$, e

3. $\hat{x}$ satisfaz a $\operatorname{EDFN}(1.3)$ em $[\sigma, b)$.

Uma solução $x$ é não-continuável se tal continuação não existe. Neste caso, o intervalo $[\sigma, a)$ é denominado intervalo maximal de existência da solução de $x$. A existência de uma solução não-continuável segue do Lema de Zorn. Além disso, o intervalo maximal de existência é aberto.

Para finalizar esta seção, enunciaremos o resultado que garante a existência do intervalo maximal.

Teorema 1.14 (Vide [45, Teorema II.8.4]). Considere uma $\operatorname{EDFN}(f, N)$ com $\Omega \subset \mathbb{R} \times \mathcal{C}$ aberto, $W \subset \Omega$ fechado e limitado, e suponha que exista uma $\delta$-vizinhança de $W$ em $\Omega$. Se $f$ mapeia $W$ em um conjunto limitado de $\mathbb{C}^{n}$, $N(t, \varphi)$ e $N_{\varphi}(t, \varphi)$ (derivada em relação a $\varphi$ ) são uniformemente contínuas em $W, N$ é uniformemente atômico em $0 \mathrm{em} W$, e $x$ é uma solução nãocontinuável da $\operatorname{EDFN}(N, f)$ em $[\sigma-r, b)$, então existe um $t^{\prime} \in[a, b)$ tal que $\left(t^{\prime}, x_{t^{\prime}}\right) \notin W$.

\subsection{Propriedades do operador solução}

Consideremos um sistema autônomo, seja $T(t)$ o operador que a cada tempo $t$ atribui o estado $x_{t}$. Então,

1. $T(0)=I$ (o operador identidade),

2. $T(t+s)=T(t) T(s), \quad t, s \geqslant 0$.

onde a segunda propriedade deriva da unicidade de soluções. Estas propriedades são sintetizadas dizendo que a família $\{T(t)\}_{t \geqslant 0}$ constitui um semigrupo 
Introdução às Equações Diferenciais Funcionais

de operadores a um parâmetro, o adjetivo "semi" expressa que a solução para $t<0$ não necessariamente existe ou, em outras palavras, que $t$ está restrito a valores não negativos.

Na sequência, descreveremos as propriedades deste operador para a classe geral das EDFNs. Uma descrição mais detalhada pode ser encontrada no livro de Hale \& Verduyn Lunel [45, Seção III.7].

Considere uma $\operatorname{EDFN}(N, f)$ e suponha que as condições do Teorema 1.11 para a unicidade de soluções estejam satisfeitas para cada $(\sigma, \varphi) \in \Omega$. Então existe um $t_{\sigma, \varphi}$ e uma função $x$ que é uma solução não-continuável da $\operatorname{EDFN}(N, f)$ em $\left[\sigma-r, t_{\sigma, \varphi}\right)$ que passa por $(\sigma, \varphi)$. Seja $\Omega_{\sigma} \subset \mathcal{C}$ definido por $\Omega_{\sigma}=\{\varphi \in \mathcal{C}:(\sigma, \varphi) \in \Omega\}$. Se trabalharmos somente com soluções nãocontinuáveis, podemos definir um operador $T(t, \sigma): \Omega_{\sigma} \rightarrow \mathcal{C}$ tal que para cada $\varphi \in \Omega_{\sigma}$, e $t \in\left[\sigma, t_{\sigma, \varphi}\right)$

$$
T(t, \sigma) \varphi=x_{t}(\sigma, \varphi) .
$$

Este operador será dito operador solução da $\operatorname{EDFN}(N, f)$.

Para as EDFRs, o operador solução $T(t, \sigma)$ aumenta a diferenciabilidade. Além disso, se uma solução da EDFR pode ser estendida em direção negativa passando por $(\sigma, \phi)$, então $\phi$ deve satisfazer algumas condições de diferenciabilidade. Não obstante, para a classe de EDFNs, nenhuma destas propriedades valem.

Como veremos, podemos escrever o operador solução de uma EDFN como perturbação, por meio de um operador compacto, de um outro operador solução. Restringimos nossa atenção para o caso autônomo para evitar notações excessivas.

Consideremos a Equação Diferença (ED)

$$
N y_{t}=0, \quad t \geqslant 0
$$

onde $N$ é linear, contínuo e atômico em 0 . Se $\mathcal{C}_{N}=\{\varphi \in \mathcal{C}: N \varphi=0\}$, então a equação diferença (1.6) define um semigrupo de operadores lineares $T_{N}(t)$, $t \geqslant 0$, em $\mathcal{C}_{N}$, onde $T_{N}(t) \psi=y_{t}(\psi)$ com $y_{t}(\psi)$ sendo a solução de (1.6) que passa por $(0, \psi)$.

Seja $f: \mathcal{C} \rightarrow \mathbb{C}^{n}$ uma função contínua e $T_{N, f}(t)$ o semigrupo definido pela $\operatorname{EDFN}(N, f)$,

$$
\frac{d}{d t} N x_{t}=f\left(x_{t}\right), \quad t \geqslant 0 .
$$

Então, podemos enunciar um teorema para a representação do semigrupo dado em (1.5). 
Teorema 1.15 (Vide [45, Lema III.7.1 e Teorema III.7.3]). Sejam $N: \mathcal{C} \rightarrow \mathbb{R}^{n}$ linear, limitado e atômico em $0, \Phi=\left(\varphi_{1}, \ldots, \varphi_{n}\right)$ uma $n$-upla de funções tal que $N \Phi=I$, e $\Psi=I-\Phi N$. Se $f$ é completamente contínua e $T_{N, f}(t)$ é um operador limitado para cada $t>0$, então

$$
T_{N, f}(t)=T_{N}(t) \Psi+U(t)
$$

onde $U(t): \mathcal{C} \rightarrow \mathcal{C}, t \geqslant 0$, é compacto.

\subsection{Sistemas lineares gerais}

Nesta seção, estamos interessados no problema de valor inicial (PVI) para equações diferenciais funcionais lineares não autônomas, dada pela seguinte relação

$$
\left\{\begin{array}{l}
\frac{d}{d t} N(t) x_{t}=L(t) x_{t}, \quad t \geqslant 0 \\
x_{0}=\varphi, \quad \varphi \in \mathcal{C}
\end{array}\right.
$$

em que $N(t), L(t): \mathcal{C} \rightarrow \mathbb{C}^{n}$ são aplicações lineares contínuas dadas por

$$
L(t) \varphi=\int_{0}^{h} d[\zeta(t, \theta)] \varphi(-\theta), \quad N(t) \varphi=\varphi(0)-\int_{0}^{h} d[\mu(t, \theta)] \varphi(-\theta) .
$$

onde $\zeta(t, \cdot), \mu(t, \cdot) \in \mathrm{NBV}$ e $\mu$ tem que ser uniformemente não-atômica em 0 , como veremos abaixo.

As funções $\mu$ e $\zeta$ são chamadas núcleos e satisfazem as seguintes hipóteses.

(H1) O núcleo $\zeta: \mathbb{R} \times \mathbb{R} \rightarrow \mathbb{C}^{n \times n},(t, \theta) \mapsto \zeta(t, \theta)$, é mensurável em $(t, \theta)$, normalizado de forma que

$$
\zeta(t, \theta)=0, \quad \theta \leqslant 0 ; \quad \zeta(t, \theta)=\zeta(t, h), \quad \theta \geqslant h
$$

$(\mathrm{H} 2) \zeta(t, \theta)$ é contínua à direita em $\theta$ sobre o intervalo $(0, h)$ e tem variação limitada em $\theta$ sobre $[0, h]$, para cada $t$.

(H3) Existe uma função $m \in L_{\text {loc }}^{1}(\mathbb{R}, \mathbb{R})$ tal que

$$
\operatorname{Var}_{[0, h]} \zeta(t, \cdot) \leqslant m(t)
$$


(H4) O núcleo $\mu: \mathbb{R} \times \mathbb{R} \rightarrow \mathbb{C}^{n \times n},(t, \theta) \mapsto \mu(t, \theta)$, é mensurável em $(t, \theta)$, normalizado de forma que

$$
\mu(t, \theta)=0, \quad \theta \leqslant 0 ; \quad \mu(t, \theta)=\mu(t, h), \quad \theta \geqslant h .
$$

(H5) $\mu(t, \theta)$ é contínua à direita em $\theta$ sobre o intervalo $(0, h)$ e tem variação limitada em $\theta$ sobre $[0, h]$ uniformemente em $t$ e tal que $t \mapsto N(t) \phi$ é contínua para cada $\phi$.

(H6) $\mu$ é uniformemente não-atômica em zero, isto é, para $\epsilon>0$ existe $\delta>0$ tal que

$$
\operatorname{Var}_{[0, \delta]} \mu(t, \cdot)<\epsilon, \quad \forall t \in \mathbb{R} .
$$

Exemplo 1.16. Se a aplicação linear $N(t)$ é tal que $N(t) \varphi=\varphi(0)$, então a equação (1.7) representa uma classe importante de equações, chamada equações diferenciais funcionais lineares com retardamento,

$$
\left\{\begin{array}{l}
\frac{d}{d t} x(t)=L(t) x_{t}, \quad t \geqslant 0, \\
x_{0}=\varphi, \quad \varphi \in \mathcal{C}
\end{array}\right.
$$

ou alternativamente, usando a representação para $L(t)$ dado pela Equação (1.8), podemos reescrever (1.9) como

$$
\left\{\begin{array}{l}
\dot{x}(t)=\int_{0}^{h} d \zeta(t, \theta) \varphi(-\theta), \quad t \geqslant 0 \\
x_{0}=\varphi, \quad \varphi \in \mathcal{C}
\end{array}\right.
$$

Um extenso estudo foi desenvolvido nas últimas décadas em quanto ao tratamento destas equações, veja por exemplo [24,45] para uma introdução compreensiva.

Exemplo 1.17. Consideremos a seguinte EDFN linear

$$
\frac{d}{d t}[x(t)+C x(t-1)]=A x(t)+B x(t-1), \quad t \geqslant 0
$$

onde $A, B$ e $C$ são matrizes $n \times n$, e pode ser reescrita na forma (1.7) no caso autônomo tomando $h=1, \mu(\theta)=0$ se $\theta<1, \mu(\theta)=-C$ para $\theta \geqslant 1$ (isto é, o "salto" é de $-C$ em $\theta=1$, e $\zeta(\theta)=0$ para $\theta \leqslant 0, \zeta(\theta)=A$ para $0<\theta<1$ e $\zeta(\theta)=A+B$ para $\theta \geqslant 1$ (a variação de $\zeta$ em (1.8) está concentrada em $\theta=0$ e $\theta=1$, onde os saltos são $A$ e $B$ respectivamente). 
Os resultados de existência e unicidade para soluções também se aplicam para as EDFNs lineares, como no caso geral. Em particular podemos citar o seguinte teorema.

Teorema 1.18 (Vide [45, Teorema IX.1.1]). Suponha que as condições (H1)(H6) sobre $\zeta$ e $\mu$ estejam satisfeitas. Para qualquer $\sigma \in \mathbb{R}, \varphi \in \mathcal{C}$, existe uma única função $x(\cdot ; \sigma, \varphi)$ contínua em $[\sigma-h, \infty)$ que satisfaz o sistema (1.7).

\subsection{Teoria de Floquet para EDFs}

O propósito desta seção é apresentar a teoria geral para sistemas periódicos lineares com retardamento de forma análoga à teoria de Floquet para equações diferenciais ordinárias. Faremos um resumo do Capítulo 8 do livro de Hale \& Verduyn Lunel [45] e do artigo de Stokes [84].

Seja $L(t): \mathcal{C} \rightarrow \mathbb{C}^{n}$ uma aplicação linear que satisfaz as condições $(H 1)$ (H3) da Seção 1.4 e suponha que exista $\omega>0$ tal que $L(t+\omega)=L(t)$ para todo $t$.

Consideremos o sistema

$$
\dot{x}(t)=L(t) x_{t} .
$$

É conhecido que para qualquer $s \in \mathbb{R}, \varphi \in \mathcal{C}$, existe uma solução $x=x(s, \varphi)$ da Equação (1.12) definida em $[s, \infty)$ e $x_{t}(s, \varphi)$ é uma função contínua em $t$, $s$ e $\varphi$. Definimos o operador $T(t, s): \mathcal{C} \rightarrow \mathcal{C} \operatorname{como} T(t, s) \varphi=x_{t}(s, \varphi)$ para todo $t \geqslant s$ e $\varphi \in \mathcal{C}$. A periodicidade da equação (1.12) implica que

$$
T(t+\omega, s+\omega)=T(t, s), \quad t \geqslant s
$$

Isto com a propriedade de semigrupo $T(t, \tau) T(\tau, s)=T(t, s), s \leqslant \tau \leqslant t$, nos dá que

$$
T(t+\omega, s)=T(t, s) T(s+\omega, s) \text {, para todo } t \geqslant s .
$$

Definimos a aplicação monodrômica ou aplicação periódica $\Pi(s): \mathcal{C} \rightarrow \mathcal{C}$ associada a (1.12) como segue

$$
\Pi(s) \varphi=T(s+\omega, s) \varphi, \quad \varphi \in \mathcal{C} .
$$

$\Pi(s)$ não é completamente contínua, se $\omega<h$, no entanto para qualquer inteiro $m$ satisfazendo $m \omega \geqslant h, \Pi^{m}(s)$ é um operador completamente contínuo e aplicando o teorema espectral e a teoria para operadores compactos temos as seguintes afirmações para $\Pi(s)$. 
i. O espectro de $\Pi(s), \sigma(\Pi(s))$ é um conjunto compacto do plano complexo no máximo contável.

ii. $0 \in \sigma(\Pi(s))$ e se $\mu \in \sigma(\Pi(s)), \mu \neq 0, \mu$ pertence ao espectro pontual de $\Pi(s), \sigma_{p}(\Pi(s))$, isto é, $\Pi(s) \varphi=\mu \varphi$ tem uma solução não nula $\varphi \in \mathcal{C}$ Qualquer $\mu \neq 0$ em $\sigma_{p}(\Pi(s))$ é chamado de multiplicador característico ou multiplicador Floquet da equação (1.12) e $\lambda$ tal que $\mu=e^{\lambda \omega}$ é chamado expoente característico da equação (1.12).

iii. Dado $\mu \neq 0, \mu \in \sigma_{p}(\Pi(s))$, como $\Pi(s)$ é compacto, existem dois subespaços fechados $E_{\mu}(s), Q_{\mu}(s)$ de $\mathcal{C} \operatorname{com} E_{\mu}(s)$ de dimensão finita tais que

1. $\mathcal{C}=E_{\mu}(s) \oplus Q_{\mu}(s)$;

2. $\Pi(s) E_{\mu}(s) \subset E_{\mu}(s), \Pi(s) Q_{\mu}(s) \subset Q_{\mu}(s)$;

3. $\sigma\left(\left.\Pi(s)\right|_{E_{\mu}(s)}\right)=\{\mu\}$ e $\sigma\left(\left.\Pi(s)\right|_{Q_{\mu}(s)}\right)=\sigma(\Pi(s)) \backslash\{\mu\}$.

iv. A dimensão de $E_{\mu}(s), d_{\mu}=\operatorname{dim}\left(E_{\mu}(s)\right)$ é chamada multiplicidade do multiplicador característico $\mu$.

Chamaremos $E_{\mu}(s)$, o autoespaço de $\Pi(s)$ correspondente a $\mu$. Como em geral a dimensão de $E_{\mu}(s)$, não tem relação com a dimensão de $\mathbb{C}^{n}$, não podemos falar em termos de matriz fundamental como no caso das equações diferenciais ordinárias. No entanto, seja $\phi_{1}(s), \ldots, \phi_{d_{\mu}}(s)$ uma base para $E_{\mu}(s)$, e denote por $\Phi$ o vetor linha $\Phi=\left\{\phi_{1}, \ldots, \phi_{d_{\mu}}\right\}$. Como $E_{\mu}(s)$ é invariante por $\Pi(s)$, existe uma matriz quadrada constante $M_{\mu}$ de ordem $d_{\mu}$ tal que

$$
\Pi(s) \Phi(s)=\Phi(s) M_{\mu}(s)
$$

e a propriedade (iii3) implica que o único autovalor de $M_{\mu}(s)$ é $\mu \neq 0$, portanto $M_{\mu}(s)$ é não singular, e $B=B_{s}=\frac{1}{\omega} \log M_{\mu}(s)$ está bem definido.

Defina o $d_{\mu}$-vetor $P(t)$ com elementos em $\mathcal{C}$ da seguinte forma

$$
P(t)=T(t, 0) \Phi e^{-B t}
$$

onde $\Phi=\Phi(0)$. Então para $t \geqslant 0, P(t)$ é $\omega$-periódica. Com efeito,

$$
\begin{aligned}
P(t+\omega) & =T(t+\omega) \Phi e^{-B(t+\omega)}=T(t, 0) T(\omega, 0) \Phi e^{-B(t+\omega)} \\
& =T(t, 0) \Pi(0) \Phi e^{-B \omega} e^{-B t}=T(t, 0) \Phi M_{\mu}(0) e^{-B \omega} e^{-B t}=T(t, 0) \Phi e^{-B t} \\
& =P(t) .
\end{aligned}
$$


Portanto,

$$
T(t, 0) \Phi=P(t) e^{B t}, t \geqslant 0 .
$$

Estendemos a definição de $P(t)$ para $t \in(-\infty, \infty)$ pela periodicidade de $P(t)$. Assim, temos que a função $x_{t}(0, \Phi)=T(t, 0) \Phi=P(t) e^{B t}$ esta bem definida para $t \in \mathbb{R}$ e cada coluna desta matriz é uma solução da equação (1.12). Isto completa a prova do seguinte lema.

Lema 1.19 (Vide [45, Lema VIII.1.2]). Se $\mu$ é multiplicador característico da equação (1.12) e $\Phi$ é uma base para $E_{\mu}$ de dimensão $d_{\mu}$, existe uma matriz $B$ de ordem $d_{\mu} \times d_{\mu}, \sigma\left(e^{B \omega}\right)=\{\mu\}$ e uma função matriz $P(t)$ de ordem $n \times d_{\mu}$ com cada coluna em $\mathcal{C}, P(t+\omega)=P(t), t \in(-\infty, \infty)$ tal que se $\phi=\Phi b$, então $x_{t}(\phi)$ esta definida para $t \in(-\infty, \infty)$ e $x_{t}(0, \phi)=P(t) e^{B t} b$, $t \in(-\infty, \infty)$.

Em particular, $\mu=e^{\lambda \omega}$ é um multiplicador característico da equação (1.12) se e somente se existe uma solução não nula da equação (1.12) da forma $x(t)=p(t) e^{\lambda t}$, onde $p(t+\omega)=p(t)$.

Seja $\phi \in E_{\mu}$. Como $x_{t}(0, \phi)(\theta)=x(0, \phi)(t+\theta)=x_{t+\theta}(0, \phi)(0)$, segue que $P(t)(\theta)=P(t+\theta)(0) e^{B \theta}$, para $\theta \in[-h, 0]$. Portanto se colocamos $\tilde{P}(t+\theta)=P(t+\theta)(0)$ temos $\Phi(\theta)=\tilde{P}(\theta) e^{B \theta} \mathrm{e}$

$$
x(0, \phi)(t)=\tilde{P}(t) e^{B t} b, \quad t \in(-\infty, \infty), \quad \phi=\Phi b
$$

Portanto, as soluções da equação (1.12) com condição inicial em $E_{\mu}(s)$ são de tipo Floquet, ou seja, se $\mu=e^{\lambda \omega}$, as soluções são da forma $e^{\lambda t}$ vezes um polinômio com coeficientes periódicos em t de período $\omega$.

Enquanto $E_{\mu}(s)$ em geral depende de $s$, é importante notar que $\sigma(\Pi(s))=$ $\sigma(\Pi(r))$ para quaisquer $r, s$ em $\mathbb{R}$. Com efeito, para $\mu \in \sigma(\Pi(s)) \backslash\{0\}$

$$
\begin{aligned}
\Pi(r) T(r, s) \Phi(s) & =T(r+\omega, r) T(r, s) \Phi(s)=T(r+\omega, s) \Phi(s) \\
& =T(r, s) T(s+\omega, s) \Phi(s)=T(r, s) \Pi(s) \Phi(s) \\
& =T(r, s) \Phi(s) M_{\mu}(s) .
\end{aligned}
$$

Portanto,

$$
\begin{aligned}
{[\mu I-\Pi(r)] T(r, s) \Phi(s) } & =\mu T(r, s) \Phi(s)-\Pi(r) T(r, s) \Phi(s) \\
& =\mu T(r, s) \Phi(s)-T(r, s) \Phi(s) M_{\mu}(s) \\
& =T(r, s) \Phi(s)\left[\mu I-M_{\mu}(s)\right]
\end{aligned}
$$


Logo $\mu \in \sigma(\Pi(r))$. Assim a dimensão de $E_{\mu}(r)$ é pelo menos tão grande quanto a dimensão de $E_{\mu}(s)$. E como podemos inverter $r$ e $s$ obtemos que $\sigma(\Pi(s))=\sigma(\Pi(r))$, isto é, os multiplicadores característicos de (1.12) são independentes do tempo inicial e se $\Phi(s)$ é uma base para $E_{\mu}(s)$, então $T(t, s) \Phi(s)$ é uma base para $E_{\mu}(t)$ para todo $t \in \mathbb{R}$. Em particular os subespaços $E_{\mu}(s)$ e $E_{\mu}(t)$ são difeomorfos para todo $s$ e $t$. 


\section{Capítulo 2}

\section{Teoria de perturbação para semigrupos duais}

Neste capítulo, consideraremos a equação diferencial funcional com retardamento linear autônoma

$$
\left\{\begin{array}{l}
\dot{x}(t)=L x_{t}, \quad t \geqslant 0 \\
x(\theta)=\varphi(\theta), \quad \theta \in[-h, 0]
\end{array}\right.
$$

com $\varphi \in X=C([-h, 0], \mathbb{C})$ e $L: X \rightarrow \mathbb{C}$ o operador linear dado, como no Teorema da Representação de Riesz, por

$$
L \varphi=\int_{0}^{h} d \zeta(\theta) \varphi(-\theta) .
$$

O operador solução $T(t): X \rightarrow X$ definido pela relação

$$
T(t) \varphi=x_{t}(\cdot, \varphi)
$$

é um $\mathcal{C}_{0}$-semigrupo, com gerador infinitesimal $A$ definido por

$$
\mathcal{D}(A)=\{\varphi \in X \mid \dot{\varphi} \in X, \dot{\varphi}(0)=L \varphi\}, \quad A \varphi=\dot{\varphi}
$$

Observemos que a EDF (2.1) está embutida no domínio de definição do gerador $A$. Portanto, mudar a regra que define a EDF implicará mudar o domínio do gerador infinitesimal e se quisermos relacionar soluções de várias equações mediante a fórmula da variação dos parâmetros, teremos muitas complicações técnicas. 
Para solucionar estes problemas no estudo das equações diferenciais funcionais com retardamento, faremos uso da teoria de dualidade de semigrupos. Assim, usaremos o símbolo $\odot$ (lê-se $s o l$ ) para definir o espaço $X^{\odot}$, como o subespaço invariante maximal onde o semigrupo dual $T^{*}(t)$ é fortemente contínuo. Tomando adjunto obtemos o espaço $X^{\odot *}$, no qual será mergulhado o espaço $X$. Neste espaço $X^{\odot *}$ teremos um semigrupo $T^{\odot *}(t)$.

O objetivo de introduzir a teoria $\odot *$ é fazer que o semigrupo $T(t)$ seja a restrição do semigrupo $T^{\odot *}(t)$, e conseguir a independência do domínio de $A$, da regra específica para a EDFR. De fato, a regra passa para a ação do gerador $A^{\odot *}$. Além disso o espaco $X^{\odot *}$ é essencial para a formulação precisa da fórmula da variação das constantes neste contexto. Uma descrição mais detalhada dos espaços $X^{\odot *}$ será dada na primeira seção.

Um outro objetivo deste capítulo é escrever uma EDFR qualquer como perturbação de uma EDFR trivial, que será chamada de problema protótipo cuja solução define um $\mathcal{C}_{0}$-semigrupo translação, porém o seu semigrupo dual não tem esta mesma propriedade. Na segunda seção, apresentaremos um estudo detalhado do problema protótipo, exibindo o espaço onde o semigrupo dual é fortemente contínuo. Será mostrado também a $\odot$-reflexividade do espaço $X$. Para finalizar este capítulo, na terceira seção, apresentaremos a teoria das perturbações limitadas no caso $\odot$-reflexivo assim como no caso em que a imagem seja dimensão finita. Daí, usando equações de renovação será possível escrever uma EDFR qualquer como perturbação do problema protótipo.

As principais referencias para este capítulo são os livros de Hille \& Phillips [50], Butzer \& Berens [7] e Diekmann et al [24, Capítulos II e III]

\subsection{Espaços sol-estrela $(\odot *)$}

Seja $\{T(t)\}_{t \geqslant 0}$ um semigrupo de operadores lineares limitados fortemente contínuos sobre um espaço de Banach $X$ e seja $A$ seu gerador infinitesimal. Para mais detalhes veja o Apêndice A.

Os operadores adjuntos $\left\{T^{*}(t)\right\}_{t \geqslant 0}$ formam um semigrupo fraco-* contínuo sobre o espaço dual $X^{*}$. Porém se munimos $X^{*}$ com a norma da topologia usual, $\left\{T^{*}(t)\right\}$ não necessariamente é fortemente contínuo (a menos que $X$ seja reflexivo), por exemplo veja a Observação 2.3 adiante. O operador $A^{*}$, o adjunto de $A$, é o gerador fraco-* de $\left\{T^{*}(t)\right\}$, não obstante não precisa ser densamente definido. 
No livro de Hille \& Phillips [50], mostraram a importância da teoria de dualidade de semigrupos para estabelecer a relação entre espaços e semigrupos.

Portanto, consideremos

$$
X^{\odot}=\left\{x^{*} \in X^{*} \mid \lim _{t \downarrow 0}\left\|T^{*}(t) x^{*}-x^{*}\right\|=0\right\}
$$

como o subespaço invariante maximal no qual $\left\{T^{*}(t)\right\}_{t \geqslant 0}$ é fortemente contínuo. $X^{\odot}$ é fechado com a norma e $X^{\odot}=\overline{\mathcal{D}\left(A^{*}\right)}$ (vide [7, Teorema 1.4.7]). Seja $\left\{T^{\odot}(t)\right\}_{t \geqslant 0}$ a restrição de $\left\{T^{*}(t)\right\}_{t \geqslant 0}$ a $X^{\odot}$ e temos que $\left\{T^{\odot}(t)\right\}_{t \geqslant 0}$ é semigrupo fortemente contínuo em $X^{\odot}$, e seja $A^{\odot}$ seu gerador infinitesimal. Temos que $A^{\odot}$ é parte de $A^{*}$ em $X^{\odot}$, isto é, a maior restrição de $A^{*}$ com domínio e imagem em $X^{\odot}$.

Repetindo os mesmos procedimentos obtemos um semigrupo fraco-* contínuo $\left\{T^{\odot *}(t)\right\}_{t \geqslant 0}$ em $X^{\odot *}$, o espaço dual de $X^{\odot}$, com gerador fraco-* $A^{\odot *}$. Então,

$$
X^{\odot \odot}=\left\{x^{\odot *} \in X^{\odot *} \mid \lim _{t \downarrow 0}\left\|T^{\odot *}(t) x^{\odot *}-x^{\odot *}\right\|=0\right\}
$$

é o subespaço invariante maximal no qual $\left\{T^{\odot *}(t)\right\}_{t \geqslant 0}$ é fortemente contínuo Uma interpretação simples da dualidade entre elementos de $X$ e $X^{\odot}$ fornece um mergulho natural $j$ de $X$ em $X^{\odot *}$,

$$
\begin{aligned}
j: X & \rightarrow X^{\odot *} \\
x \mapsto j x: X^{\odot} & \rightarrow \mathbb{C} \\
x^{\odot} & \mapsto\left\langle j x, x^{\odot}\right\rangle=\left\langle x^{\odot}, x\right\rangle
\end{aligned}
$$

Além disso, temos

$$
T^{\odot *}(t) j x=j(T(t) x)
$$

e portanto $j(X) \subset X^{\odot \odot}$. De fato, dado $x \in X$, como $T(t)$ é um $\mathcal{C}_{0}$-semigrupo então dado $\epsilon>0$, existe $\delta>0$ tal que para $|t|<\delta$, temos $\|T(t) x-x\|<\epsilon$. Portanto, usando (2.4) junto com a continuidade da $j$ obtemos

$$
\left\|T^{\odot *}(t) j(x)-j(x)\right\|=\|j(T(t) x)-j(x)\|<\epsilon .
$$

Consequentemente, $j(x) \in X^{\odot \odot}$.

Assim, todo $x \in X$ pode ser "tomado" como um elemento de $X^{\odot *}$, pelo mergulho $j$. Faremos isto no resto do texto, sem mencionar mais $j$. Assim $X \subset X^{\odot *}$. 
Definição 2.1. X é chamado $\odot$-reflexivo em relação a $A$ se $j(X)=X^{\odot \odot}$. E se omitirmos $j, X=X^{\odot \odot}$.

Algumas vezes é conveniente representar a inter-relação dos espaços esquematicamente no seguinte diagrama de "dualidade"

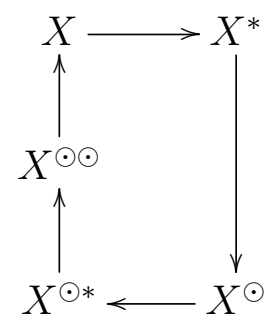

onde as setas horizontais indicam tomar adjunto e as setas verticais indicam tomar restrições ao espaço maximal onde acontece a continuidade forte. No caso $\odot$-reflexivo temos o seguinte diagrama simplificado

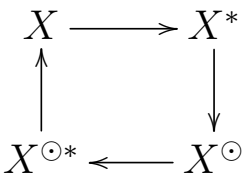

\subsection{O problema protótipo}

Nesta seção, estudaremos uma EDFR trivial, como sendo $\dot{x}=0$. Exibiremos com detalhe os semigrupos fortemente contínuos e seus adjuntos para este problema concreto. Para maiores informações, o leitor poderá consultar o livro do Diekmann et al [24, Capitulo II].

Consideremos o espaço de Banach $X=C([-h, 0], \mathbb{C})$ com a norma do supremo

$$
\|f\|_{X}=\sup _{x \in[-h, 0]}|f(x)| .
$$

Estudaremos o problema de valor inicial

$$
\begin{aligned}
\dot{x}(t) & =0, \text { para } t \geqslant 0, \\
x_{0} & =\varphi, \varphi \in X .
\end{aligned}
$$

A solução desta EDF é trivial e seu semigrupo solução é dado por

$$
\left(T_{0}(t) \varphi\right)(\theta)=\left\{\begin{array}{l}
\varphi(t+\theta), \quad \text { if }-h \leqslant t+\theta \leqslant 0 \\
\varphi(0), \quad \text { if } t+\theta \geqslant 0
\end{array}\right.
$$




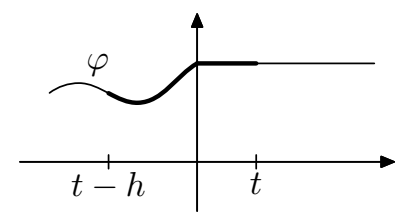

Figura 2.1: A solução de (2.6)

Podemos estender $\varphi$ para $[-h, \infty)$ pondo $\varphi(\theta)=\varphi(0), \theta \geqslant 0$ Em outras palavras $T_{0}(t)$ é um semigrupo translação. Além disso, a família $\left\{T_{0}(t)\right\}_{t \geqslant 0}$ é um $\mathcal{C}_{0}$-semigrupo. Com efeito,

- $\left(T_{0}(0) \varphi\right)(\theta)=\varphi(\theta)$, para $\theta \in[-h, 0]$, donde $T_{0}(0)=I$

- Para $t, s \geqslant 0$,

$$
\begin{aligned}
T_{0}(t)\left(T_{0}(s) \varphi\right)(\theta) & = \begin{cases}\varphi(t+s+\theta), & -h \leqslant t+s+\theta \leqslant 0 \\
\varphi(0), & t+s+\theta \geqslant 0\end{cases} \\
& =\left(T_{0}(t+s) \varphi\right)(\theta) .
\end{aligned}
$$

- Como $\left(T_{0}(t) \varphi-\varphi\right)(\theta)=\varphi(t+\theta)-\varphi(\theta)$, se $t+\theta \leqslant 0$, e como $\varphi$ é uniformemente contínua, temos

$$
\lim _{t \rightarrow 0}\left\|T_{0}(t) \varphi-\varphi\right\|=0
$$

No seguinte lema, explicitaremos o gerador infinitesimal $A_{0}$ do semigrupo translação $T_{0}(t)$.

Lema 2.2 (Vide [24, Lema II.2.1]). O gerador infinitesimal de $T_{0}$ é dado por

$$
\mathcal{D}\left(A_{0}\right)=\{\varphi \mid \dot{\varphi} \in C([-h, 0], \mathbb{C}), \dot{\varphi}(0)=0\}, \quad A_{0} \varphi=\dot{\varphi} .
$$

Na sequência, exibiremos o adjunto do semigrupo $T_{0}(t)$. Para isso é necessário observar que, quando $X=C([-h, 0], \mathbb{C})$, podemos identificar $X^{*}$ com o espaço das funções de variação limitada normalizadas, $\operatorname{NBV}([0, h], \mathbb{C})$. Consideraremos como domínio essencial das funções de variação limitada o intervalo $[0, h]$ no lugar de $[-h, 0]$. De fato, devemos estender o domínio da definição da função $f \in \operatorname{NBV}([0, h], \mathbb{C})$ a todo $\mathbb{R}$, considerando $f(\theta)=0$ para $\theta \leqslant 0$ e $f(\theta)=f(h)$ para $\theta \geqslant h$. 
Além disso, escreveremos a dualidade, para $f \in X^{*}$, e $\varphi \in X$, mediante uma integral de Riemann-Stieltjes

$$
\langle f, \varphi\rangle=\int_{0}^{h} d f(\theta) \varphi(-\theta)
$$

e algumas vezes, apesar de que $\varphi$ pode não estar definida para $\theta<-h$

$$
\langle f, \varphi\rangle=\int_{0}^{\infty} d f(\theta) \varphi(-\theta) .
$$

Observação 2.3. Note que os valores de $\varphi$ para $\theta<-h$ não importam, desde que $f$ é constante para $\theta \geqslant h$.

O próximo lema mostrará que o semigrupo adjunto de $T_{0}(t), T_{0}^{*}(t)$, é também um semigrupo translação.

Lema 2.4. Seja $T_{0}(t)$ o semigrupo definido em (2.8), o seu semigrupo adjunto é definido por $\left(T_{0}^{*}(t) f\right)(\sigma)=f(t+\sigma)$, para $\sigma>0$ e $f \in X^{*}=N B V$.

Demonstração. Sejam $f \in \mathrm{NBV}$ e $\varphi \in X$,

$$
\begin{aligned}
\left\langle f, T_{0}(t) \varphi\right\rangle & =\int_{0}^{\infty} d f(\theta)\left(T_{0}(t) \varphi\right)(-\theta) \\
& =\int_{0}^{t} d f(\theta) \varphi(0)+\int_{t}^{\infty} d f(\theta) \varphi(t-\theta) \\
& =f(t) \varphi(0)+\int_{0}^{\infty} d_{\sigma} f(t+\sigma) \varphi(-\sigma) .
\end{aligned}
$$

Portanto,

$$
\left\langle T_{0}^{*}(t) f, \varphi\right\rangle=\int_{0}^{\infty} d\left(T_{0}^{*}(t) f(\sigma)\right) \varphi(-\sigma)=f(t) \varphi(0)+\int_{0}^{\infty} d_{\sigma} f(t+\sigma) \varphi(-\sigma) .
$$

Devido as nossas convenções, os elementos de $X^{*}$ são zero para $\sigma \leqslant 0$ e contínuos a direita em $(0, h)$, então temos que $T_{0}^{*}(t) f$ tem um salto de magnitude $f(t)$ em $\sigma=0$ e este salto contribui o termo $f(t) \varphi(0)$, consequentemente

$$
\left(T_{0}^{*}(t) f\right)(\sigma)=f(t+\sigma), \quad \sigma>0 .
$$

Isto é, $T_{0}^{*}(t)$ é um semigrupo translação. 
Assim, observamos a vantagem de considerar $[0, h]$ no lugar de $[-h, 0]$ como o domínio de definição das funções NBV.

Observação 2.5. Da teoria geral temos que o semigrupo $T_{0}^{*}(t)$ é fraco-* contínuo (vide [7, Proposição 1.4.3]). Contudo se munirmos $X^{*}$ com a norma da topologia usual, observemos que $T_{0}^{*}(t)$ não é um $\mathcal{C}_{0}$-semigrupo. Com efeito, se escolhermos $f(\sigma)=0$ para $\sigma<h$ e $f(h) \neq 0$ então

$$
\left\|T_{0}^{*}(t) f-f\right\|_{B V}=2|f(h)| \neq 0 .
$$

A seguir, explicitaremos o domínio do gerador infinitesimal $A_{0}^{*}$ do semigrupo translação $T_{0}^{*}(t)$, e apresentaremos o subespaço maximal invariante onde tal semigrupo é fortemente contínuo.

Teorema 2.6 (Vide [24, Teorema II.5.1]). $f \in \mathcal{D}\left(A_{0}^{*}\right)$ se, e somente se, para $\theta>0$

$$
f(\theta)=f(0+)+\int_{0}^{\theta} g(\sigma) d \sigma,
$$

onde $g \in N B V \operatorname{com} g(h)=0$; para tal $f$ temos $A_{0}^{*} f=g$.

Agora, lembremos que o subespaço $X^{\odot}=\overline{\mathcal{D}\left(A_{0}^{*}\right)}$ é o subespaço maximal invariante onde $T_{0}^{*}(t)$ é um $\mathcal{C}_{0}$-semigrupo. Portanto, precisamos caracterizar o fecho de $\mathcal{D}\left(A_{0}^{*}\right)$. Neste sentido, observemos que as funções de variação limitada são densas em $L^{1}$ e a norma da variação total de $f(t)=f(0+)+$ $\int_{0}^{t} g(\theta) d \theta$ é igual a

$$
|f(0+)|+\|g\|_{L^{1}}
$$

Consequentemente, quando tomamos o fecho, a forma em (2.10) é conservada, porém $g$ é permitido estar em $L^{1}$. Em outras palavras, o fecho de $\mathcal{D}\left(A_{0}^{*}\right)$ consiste das funções que são absolutamente contínuas em $(0, h]$, não obstante podem ter salto em zero. Isto é o que nos diz o teorema a seguir.

Teorema 2.7 (Vide [24, Teorema II.5.2]). Para o semigrupo translação $T_{0}^{*}(t)$, temos

$$
\begin{aligned}
X^{\odot}=\overline{\mathcal{D}\left(A_{0}^{*}\right)}=\{f \in N B V \mid f(t) & =c+\int_{0}^{t} g(\theta) d \theta, t>0, c \in \mathbb{C}, \\
& \left.g \in L^{1}, g(\theta)=0 \text { para quase todo } \theta \geqslant h\right\}
\end{aligned}
$$

Observemos que os elementos de $X^{\odot}$ estão completamente especificados por $c \in \mathbb{C}$ e $g \in L^{1}$ e notemos que $f(0+)=c$. Mais precisamente, o espaço $X^{\odot}$ é isomorfo a $\mathbb{C} \times L^{1}([0, h], \mathbb{C})$ munido com a norma

$$
\|(c, g)\|=|c|+\|g\|_{L^{1}}
$$


No que segue, trabalharemos com esta representação para $X^{\odot}$, entretanto adotamos por convenção que a função em $L^{1}$ é estendida a $(h, \infty)$ por zero. Assim, definimos o semigrupo $T_{0}^{\odot}(t)=\left.T_{0}^{*}\right|_{X \odot}$ cuja representação é dada por

$$
T_{0}^{\odot}(t)(c, g)=\left(c+\int_{0}^{t} g(\sigma) d \sigma, g(t+\cdot)\right)
$$

Usando o Teorema 2.6, da Equação (2.12), e dos resultados conhecidos sobre geradores de translações em $L^{1}$ (vide [24, Apêndice II]), enunciamos o seguinte teorema que exibe o domínio do gerador infinitesimal $A_{0}^{\odot}$ do $\mathcal{C}_{0^{-}}$ semigrupo $T_{0}^{\odot}(t)$.

Teorema 2.8 (Vide [24, Teorema II.5.3]). Para o semigrupo translação $T_{0}^{\odot}(t)$,

$$
\begin{aligned}
\mathcal{D}\left(A_{0}^{\odot}\right)=\left\{f \mid f(t)=c+\int_{0}^{t} g(\theta) d \theta \text { para } t>0, \text { onde } c\right. & \in \mathbb{C} \\
& \text { e } g \in A C(0, h) \text { com } g(\theta)=0 \text { para } \theta \geqslant h\}
\end{aligned}
$$

e $A_{0}^{\odot} f=g$. Alternativamente, escrevemos

$$
\begin{gathered}
\mathcal{D}\left(A_{0}^{\odot}\right)=\{(c, g) \mid c \in \mathbb{C} \text { eg } \in A C(0, h) \operatorname{com} g(\theta)=0, \operatorname{com} \theta \geqslant h\}, \\
A_{0}^{\odot}(c, g)=(g(0+), \dot{g}) .
\end{gathered}
$$

Portanto, representaremos $X^{\odot *}$ por $\mathbb{C} \times L^{\infty}([-h, 0], \mathbb{C})$.

Observação 2.9. Vale a pena ressaltar que

1. A norma sobre $X^{\odot}$ dada por (2.11) permite mostrar que

$$
\|(\alpha, \varphi)\|=\sup \left\{|\alpha|,\|\varphi\|_{\infty}\right\}
$$

é uma norma em $X^{\odot *}=\mathbb{C} \times L^{\infty}([-h, 0], \mathbb{C})$ e a dualidade entre $X^{\odot *}$ e $X^{\odot}$ é dado por

$$
\langle(\alpha, \varphi),(c, g)\rangle=\alpha c+\int_{0}^{h} \varphi(-\theta) g(\theta) d \theta .
$$

2. Da fórmula explícita $(2.12)$ para $T_{0}^{\odot}$ deduzimos que

$$
T_{0}^{\odot *}(t)(\alpha, \varphi)=\left(\alpha, \varphi_{t}^{\alpha}\right)
$$


onde por definição

$$
\varphi_{t}^{\alpha}(\theta)= \begin{cases}\varphi(t+\theta), & t+\theta \leqslant 0 \\ \alpha, & t+\theta>0\end{cases}
$$

Com efeito,

$$
\begin{aligned}
\left\langle(\alpha, \varphi), T_{0}^{\odot}(t)(c, g)\right\rangle & =\left\langle(\alpha, \varphi),\left(c+\int_{0}^{t} g(\sigma) d \sigma, g(t+\cdot)\right)\right\rangle \\
& =\alpha c+\alpha \int_{0}^{t} g(\sigma) d \sigma+\int_{0}^{h} \varphi(-\theta) g(t+\theta) d \theta \\
& =\alpha c+\alpha \int_{0}^{t} g(\sigma) d \sigma+\int_{t}^{t+h} \varphi(t-\gamma) g(\gamma) d \gamma \\
& =\langle(\alpha, \psi),(c, g)\rangle
\end{aligned}
$$

onde

$$
\begin{aligned}
& \psi(-\theta)=\alpha, \quad \theta<t, \\
& \psi(-\theta)=\varphi(t-\theta), \quad \theta \geqslant t .
\end{aligned}
$$

Portanto, $\psi=\varphi_{t}^{\alpha}$, e observemos que $\varphi_{t}^{\alpha}$ é obtido estendendo $\varphi \in L^{\infty}$ com o valor de $\alpha$ para $\theta>0$ e transladando em $t$.

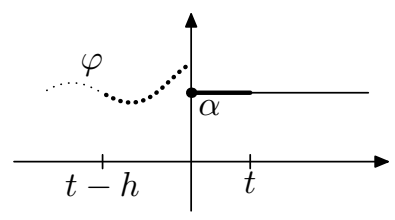

Figura 2.2: Interpretação gráfica de $\varphi_{t}^{\alpha}$, em negrito.

Finalmente, apresentaremos o domínio de definição do gerador infinitesimal $A_{0}^{\odot *}$ do semigrupo adjunto $T_{0}^{\odot *}(t)$. No próximo teorema, observaremos como o domínio fica independente da EDFR, a qual é transferida na ação do gerador.

Teorema 2.10 (Vide [24, Teorema II.5.5]). Para o semigrupo translação temos

$$
\mathcal{D}\left(A_{0}^{\odot *}\right)=\{(\alpha, \varphi) \mid \varphi \in \operatorname{Lip}(\alpha)\}, \quad A_{0}^{\odot *}(\alpha, \varphi)=(0, \dot{\varphi}),
$$

onde $\operatorname{Lip}(\alpha)$ denota o subespaço de $L^{\infty}([-h, 0], \mathbb{C})$ cujos elementos contem as funções Lipschitz contínuas que assumem o valor $\alpha$ em $\theta=0$. 
Observação 2.11. Quando tomamos o fecho do domínio de $A_{0}^{\odot *}$ perdemos a condição de Lipschitz das funções $\varphi$, porém permanece a continuidade. Portanto,

$$
X^{\odot \odot}=\overline{\mathcal{D}\left(A_{0}^{\odot *}\right)}=\{(\alpha, \varphi) \mid \varphi \in C(\alpha)\}
$$

onde $C(\alpha)$ denota o subespaço fechado de $L^{\infty}([-h, 0], \mathbb{C})$ cujos elementos são funções contínuas tais que em zero tomam o valor de $\alpha$.

Observemos que o mergulho $j$ de $X$ em $X^{\odot *}$ é tal que $j(\varphi)=(\varphi(0), \varphi)$. Portanto, $j(X)=X^{\odot \odot}$, ou seja, $X$ é $\odot$-reflexivo com respeito ao semigrupo translação $T_{0}$. Assim, podemos identificar elementos $\varphi \in X=C([-h, 0], \mathbb{C})$ com os elementos

$$
(\varphi(0), \varphi) \in X^{\odot \odot}=\left\{(\alpha, \varphi) \in \mathbb{C} \times L^{\infty}([-h, 0], \mathbb{C}) \mid \varphi \in C(\alpha)\right\}
$$

Falando a grosso modo, isto significa que os elementos de $X$ e $X^{\odot \odot}$ são os mesmos objetos.

Além disso, ressaltamos que, no espaço $X^{\odot *}$, a componente em $\mathbb{C}$ destaca a informação na qual a extensão é baseada. A equação diferencial

$$
\frac{d}{d t} x_{t}=A_{0}^{\odot *} x_{t}
$$

tem zero na componente $\mathbb{C}$, pois a extensão é constante: $\varphi(0)$.

Portanto, se mudarmos a regra da extensão, devemos mudar a equação diferencial somente na componente $\mathbb{C}$, no espaço $X^{\odot *}$. Na próxima seção, aplicaremos este estudo, para escrever uma EDFR qualquer como perturbação da EDFR trivial (problema protótipo).

Observação 2.12 (O sistema protótipo). Se considerarmos o sistema de equações

$$
\dot{x}(t)=0, \quad \text { para } x(t) \in \mathbb{C}^{n}
$$

como um EDFR, com espaço de fase $X=C\left([-h, 0], \mathbb{C}^{n}\right)$, como não há acoplamento, temos $n$ copias idênticas do caso escalar. Portanto, todos os resultados anteriores são válidos imediatamente para o sistema, simplesmente substituindo $\mathbb{C}$ por $\mathbb{C}^{n}$. É claro que acoplamentos podem surgir assim que mudarmos a regra para a extensão e então $\mathbb{C}^{n}$ torna-se importante. Portanto, nas seções posteriores começaremos trabalhando com sistemas desde o começo. 


\subsection{EDFRs lineares como perturbações limitadas}

Nesta seção, consideraremos a EDFR linear autônoma

$$
\dot{x}(t)=L x_{t} \quad t \geqslant 0, x(t) \in \mathbb{C}^{n}
$$

com condição inicial

$$
x(\theta)=\varphi(\theta), \quad \theta \in[-h, 0],
$$

onde $\varphi \in X=C\left([-h, 0], \mathbb{C}^{n}\right), L: X \rightarrow \mathbb{C}^{n}$ é uma aplicação linear contínua dada, como no Teorema da Representação de Riesz,

$$
L \varphi=\int_{0}^{h} d \zeta(\theta) \varphi(-\theta) .
$$

A fim de representar a Equação (2.14) como uma equação diferencial abstrata, usaremos as duas componentes: translação e extensão. Para isso, consideraremos o sistema protótipo, estudado com detalhe na Seção 2.2. Gostaríamos de escrever (2.14) na forma

$$
\frac{d}{d t} x_{t}=\left(A_{0}^{\odot *}+B\right) x_{t}
$$

onde, segundo o Teorema $2.10, A_{0}^{\odot *}(\alpha, \varphi)=(0, \dot{\varphi})$ e $B: X \rightarrow X^{\odot *}$ é definido por

$$
B \varphi=(L \varphi, 0) .
$$

Agora, observemos que $\operatorname{Im}\left(A_{0}^{\odot *}\right) \cap \operatorname{Im}(B)=\{(0,0)\}$. Portanto as componentes translação e extensão são independentes. Além disso, notemos que o operador $B$, que descreve a regra de extensão, além de ser linear, é limitado. Com efeito, seja $\varphi \in X$, como $L$ uma aplicação linear contínua existe $K>0$ tal que $|L \varphi| \leqslant K\|\varphi\|$. Portanto,

$$
\|B \varphi\|=\|(L \varphi, 0)\|=\sup \{|L \varphi|,\|0\|\} \leqslant K\|\varphi\| .
$$

Esta propriedade se deve ao fato que o espaço imagem, $X^{\odot *}$, é grande suficiente. Observemos também que, a pesar de conceber $x_{t}$ como elemento de $X$, a equação diferencial abstrata (2.17) é uma identidade para elementos de $X^{\odot *}$. 
O objetivo desta seção é mostrar que o semigrupo gerado por $A_{0}^{\odot *}+B$ é de fato o operador solução da EDFR autônoma (2.14), $T(t)$, definido pela fórmula da variação das constantes

$$
T(t) \varphi=T_{0}(t) \varphi+\int_{0}^{t} T_{0}^{\odot *}(t-\tau) B T(\tau) \varphi d \tau
$$

onde $T_{0}$ é o semigrupo translação dado na seção anterior e $B$ é um operador linear limitado. A ideia é perturbar o gerador infinitesimal $A_{0}$, do semigrupo $T_{0}$, pelo operado linear limitado $B$. Em seguida, mostraremos que os espaços $X^{\odot}$ e $X^{\odot \odot}$ são os mesmos para $T(t)$ e $T_{0}(t)$, como consequência da limitação de $B$. Em outras palavras, a estrutura de dualidade é preservada por perturbações limitadas. Dedicaremos duas subseções para o estudo das perturbações. Na primeira nos restringiremos ao caso $\odot$-reflexivo e na segunda descreveremos as perturbações limitadas com imagem de dimensão finita.

\subsubsection{Perturbações limitadas no caso $\odot$-reflexivo}

Ao longo desta subseção, $T_{0}$ continuará denotando o $\mathcal{C}_{0}$-semigrupo sobre o espaço de Banach $X$, considerado $\odot$-reflexivo com respeito a $T_{0}$. O gerador infinitesimal de $T_{0}$ é denotado por $A_{0}$. De fato o semigrupo translação é o exemplo motivante, não obstante os resultados terão maior aplicabilidade. Começaremos enunciando um resultado técnico, que involucra a definição de integral fraca-* tratada no Apêndice A.3.

Lema 2.13 (Vide [24, Lema III.2.3]). Seja $f: \mathbb{R}_{+} \rightarrow X^{\odot *}$ contínua na norma. Definamos $v: \mathbb{R}_{+} \rightarrow X^{\odot *}$ como a integral fraca-*

$$
v(t)=\int_{0}^{t} T_{0}^{\odot *}(t-\tau) f(\tau) d \tau .
$$

Então, $v$ é contínua na norma, toma valores em $X$ e

$$
\|v(t)\| \leqslant M \frac{e^{\omega t}-1}{\omega} \sup _{0 \leqslant \tau \leqslant t}\|f(\tau)\|
$$

onde $M$ e $\omega$ são tal que $\left\|T_{0}(t)\right\| \leqslant M e^{\omega t}$. Além disso,

$$
\frac{1}{t} v(t) \stackrel{*}{\rightarrow} f(0), \quad \text { quando } t \downarrow 0,
$$

onde $\stackrel{*}{\rightarrow}$ representa a convergência fraca-*. 
Teorema 2.14 (Vide [24, Teorema III.2.4]). Seja $B: X \rightarrow X^{\odot *}$ um operador linear e limitado. Existe um único $\mathcal{C}_{0}$-semigrupo $T(t)$ que satisfaz (2.19).

O próximo passo é determinar o gerador infinitesimal de $T(t)$, que será denotado por $A$. Para isso, uma abordagem mais simples segue de forma indireta: determinar $A^{*}, A^{\odot}, A^{\odot *}$. Para começar, devemos verificar também que $X^{\odot}$ é o espaço onde $T^{*}$ é um $\mathcal{C}_{0}$-semigrupo.

Defina $U(t)$ como a diferença do semigrupo perturbado $T(t)$, do semigrupo não perturbado $T_{0}(t)$, isto é

$$
U(t) \varphi:=T(t) \varphi-T_{0}(t) \varphi=\int_{0}^{t} T_{0}^{\odot *}(t-\tau) B T(\tau) d \tau
$$

e de acordo com o Lema 2.13, $\|U(t)\| \rightarrow 0$ quando $t \downarrow 0$. Segue também que $\left\|U^{*}(t)\right\| \rightarrow 0$. Consequentemente temos o seguinte resultado.

Lema 2.15 (Vide [24, Lema III.2.6]). O subespaço $X^{\odot}$, primeiro definido em termos do semigrupo não perturbado $T_{0}^{*}$, também é o subespaço onde $T^{*} e ́$ um $\mathcal{C}_{0}$-semigrupo. Em particular $X^{\odot}$ é invariante por $T^{*}$.

O adjunto de $B, B^{*}$, aplica o espaço $X^{\odot * *}$ em $X^{*}$. O espaço de Banach $X^{\odot}$ pode ser considerado como um subespaço do seu segundo espaço dual, $X^{\odot * *}$ e consequentemente podemos restringir $B$ a $X^{\odot}$. Deveríamos introduzir um símbolo novo para esta restrição, porém simplesmente escreveremos

$$
B^{*}: X^{\odot} \rightarrow X^{*}
$$

Lema 2.16 (Vide [24, Lema III.2.7]). Para $x^{\odot} \in X^{\odot}$ e $x \in X$, com $U$ dado em (2.21),

$$
\frac{1}{t}\left\langle U^{*} x^{\odot}, x\right\rangle \rightarrow\left\langle B^{*} x^{\odot}, x\right\rangle, \quad \text { quando } \quad t \downarrow 0 .
$$

Agora, lembremos que de acordo com a teoria geral

$$
\mathcal{D}\left(A^{*}\right)=\left\{x^{*} \mid \frac{1}{t}\left(T^{*}(t) x^{*}-x^{*}\right) \text { converge fraco-* quando } t \downarrow 0\right\}
$$

e para cada $x \in \mathcal{D}\left(A^{*}\right)$

$$
\frac{1}{t}\left(T^{*}(t) x^{*}-x^{*}\right) \stackrel{*}{\rightarrow} A^{*} x^{*}, \quad \text { quando } \quad t \downarrow 0 .
$$


Corolário 2.17 (Vide [24, Corolário III.2.8]). $\mathcal{D}\left(A^{*}\right)=\mathcal{D}\left(A_{0}^{*}\right)$ e $A^{*}=A_{0}^{*}+B^{*}$.

Agora, denotemos $T^{\odot}(t)=\left.T^{*}(t)\right|_{X \odot}$, e o gerador infinitesimal de $T^{\odot}$ é a parte de $A_{0}^{*}+B^{*}$ em $X^{\odot}$.

Corolário 2.18 (Vide [24, Corolário III.2.9]). O $\mathcal{C}_{0}$-semigrupo $T^{\odot}(t)$ é gerado pelo operador $A^{\odot}$ definido por

$$
\mathcal{D}\left(A^{\odot}\right)=\left\{x^{\odot} \in \mathcal{D}\left(A_{0}^{*}\right) \mid\left(A_{0}^{*}+B^{*}\right) x^{\odot} \in X^{\odot}\right\}, \quad A^{\odot}=A_{0}^{*}+B^{*} .
$$

Sabemos que ambos $T_{0}^{*}$ e $T^{*}$ deixam invariante $X^{\odot}$. Considerando $x^{\odot} \in X^{\odot}$,

$$
U^{*}(t) x^{*}=T^{*} x^{\odot}-T_{0}^{*} x^{\odot} \in X^{\odot} .
$$

Portanto, $U^{*}$ também deixa invariante $X^{\odot}$. Denotemos $U^{\odot}$ a restrição de $U^{*}$ a $X^{\odot}$, então $\left\|U^{\odot}(t)\right\| \rightarrow 0$ quando $t \downarrow 0$, assim como $\left\|U^{\odot *}(t)\right\| \rightarrow 0$. Consequentemente, $t \mapsto T^{\odot *}(t) x^{*}$ é contínua na norma em $t=0$ se e somente se, $t \mapsto T_{0}^{\odot *}(t) x^{*}$ tem a mesma propriedade. Por conseguinte, podemos concluir, como foi feito anteriormente, que o espaço $X^{\odot \odot}$ é o mesmo para $T_{0}^{\odot *}$ e $T^{\odot *}$ e em particular temos o seguinte resultado.

Teorema 2.19 (Vide [24, Teorema III.2.10]). O espaço X é $\odot$-reflexivo com respeito ao semigrupo perturbado $T(t)$.

Como uma reformulação do Lema 2.16 temos

Lema 2.20 (Vide [24, Lema III.2.11]). Para $x \in X$ arbitrário e $x^{\odot} \in X^{\odot}$,

$$
\frac{1}{t}\left\langle x^{\odot}, U(t) x\right\rangle \rightarrow\left\langle B x, x^{\odot}\right\rangle
$$

Raciocinando como acima, deduzimos

Corolário 2.21 (Vide [24, Corolário III.2.12]). $\mathcal{D}\left(A^{\odot *}\right)=\mathcal{D}\left(A_{0}^{\odot *}\right)$ e $A^{\odot *}=$ $A_{0}^{\odot *}+B$

Portanto, cabe mencionar uma vez mais, que a teoria sol-estrela $(\odot *)$, permite mostrar que a EDFR, embutida em princípio no domínio do gerador infinitesimal do semigrupo dado pelo operador solução, é transferida para a ação do gerador $A^{\odot *}$ do semigrupo $T^{\odot *}$ 


\subsubsection{Perturbações limitadas com imagem de dimensão finita}

No caso das EDFRs, o operador perturbação $B$ tem imagem de dimensão finita. Nesta subseção, mostraremos as principais consequências dessa propriedade. Em particular, a fórmula da variação das constantes (2.19) será reduzida a uma equação de renovação e observaremos que a equação de renovação contém todas as informações relevantes, ainda quando a condição inicial é tomada num espaço "maior".

Suponhamos que a imagem do operador $B: X \rightarrow X^{\odot *}$ esteja contida num subespaço de dimensão $n<\infty$, gerado pelos elementos linearmente independentes $r_{1}^{\odot *}, \ldots, r_{n}^{\odot *} \in X^{\odot *}$. Então, existem $r_{1}^{*}, \ldots, r_{n}^{*} \in X^{*}$ tais que

$$
B \varphi=\sum_{i=1}^{n}\left\langle r_{i}^{*}, \varphi\right\rangle r_{i}^{\odot *}
$$

Consequentemente, na fórmula da variação das constantes (2.19), obtemos

$$
\begin{aligned}
T(t) \varphi & =T_{0}(t) \varphi+\int_{0}^{t} T_{0}^{\odot *}(t-\tau) \sum_{i=1}^{n}\left\langle r_{i}^{*}, T(\tau) \varphi\right\rangle r_{i}^{\odot *} d \tau \\
& =T_{0}(t) \varphi+\sum_{i=1}^{n} \int_{0}^{t} T_{0}^{\odot *}(t-\tau)\left\langle r_{i}^{*}, T(\tau) \varphi\right\rangle r_{i}^{\odot *} d \tau
\end{aligned}
$$

Considerando a função $y:[0, \infty) \rightarrow \mathbb{C}^{n}$, cujas componentes são definidas por

$$
y_{i}(t)=\left\langle r_{i}^{*}, T(t) \varphi\right\rangle
$$

podemos rescrever (2.22) como

$$
T(t) \varphi=T_{0}(t) \varphi+\sum_{i=1}^{n} \int_{0}^{t} T_{0}^{\odot *}(t-\tau) y_{i}(t) r_{i}^{\odot *} d \tau
$$

Assim, obteremos uma expressão explícita para $T(t) \varphi$, uma vez que y seja conhecida.

Na tentativa de obter uma equação para $y$, aplicaríamos $r_{i}^{*}$ a ambos os lados de (2.23). Porém, notemos que, não é possível inverter imediatamente a ordem de aplicar $r_{i}^{*}$ e conseguir a integração, uma vez que, em geral

$$
\left\langle r_{i}^{*}, T_{0}^{\odot *}(t) r_{j}^{\odot *}\right\rangle
$$


não esta definido. No entanto, a função

$$
t \mapsto\left\langle r_{i}^{*}, T_{0}^{\odot *}(t) r_{j}^{\odot *}\right\rangle
$$

existe no sentido $L_{\text {loc }}^{\infty}$, como será mostrado depois, e portanto podemos intercambiar a ordem e obteremos uma equação de renovação para $y$. Para tais fins, precisamos de algumas preparações técnicas.

Seja

$$
W(t) x^{\odot *}=\int_{0}^{t} T^{\odot *}(\tau) x^{\odot *} d \tau
$$

Vale a pena mencionar que $W_{0}$ é definido analogamente com $T_{0}^{\odot *}$.

Lema 2.22 (Vide [24, Lema III.3.1]). Para $x^{*} \in X^{*}$ e $x^{\odot *} \in X^{\odot *}$ a função

$$
t \mapsto\left\langle x^{*}, W(t) x^{\odot *}\right\rangle
$$

é localmente Lipschitz contínua e consequentemente tem derivada bem definida no sentido de $L_{l o c}^{\infty}$.

Corolário 2.23 (Vide [24, Corolario III.3.2]). Existe uma função matricial $R$ de ordem $n \times n$, cujas entradas satisfazem

$$
\int_{0}^{t} R_{i j}(\tau) d \tau=\left\langle r_{i}^{*}, W(t) r_{j}^{\odot *}\right\rangle, \quad t \geqslant 0 .
$$

Lembremos que para qualquer $\zeta \in L^{1}$, a integral fraca-* (definida no apêndice A.3)

$$
\int_{0}^{t} T_{0}^{\odot *}(t-\tau) \zeta(\tau) x^{\odot *} d \tau
$$

define uma função contínua em $t$, que toma valores em $X$. Além disso, $R_{0}$ é a equivalente a $R$ para $W_{0}$.

Lema 2.24 (Vide [24, Lema III.3.3]). Para qualquer $\zeta \in L^{1}$, a seguinte identidade é satisfeita

$$
\left\langle r_{i}^{*}, \int_{0}^{t} T_{0}^{\odot *}(t-\tau) \zeta(\tau) r_{j}^{\odot *} d \tau\right\rangle=\int_{0}^{t} R_{0 i j}(t-\tau) \zeta(\tau) d \tau
$$


Corolário 2.25 (Vide [24, Corolario III.3.4]). Para $x^{\odot *} \in X^{\odot *}$ defina a função $n$-vetorial $y$, com elementos em $L_{l o c}^{\infty}$, pela relação

$$
\int_{0}^{t} y_{i}(\tau) d \tau=\left\langle r_{i}^{*}, W(t) x^{\odot *}\right\rangle
$$

Então

$$
y=y_{0}+R_{0} * y
$$

onde $R_{0} * y$ denota o produto por convolução de $R_{0}$ com $y$,

$$
y_{0 i}(t)=\frac{d}{d t}\left\langle r_{i}^{*}, W_{0}(t) x^{\odot *}\right\rangle, \quad R_{0 i j}(t)=\frac{d}{d t}\left\langle r_{i}^{*}, W_{0}(t) r_{j}^{\odot *}\right\rangle .
$$

Corolário 2.26 (Vide [24, Corolario III.3.5]). O núcleo $R$ é o resolvente do núcleo $R_{0}$, isto é,

$$
R=R_{0}+R_{0} * R
$$

O próximo teorema mostra que dada qualquer solução $y$ de (2.28), podemos recuperar $T(t) x$, por

$$
T(t) x=T_{0}(t) x+\sum_{j=1}^{n} \int_{0}^{t} T_{0}^{\odot *}(t-\tau) r_{j}^{\odot *} y_{j}(\tau) d \tau .
$$

Em consequência notamos que resolver (2.19) se reduz a resolver a equação (2.28).

Teorema 2.27 (Vide [24, Teorema III.3.6]). O $\mathcal{C}_{0}$-semigrupo $T^{\odot *}$ tem a seguinte representação

$$
T^{\odot *}(t) x^{\odot *}=T_{0}^{\odot *} x^{\odot *}+\sum_{j=1}^{n} \int_{0}^{t} T_{0}^{\odot * *}(t-\tau) y_{j}(\tau) r_{j}^{\odot *} d \tau .
$$

Corolário 2.28 (Vide [24, Corolário III.3.7]). Para todo $x^{\odot *} \in X^{\odot *}$ e $t \geqslant 0$,

$$
T^{\odot *}(t) x^{\odot *}-T_{0}^{\odot *}(t) x^{\odot *} \in X .
$$

Seja $B^{*}: X^{\odot} \rightarrow X^{*}$ o adjunto de $B$. O semigrupo $\left\{T^{\odot}(t)\right\}$ satisfaz a fórmula da variação dos parâmetros adjunta (para mais detalhes sobre as variantes da fórmula da variação dos parâmetros consulte o Apêndice A.4)

$$
T^{\odot}(t) x^{\odot}=T_{0}^{\odot}(t) x^{\odot}+\int_{0}^{t} T_{0}^{*}(t-\tau) B^{*} T^{\odot}(\tau) x^{\odot} d \tau .
$$


Portanto, se

$$
B^{*} x^{\odot}=\sum_{i=1}^{n} r_{i}^{*}\left\langle x^{\odot}, r_{i}^{\odot *}\right\rangle
$$

então a função $n$-vetorial dada por

$$
z_{i}(t)=\left\langle T^{\odot}(t) x^{\odot}, r_{i}^{\odot *}\right\rangle
$$

satisfaz a equação de renovação adjunta

$$
z=z_{0}+R_{0}^{T} * z
$$

onde $R_{0}^{T}$ denota a transposta de $R_{0}$. Se as entradas são complexas então temos que tomar o conjugado complexo, e a função forçante é definida por

$$
z_{0 i}(t)=\left\langle T_{0}^{\odot}(t) x^{\odot}, r_{i}^{\odot *}\right\rangle
$$

Uma vez mais, podemos recuperar o semigrupo a partir de $z$ somente. Assim, finalizamos as subseções.

Voltando a nosso objetivo inicial, temos o seguinte teorema.

Teorema 2.29 (Vide [24, Teorema III.4.1]). Sejam $\left\{T_{0}(t)\right\}$ e $B$ definidos anteriormente, $\{T(t)\}$ um semigrupo definido pela integral abstrata,

$$
T(t) \varphi=T_{0}(t) \varphi+\int_{0}^{t} T_{0}^{\odot *}(t-\tau) B T(\tau) \varphi d \tau .
$$

Seja $x(t)$ a solução da EDFR

$$
\left\{\begin{array}{l}
\dot{x}(t)=L x_{t}, \quad t \geqslant 0 \\
x_{0}=\varphi
\end{array}\right.
$$

Então

$$
T(t) \varphi=x_{t}(\cdot ; \varphi)
$$

Vale a pena lembrar que estamos trabalhando com os espaços duais dados dados por $X^{*}=\operatorname{NBV}\left([0, h], \mathbb{C}^{n}\right), X^{\odot *}=\mathbb{C}^{n} \times L^{\infty}\left([-h, 0], \mathbb{C}^{n}\right)$. Além disso, lembremos também que o operador $B: X \rightarrow X^{\odot *}$ é definido por

$$
B \varphi=(L \varphi, 0), \quad L \varphi=\int_{0}^{h} d \zeta(\theta) \varphi(-\theta)
$$


e está exatamente na forma

$$
B \varphi=\sum_{i=1}^{n}\left\langle r_{i}^{*}, \varphi\right\rangle r_{i}^{\odot *}
$$

uma vez que seja considerado $r_{i}^{*} \in X^{*}$ como a $i$-ésima linha da função matricial NBV $\zeta$, e $r_{i}^{\odot *}=\left(e_{i}, 0\right) \in X^{\odot *}$, onde $e_{i}$ denota o $i$-ésimo vetor unitário em $\mathbb{C}^{n}$.

A prova do teorema está baseada na identificação da equação de renovação

$$
y=y_{0}+R_{0} * y
$$

derivada na Subseção 2.3.2, com uma equação de renovação correspondente à EDFR autônoma (2.33)

$$
\dot{x}=\zeta * \dot{x}+g, \quad g(t)=\zeta(t) \varphi(0)+\int_{t}^{h} d \zeta(\theta) \varphi(t-\theta)
$$

dada no livro de Diekmann et al [24, Seção I.2]. Para isto, precisamos de alguns lemas para elaborar os componentes da equação de renovação dada na subseção anterior. O primeiro passo é determinar o núcleo $R_{0}$.

\section{Lema 2.30.}

i) (Vide [24, Lema III.4.2]) Para $f \in X^{\odot},\left\langle r_{i}^{\odot *}, f\right\rangle=f_{i}(0+)$.

ii) (Vide [24, Lema III.4.3]) Para $\zeta \in L_{\text {loc }}^{1}$

$$
X^{\odot *} \ni \int_{0}^{t} T_{0}^{\odot *}(t-\tau) \zeta(\tau) r_{i}^{\odot *} d \tau=e_{i} \int_{0}^{\max \{0, t+\cdot\}} \zeta(\sigma) d \sigma .
$$

iii) (Vide [24, Corolário III.4.4]) $W_{0}(t) r_{i}^{\odot *}=e_{i} \max \{0, t+\cdot\}$.

iv) (Vide [24, Corolário III.4.5]) $R_{0}=\zeta$.

Agora, apresentaremos uma expressão para a integral da função forçante $y_{0}$ na equação de renovação (2.34).

Lema 2.31 (Vide [24, Lema III.4.6]). Para $x^{\odot *}=(\alpha, \varphi) \in \mathbb{C} \times L^{\infty}$

$$
\int_{0}^{t} y_{0}(\tau) d \tau=\int_{0}^{h} d \zeta(\theta) \int_{-\theta}^{t-\theta} \varphi^{\alpha}(\sigma) d \sigma
$$


onde por definição

$$
\varphi^{\alpha}(\theta)=\left\{\begin{array}{l}
\varphi(\theta), \quad-h \leqslant \theta \leqslant 0 \\
\alpha, \quad \theta>0
\end{array}\right.
$$

Quando $x^{\odot *} \in X$, isto é, $\varphi$ é contínua e $\alpha=\varphi(0)$, isto implica que

$$
y_{0}(t)=\zeta(t) \varphi(0)+\int_{t}^{h} d \zeta(\varphi) \varphi(t-\theta)
$$

Consequentemente, podemos identificar neste caso especial a equação de renovação

$$
y=y_{0}+R_{0} * y
$$

com a equação de renovação

$$
\dot{x}(t)=\int_{0}^{t} \zeta(\theta) \dot{x}(t-\theta) d \theta+g(t) .
$$

Combinando a representação geral

$$
T^{\odot *}(t) x^{\odot *}=T_{0}^{\odot *}(t) x^{\odot *}+\sum_{j=1}^{n} \int_{0}^{t} T_{0}^{\odot *}(t-\tau) y_{j}(\tau) r_{j}^{\odot *} d \tau
$$

com o Lema 2.30(ii), obtemos

$$
T^{\odot *}(t) x^{\odot *}-T_{0}^{\odot *}(t) x^{\odot *}=\int_{0}^{\max \{0, t+\cdot\}} y(\tau) d \tau
$$

Portanto, se denotamos a componente em $\mathbb{C}$ de $T^{\odot *}(t) x^{\odot *}$ por $x(t)$, então

$$
x(t)=\alpha+\int_{0}^{t} y(\tau) d \tau, \quad t \geqslant 0,
$$

e a componente em $L^{\infty}$,

$$
T^{\odot *}(t) x^{\odot *}=\left\{\begin{array}{l}
\varphi(t+\theta), \quad t+\theta \leqslant 0 \\
\alpha+\int_{0}^{t+\theta} y(\tau) d \tau=x(t+\theta)
\end{array}\right.
$$

o que mostra que $y=\dot{x}$ e que a ação do semigrupo $T^{\odot *}(t)$ corresponde na verdade à translação ao longo da solução da EDFR. Em particular, restringindo a condição inicial a elementos de $X$, obtemos $T(t) \varphi=x_{t}(\cdot ; \varphi)$, assim fica demonstrado o Teorema 2.29. 


\section{Capítulo 3}

\section{Equações Diferenciais Ordinárias Generalizadas}

Neste capítulo, apresentaremos algumas definições básicas e alguns resultados importantes sobre equações diferenciais ordinárias generalizadas (EDOs generalizadas).

Este capítulo está dividido em quatro seções. Na primeira delas, abordaremos a integral de Kurzweil, apresentando a definição desta integral, suas propriedades fundamentais e alguns resultados importantes. Na segunda seção, abordaremos as equações diferenciais ordinárias generalizadas, que são definidas a partir da integral de Kurzweil. Na terceira seção, analisaremos a existência e a unicidade de soluções de EDOs generalizadas. Finalmente, na quarta seção, abordaremos a dependência contínua das soluções com respeito aos parâmetros iniciais para as EDOs generalizadas.

As principais referências para este capítulo são $[2,28,63,74]$.

\subsection{A Integral de Kurzweil}

Consideremos um intervalo fechado $[a, b] \subset \mathbb{R},-\infty<a<b<+\infty$.

Uma divisão ou partição marcada do intervalo $[a, b] \subset \mathbb{R}$ é uma coleção finita

$$
\left\{\left(\tau_{j},\left[\alpha_{j-1}, \alpha_{j}\right]\right) \mid j=1,2, \ldots, k\right\}
$$

onde $a=\alpha_{0}<\alpha_{1}<\cdots<\alpha_{k}=b$ é uma divisão de $[a, b]$ e $\tau_{j} \in\left[\alpha_{j-1}, \alpha_{j}\right]$, $j=1,2, \ldots, k$ é marca. 
Uma divisão ou partição parcial marcada do intervalo $[a, b] \subset \mathbb{R}$ é uma coleção finita

$$
\left\{\left(\xi_{j},\left[\beta_{j}, \gamma_{j}\right]\right) \mid j=1,2, \ldots, m\right\}
$$

onde $a \leqslant \beta_{j} \leqslant \xi_{j} \leqslant \gamma_{j} \leqslant \beta_{j+1} \leqslant \xi_{j+1} \leqslant \gamma_{j+1} \leqslant b$, para $j=1, \ldots, m$. Observemos que toda divisão marcada é uma divisão parcial marcada.

Chamaremos de calibre em $[a, b]$, qualquer função positiva $\delta:[a, b] \rightarrow$ $(0,+\infty)$. Dado um calibre $\delta$ em $[a, b]$, diremos que uma divisão parcial marcada $D=\left\{\left(\tau_{j},\left[\beta_{j}, \gamma_{j}\right]\right) \mid j=1, \ldots, m\right\}$ de $[a, b]$ é $\delta$-fina, se

$$
\left[\beta_{j}, \gamma_{j}\right] \subset\left(\tau_{j}-\delta\left(\tau_{j}\right), \tau_{j}+\delta\left(\tau_{j}\right)\right)
$$

Sejam $X$ um espaço de Banach e $U:[a, b] \times[a, b] \rightarrow X$ uma função de duas variáveis $\tau, t \in[a, b]$. Usaremos a notação

$$
S(U, D)=\sum_{j=1}^{k}\left[U\left(\tau_{j}, \alpha_{j}\right)-U\left(\tau_{j}, \alpha_{j-1}\right)\right]
$$

para denotar a soma de tipo Riemann correspondente à função $U$ e à divisão parcial marcada $D=\left\{\tau_{j},\left[\alpha_{j-1}, \alpha_{j}\right] \mid j=1, \ldots, k\right\}$.

O primeiro resultado que apresentamos diz que, para cada calibre $\delta$ de $[a, b]$, sempre é possível obter uma partição $\delta$-fina de $[a, b]$. Este resultado é conhecido como Lema de Cousin e é importante para a definição da integral que apresentaremos na sequência.

Lema 3.1 (Vide [74, Lema 1.4]). Dado um calibre $\delta$ em $[a, b]$, existe uma partição $\delta$-fina $D=\left\{\tau_{j},\left[\alpha_{j-1}, \alpha_{j}\right] \mid j=1, \ldots, k\right\}$ de $[a, b]$.

Agora, apresentaremos a definição da integral de Kurzweil, devida ao matemático tcheco Jaroslav Kurzweil que generalizou a integral de Perron em seu artigo [56].

Definição 3.2. Diremos que $U:[a, b] \times[a, b] \rightarrow X$ é uma função Kurzweil integrável sobre o intervalo $[a, b]$, se existir um único elemento $I \in X$ tal que para todo $\epsilon>0$, existe um calibre $\delta$ em $[a, b]$ tal que

$$
\|S(U, D)-I\|=\left\|\sum_{j=1}^{k}\left[U\left(\tau_{j}, \alpha_{j}\right)-U\left(\tau_{j}, \alpha_{j-1}\right)\right]-I\right\|<\epsilon
$$

para toda partição $\delta$-fina

$$
D=\left\{\left(\tau_{j},\left[\alpha_{j-1}, \alpha_{j}\right]\right), j=1, \ldots, k\right\}=\left\{\alpha_{0}, \tau_{1}, \alpha_{1}, \ldots, \tau_{k}, \alpha_{k}\right\}
$$

de $[a, b]$. 
Na definição acima, chamaremos $I$ de integral de Kurzweil de $U$ sobre o intervalo $[a, b]$ e denotaremos esta integral por $\int_{a}^{b} D U(\tau, t)$.

Se $\int_{a}^{b} D U(\tau, t)$ existir, então definiremos $\int_{a}^{b} D U(\tau, t)=-\int_{b}^{a} D U(\tau, t) \mathrm{e}$ quando $a=b$, definiremos $\int_{a}^{b} D U(\tau, t)=0$.

Observação 3.3. Notemos que o Lema de Cousin garante a existência de uma partição $\delta$-fina do intervalo $[a, b]$, para um calibre $\delta$ dado. Assim, a Definição 3.2 faz sentido. Notemos, também, que a integral de RiemannStieltjes é claramente um caso particular de integral de Kurzweil quando $U(\tau, t)=f(\tau) g(t)$. Em particular, funções Riemann integráveis são integráveis no sentido de Kurzweil com função calibre constante.

Denotaremos por $\mathcal{K}([a, b], X)$ o conjunto de todas as funções $U:[a, b] \times$ $[a, b] \mapsto X$ que são integráveis no intervalo $[a, b]$, no sentido de Kurzweil.

A seguir, enunciaremos alguns teoremas que tratam de algumas propriedades fundamentais desta integral cujas provas podem ser encontradas no livro de Schwabik [74] para o caso $X=\mathbb{R}^{n}$ e na dissertação de Mesquita [63] no caso em que $X$ é um espaço de Banach. O primeiro teorema que apresentamos nos diz que a integral de Kurzweil é linear.

Teorema 3.4 (Vide $\left[74\right.$, Teorema 1.9] ). Se $U, V \in \mathcal{K}([a, b], X)$ e $c_{1}, c_{2} \in \mathbb{R}$, então $c_{1} U+c_{2} V \in \mathcal{K}([a, b], X) e$

$$
\int_{a}^{b} D\left[c_{1} U(\tau, t)+c_{2} V(\tau, t)\right]=c_{1} \int_{a}^{b} D U(\tau, t)+c_{2} \int_{a}^{b} D V(\tau, t) .
$$

A seguir, apresentamos o Critério de Cauchy para a integral de Kurzweil.

Teorema 3.5 (Vide $[74$, Teorema 1.7]). A função $U:[a, b] \times[a, b] \rightarrow X$ será integrável sobre $[a, b]$ se, e somente se, para todo $\epsilon>0$, existir um calibre $\delta$ em $[a, b]$ tal que

$$
\left\|S\left(U, D_{1}\right)-S\left(U, D_{2}\right)\right\|<\epsilon
$$

para quaisquer partições $\delta$-finas $D_{1}, D_{2}$ de $[a, b]$.

O próximo resultado trata da integrabilidade em subintervalos de $[a, b]$, a qual também é uma propriedade da integral de Riemann.

Teorema 3.6 (Vide $[74$, Teorema 1.10$])$. Se $U \in \mathcal{K}([a, b], X)$, então para todo $[c, d] \subset[a, b]$ teremos $U \in \mathcal{K}([c, d], X)$. 
Outras propriedades da integral de Riemann também valem para a integral de Kurzweil, como a propriedade usual de aditividade em subintervalos adjacentes, que é relatado no próximo resultado.

Teorema 3.7 (Vide [74, Teorema 1.11]). Se c $\in(a, b)$ e $U:[a, b] \times[a, b] \rightarrow X$ forem tais que $U \in \mathcal{K}([a, c], X)$ e $U \in \mathcal{K}([c, b], X)$, então $U \in \mathcal{K}([a, b], X)$ e

$$
\int_{a}^{b} D U(\tau, t)=\int_{a}^{c} D U(\tau, t)+\int_{c}^{b} D U(\tau, t) .
$$

O resultado que enunciaremos abaixo é conhecido como Lema de SaksHenstock e é devido ao polonês Stanislaw Saks e ao inglês Ralph Henstock. O lema diz que a integral em "partes" de $[a, b]$ corresponde à soma de Riemann relativamente à estas "partes" de $[a, b]$ para os mesmos valores de $\epsilon$ e $\delta$ da definição de integral sobre todo intervalo $[a, b]$.

Lema 3.8 (Vide [74, Lema 1.13]). Seja $U:[a, b] \times[a, b] \rightarrow X$ integrável sobre $[a, b]$. Dado $\epsilon>0$, seja $\delta$ uma função calibre em $[a, b]$ tal que

$$
\left\|\sum_{j=1}^{k}\left[U\left(\tau_{j}, \alpha_{j}\right)-U\left(\tau_{j}, \alpha_{j-1}\right)\right]-\int_{a}^{b} D U(\tau, t)\right\|<\epsilon
$$

para toda partição $\delta$-fina $D=\left\{\left(\tau_{j},\left[\alpha_{j-1}, \alpha_{j}\right]\right), j=1,2, \ldots, k\right\}$ de $[a, b]$. Se

$$
a \leqslant \beta_{1} \leqslant \xi_{1} \leqslant \gamma_{1} \leqslant \beta_{2} \leqslant \xi_{2} \leqslant \gamma_{2} \leqslant \ldots \leqslant \beta_{m} \leqslant \xi_{m} \leqslant \gamma_{m} \leqslant b
$$

representar uma divisão $\delta$-fina $\left\{\left(\xi_{j},\left[\beta_{j}, \gamma_{j}\right]\right), j=1,2, \ldots, m\right\}$, isto é,

$$
\xi_{j} \in\left[\beta_{j}, \gamma_{j}\right] \subset\left(\xi_{j}-\delta\left(\xi_{j}\right), \xi_{j}+\delta\left(\xi_{j}\right)\right), \quad j=1,2, \ldots, m,
$$

então teremos

$$
\left\|\sum_{j=1}^{m}\left[U\left(\xi_{j}, \beta_{j}\right)-U\left(\xi_{j}, \gamma_{j}\right)-\int_{\beta_{j}}^{\gamma_{j}} D U(\tau, t)\right]\right\|<\epsilon .
$$

O próximo teorema trata da extensão de Cauchy para a integral de Kurzweil. Grosseiramente falando, a extensão de Cauchy de uma integral trata de estendê-la por integrais que conhecemos como "impróprias" nos casos da integral de Riemann e da integral de Lebesgue. No caso da integral de Kurzweil, as extensões de Cauchy também são integrais de Kurzweil e, portanto, não há "integrais impróprias", pois as integrais de Kurzweil contêm suas extensões de Cauchy. 
Teorema 3.9 (Vide [74, Teorema 1.14]). Se $U:[a, b] \times[a, b] \rightarrow X$ for uma função tal que para todo $c \in[a, b), U$ for integrável em $[a, c]$ e o limite

$$
\lim _{c \rightarrow b-}\left[\int_{a}^{c} D U(\tau, t)-U(b, c)+U(b, b)\right]=I \in X
$$

existir, então a função $U$ será integrável em $[a, b]$ e teremos

$$
\int_{a}^{b} D U(\tau, t)=I
$$

Similarmente, se a função $U$ for integrável em $[c, b]$ para todo $c \in(a, b]$ e o limite

$$
\lim _{c \rightarrow a+}\left[\int_{c}^{b} D U(\tau, t)+U(a, c)-U(a, a)\right]=I \in X
$$

existir, então a função $U$ será integrável sobre $[a, b]$ e teremos

$$
\int_{a}^{b} D U(\tau, t)=I
$$

Corolário 3.10. Considere o par de funções $f:[a, b] \rightarrow \mathbb{R}^{n}$ e $g:[a, b] \rightarrow R$

i) Suponha que a integral de Kurzweil-Henstock-Stieljes $\int_{a}^{t} f(s) d g(s)$ exista para todo $t \in[a, b) e$

$$
\lim _{t \rightarrow b^{-}}\left(\int_{a}^{t} f(s) d g(s)+f(b)(g(b)-g(t))\right)=I .
$$

Então, $\int_{a}^{b} f(s) d g(s)=I$.

ii) Suponha que a integral de Kurzweil-Henstock-Stieljes $\int_{t}^{b} f(s) d g(s)$ exista para todo $t \in(a, b] e$

$$
\lim _{t \rightarrow b^{-}}\left(\int_{t}^{b} f(s) d g(s)+f(a)(g(t)-g(a))\right)=I .
$$

Então, $\int_{a}^{b} f(s) d g(s)=I$.

Se $U:[a, b] \times[a, b] \rightarrow X$ for integrável, não é verdade que sua integral indefinida $\int_{a}^{s} D U(\tau, t), s \in[a, b]$, será sempre contínua. O teorema a seguir descreve como será esta integral indefinida. 
Teorema 3.11 (Vide [74, Teorema 1.16]). Sejam $U:[a, b] \times[a, b] \rightarrow X$ uma função integrável em $[a, b]$ e $c \in[a, b]$. Então

$$
\lim _{s \rightarrow c}\left[\int_{a}^{s} D U(\tau, t)-U(c, s)+U(c, c)\right]=\int_{a}^{c} D U(\tau, t) .
$$

Vale ressaltar que, nas condições enunciadas acima, também vale o seguinte resultado análogo

$$
\lim _{s \rightarrow c}\left[\int_{s}^{b} D U(\tau, t)-U(c, s)+U(c, c)\right]=\int_{c}^{b} D U(\tau, t) .
$$

Para a integral de Kurzweil, também temos um teorema de mudança de variável que enunciaremos abaixo.

Teorema 3.12 (Vide [74, Teorema 1.18]). Suponhamos que $-\infty<c<d<$ $+\infty$ e que $\varphi:[c, d] \rightarrow \mathbb{R}$ seja uma função contínua estritamente monótona em $[c, d]$. Seja $U:[\varphi(c), \varphi(d)] \times[\varphi(c), \varphi(d)] \rightarrow X$ uma função dada. Se uma das integrais

$$
\int_{\varphi(c)}^{\varphi(d)} D U(\tau, t), \quad \int_{c}^{d} D U(\varphi(\sigma), \varphi(s))
$$

existir, então a outra também existirá e teremos a igualdade

$$
\int_{\varphi(c)}^{\varphi(d)} D U(\tau, t)=\int_{c}^{d} D U(\varphi(\sigma), \varphi(s)) .
$$

Cabe mencionar que o teorema acima também será válido quando a função $\varphi$ for apenas contínua e monótona, ou seja, não-decrescente ou nãocrescente, não havendo necessidade de ser estritamente monótona.

Assim como podemos integrar por partes a integral de Riemann, também podemos integrar por partes a integral de Kurzweil, sendo esta mais uma ferramenta desta integral. A seguir, apresentamos este resultado, que é uma generalização para funções a valores em espaço de Banach de [74, Teorema $1.21]$.

Teorema 3.13. Seja $U:[a, b] \times[a, b] \rightarrow X$ uma função dada. Consideremos $U^{*}(\tau, t)=U(t, \tau)$ e $V(t, \tau)=U(\tau, \tau)-U(\tau, t)-U(t, \tau)+U(t, t)$. Se duas das integrais $\int_{a}^{b} D U(\tau, t), \int_{a}^{b} D U^{*}(\tau, t)$ e $\int_{a}^{b} D V(\tau, t)$ existirem, então a terceira também existirá e teremos a igualdade

$$
\int_{a}^{b} D U(\tau, t)+\int_{a}^{b} D U^{*}(\tau, t)=U(b, b)-U(a, a)-\int_{a}^{b} D V(\tau, t) .
$$


O teorema abaixo é uma generalização de [74, Teorema 1.35] cuja prova pode ser encontrada em [63, Teorema 1.13].

Teorema 3.14. Seja $U:[a, b] \times[a, b] \rightarrow X$ uma função integrável. Se $V$ : $[a, b] \times[a, b] \rightarrow \mathbb{R}$ for integrável e se existir uma função calibre $\theta$ em $[a, b]$ tal que

$$
|t-\tau|\|U(\tau, t)-U(\tau, \tau)\| \leqslant(t-\tau)[V(\tau, t)-V(\tau, \tau)]
$$

para todo $t \in(\tau-\theta(\tau), \tau+\theta(\tau))$, então teremos a desigualdade

$$
\left\|\int_{a}^{b} D U(\tau, t)\right\| \leqslant \int_{a}^{b} D V(\tau, t) .
$$

\subsection{Noções básicas de EDOs generalizadas}

Consideremos um conjunto $\Omega=O \times[a, b]$, onde $O$ é um subconjunto aberto do espaço de Banach $X$ e $G: \Omega \rightarrow X$.

Definição 3.15. Uma função $x:[\alpha, \beta] \rightarrow X$ será uma solução da equação diferencial ordinária generalizada

$$
\frac{d x}{d \tau}=D G(x, t)
$$

com condição inicial $x\left(t_{0}\right)=z_{0}$ no intervalo $[\alpha, \beta] \subset \mathbb{R}$, se $t_{0} \in[\alpha, \beta]$, $(x(t), t) \in \Omega$ para todo $t \in[\alpha, \beta]$ e se a igualdade

$$
x(v)-z_{0}=\int_{t_{0}}^{v} D G(x(\tau), t)
$$

for válida para todo $v \in[\alpha, \beta]$, onde a integral deve ser entendida como sendo a integral de Kurzweil definida anteriormente.

É importante perceber que a notação mencionada em (3.4) é apenas simbólica. A letra $D$ indica que (3.4) é uma equação diferencial generalizada. Além disso, cabe ressaltar que mesmo usando o símbolo $d x / d \tau$ em (3.4), não significa necessariamente que a solução tenha uma derivada.

O próximo resultado diz que a equação integral é satisfeita pela solução da EDO generalizada, sempre que exista. 
Proposição 3.16 (Vide [74, Proposição 3.5]). Se uma função $x:[\alpha, \beta] \rightarrow X$ for uma solução da equação diferencial ordinária generalizada (3.4) em $[\alpha, \beta]$, então para todo $\gamma \in[\alpha, \beta]$ fixado, a igualdade

$$
x(s)=x(\gamma)+\int_{\gamma}^{s} D G(x(\tau), t), \quad s \in[\alpha, \beta]
$$

estará satisfeita. Se uma função $x:[\alpha, \beta] \rightarrow X$ satisfizer a equação integral (3.5) para algum $\gamma \in[\alpha, \beta]$, então x será uma solução da equação diferencial ordinária generalizada (3.4).

A seguir, definiremos algumas classes de funções que utilizaremos ao longo deste capítulo.

Definição 3.17. Uma função $f:[a, b] \rightarrow \mathbb{R}$ será dita uma função escada finita, se existir uma divisão finita $a=\beta_{0}<\beta_{1}<\ldots<\beta_{m}=b$ tal que em cada intervalo aberto $\left(\beta_{i-1}, \beta_{i}\right), i=1,2, \ldots, m$, a função é identicamente igual a uma constante $c_{i} \in \mathbb{R}$.

Definição 3.18. Uma função $f:[a, b] \rightarrow \mathbb{R}$ será dita uma função regrada no intervalo $[a, b]$, se os limites laterais $f(s-)$ e $f(s+)$ existirem para todo $s \in(a, b]$ e $s \in[a, b)$ respectivamente. Denotaremos o espaço das funções regradas por $R([a, b], \mathbb{R})$

É um fato conhecido que toda função regrada em $[a, b]$ é limitada neste intervalo, sendo o limite uniforme de funções escada finitas. É claro, também, que toda função de variação limitada em $[a, b]$ é regrada em $[a, b]$ (vide [9]).

Outra definição importante é a classe especial de funções $G: \Omega \mapsto X$ para a qual é possível obter informações mais específicas sobre as soluções da equação diferencial generalizada (3.4).

Suporemos que $h:[a, b] \rightarrow \mathbb{R}$ seja uma função não decrescente definida em $[a, b]$.

Definição 3.19. Diremos que uma função $G: \Omega \rightarrow X$ pertence à classe $\mathcal{F}(\Omega, h)$, se

$$
\left\|G\left(x, t_{2}\right)-G\left(x, t_{1}\right)\right\| \leqslant\left|h\left(t_{2}\right)-h\left(t_{1}\right)\right|
$$

para todo $\left(x, t_{2}\right),\left(x, t_{1}\right) \in \Omega$ e se

$$
\left\|G\left(x, t_{2}\right)-G\left(x, t_{1}\right)-G\left(y, t_{2}\right)+G\left(y, t_{1}\right)\right\| \leqslant\|x-y\||| h\left(t_{2}\right)-h\left(t_{1}\right) \mid
$$

para todo $\left(x, t_{2}\right),\left(x, t_{1}\right),\left(y, t_{2}\right),\left(y, t_{1}\right) \in \Omega$. 
A proposição seguinte descreve as soluções de (3.4) em função de $G$. Em particular, se $G$ for contínua na segunda variável, então a solução $x$ será uma função contínua.

Proposição 3.20 (Vide [74, Proposição 3.6]). Se $x:[\alpha, \beta] \rightarrow X$ for uma solução da equação diferencial generalizada (3.4) em $[\alpha, \beta]$, então

$$
\lim _{s \rightarrow \sigma}[x(s)-G(x(\sigma), s)+G(x(\sigma), \sigma)]=x(\sigma)
$$

para todo $\sigma \in[\alpha, \beta]$.

O lema a seguir é a combinação de dois resultados, vide [74, Lema 3.9, Corolario 3.16].

Lema 3.21. Suponha que $G \in \mathcal{F}(\Omega, h)$. Se $x:[\alpha, \beta] \rightarrow X,[\alpha, \beta] \subset(a, b)$, for uma função regrada (em particular, uma função de variação limitada) em $[\alpha, \beta]$, então existirá a integral

$$
\int_{\alpha}^{\beta} D G(x(\tau), t)
$$

Além disso, para quaisquer $s_{1}, s_{2} \in[\alpha, \beta]$, valerá a desigualdade

$$
\left\|\int_{s_{1}}^{s_{2}} D G(x(\tau), t)\right\| \leqslant\left|h\left(s_{2}\right)-h\left(s_{1}\right)\right| .
$$

O próximo lema implicará que as soluções de (3.4) são de variação limitada, se $G$ satisfizer a condição $\left(\mathcal{F}_{1}\right)$.

Lema 3.22 (Vide [74, Lema 3.10, Corolario 3.11]). Suponhamos que $G: \Omega \rightarrow$ $X$ satisfaça a condição $\left(\mathcal{F}_{1}\right) . S e[\alpha, \beta] \subset[a, b]$ e $x:[\alpha, \beta] \rightarrow X$ for uma solução de (3.4), então valerá a desigualdade

$$
\left\|x\left(s_{1}\right)-x\left(s_{2}\right)\right\| \leqslant\left|h\left(s_{2}\right)-h\left(s_{1}\right)\right|
$$

para quaisquer $s_{1}, s_{2} \in[\alpha, \beta]$.

Além disso, $x$ será de variação limitada em $[\alpha, \beta]$,

$$
\operatorname{Var}_{\alpha}^{\beta} x \leqslant h(\beta)-h(\alpha)<+\infty .
$$

Em particular, todo ponto em $[\alpha, \beta]$ no qual a função $h$ é contínua também é um ponto de continuidade da solução $x:[\alpha, \beta] \rightarrow X$. 
O próximo lema descreve as descontinuidades de uma solução qualquer da EDO generalizada (3.4) sempre que $G$ satisfizer $\left(\mathcal{F}_{1}\right)$. Em particular, o lema a seguir descreve como serão os "saltos" de $x$, quando existirem.

Lema 3.23 (Vide [74, Lema 3.12]). Se $x:[\alpha, \beta] \rightarrow X$ for uma solução de (3.4) e $G: \Omega \rightarrow X$ satisfizer a condição $\left(\mathcal{F}_{1}\right)$, então

$$
\begin{array}{ll}
x(\sigma+)-x(\sigma)=G(x(\sigma), \sigma+)-G(x(\sigma), \sigma), & \sigma \in[\alpha, \beta), \\
x(\sigma)-x(\sigma-)=G(x(\sigma), \sigma)-G(x(\sigma), \sigma-), & \sigma \in(\alpha, \beta] .
\end{array}
$$

Observação 3.24. Em particular o lema anterior estabelece que se $x:[a, b] \rightarrow$ $\mathbb{R}^{n}$ é uma solução da EDO generalizada (3.4) e $G \in \mathcal{F}(\Omega, h)$, então $x$ é uma função regrada.

Chegamos ao final desta seção conhecendo certas hipóteses que $x$ e $G$ devem satisfazer para que a integral

$$
\int_{\alpha}^{\beta} D G(x(\tau), t)
$$

exista. Agora, estamos em condições de investigar propriedades básicas sobre as soluções de (3.4) que são descritas através desta integral.

\subsection{Existência e unicidade de soluções}

Nesta seção, consideremos $\Omega=O \times(a, b)$, onde $(a, b) \subset \mathbb{R}, O=B_{c}=\{x \in$ $X ;\|x\|<c\}$, com $c>0$ e $-\infty<a<b<+\infty, h:[a, b] \rightarrow \mathbb{R}$ é uma função não decrescente e contínua à esquerda e $G: \Omega \rightarrow X$ pertence à classe $\mathcal{F}(\Omega, h)$ descrita anteriormente. Nestas condições, uma solução de (3.4) é uma função de variação limitada, contínua à esquerda, vide Lema 3.22 e pelo Lema 3.23 tem descontinuidades de primeira espécie. Dessa forma, se para algum $t_{0} \in(a, b)$, o valor da solução $x$ de (3.4) for $x\left(t_{0}\right)=\widetilde{x}$, então o limite à direita no ponto $t_{0}$ satisfará

$$
x\left(t_{0}+\right)=x\left(t_{0}\right)+G\left(x\left(t_{0}\right), t_{0}+\right)-G\left(x\left(t_{0}\right), t_{0}\right)=\widetilde{x}+G\left(\widetilde{x}, t_{0}+\right)-G\left(\widetilde{x}, t_{0}\right) .
$$

Como estamos considerando a possibilidade de (3.4) admitir solução descontínua, pode acontecer o fato de que, para algum $\widetilde{x} \in B_{c}$, isto é, para $\operatorname{algum}\left(\widetilde{x}, t_{0}\right) \in \Omega$, o valor

$$
\widetilde{x}+=\widetilde{x}+G\left(\widetilde{x}, t_{0}+\right)-G\left(\widetilde{x}, t_{0}\right)
$$


não pertença a $O$. Assim, para provar o teorema de existência local de uma solução de (3.4), satisfazendo a condição inicial $x\left(t_{0}\right)=\widetilde{x}$, fazemos a seguinte hipótese natural

$$
\widetilde{x}+=\widetilde{x}+G\left(\widetilde{x}, t_{0}+\right)-G\left(\widetilde{x}, t_{0}\right) \in O .
$$

O teorema a seguir trata da existência local e unicidade de soluções de $(3.4)$.

Teorema 3.25 (Vide [28, Teorema 2.15]). Seja $G: \Omega \rightarrow X$ uma função pertencente à classe $F(\Omega, h)$, onde $\Omega=O \times(a, b)$, e a função $h$ é contínua à esquerda e não decrescente em $[a, b]$. Então para todo $\left(\widetilde{x}, t_{0}\right) \in \Omega$ satisfazendo

$$
\widetilde{x}+=\widetilde{x}+G\left(\widetilde{x}, t_{0}-\right)-G\left(\widetilde{x}, t_{0}\right) \in O,
$$

teremos $\left(\widetilde{x}+, t_{0}\right) \in \Omega$ e existirá um $\Delta>0$ tal que, no intervalo $\left[t_{0}-\Delta, t_{0}+\right.$ $\Delta]$, existirá uma única solução $x$ da equação diferencial generalizada (3.4) satisfazendo $x\left(t_{0}\right)=\widetilde{x}$.

A seguir, definiremos o conceito de continuação à direita de uma solução de $(3.4)$

Definição 3.26. Se $x$ for uma solução de (3.4) em um intervalo $\left[t_{0}, t_{1}\right]$ com $\left[t_{0}, t_{1}\right] \subset[a, b]$, diremos que $z$ é uma continuação a direita de $x$, se existir $t_{2}>t_{1}\left(t_{2}<b\right)$ de modo que $z$ esteja definida em $\left[t_{0}, t_{2}\right]$ e se $z$ coincidir com $x$ em $\left[t_{0}, t_{1}\right]$ e satisfizer a equação (3.4) em $\left[t_{0}, t_{2}\right]$.

Para finalizar esta seção, veremos o conceito de uma solução maximal da EDO generalizada (3.4).

Definição 3.27. Diremos que $x:\left[t_{0}, t_{0}+\sigma\right] \rightarrow X, \sigma>0,\left[t_{0}, t_{0}+\sigma\right) \subset[a, b]$, é uma solução maximal de (3.4), com $x\left(t_{0}\right)=u \in O$, se $x$ for uma solução de (3.4) em todo o intervalo $\left[t_{0}, t_{0}+\beta\right], \beta<\sigma$, e não puder ser prolongada ao intervalo $\left[t_{0}, t_{0}+\sigma\right]$.

\subsection{Dependência contínua para EDOGs}

Nesta seção, apresentaremos resultados sobre dependência contínua de soluções de equações diferenciais generalizadas com respeito aos dados iniciais.

Consideraremos $\Omega$ e $h$ como antes. 
Lema 3.28 (Vide [63, Lema 1.29]). Suponhamos que $G_{k}: \Omega \rightarrow X$ pertença à classe $\mathcal{F}(\Omega, h)$, para $k=0,1,2, \ldots$, onde $h$ é não decrescente e contínua à esquerda. Seja $G_{k} \stackrel{k \rightarrow \infty}{\longrightarrow} G_{0}$ em $\mathcal{F}(\Omega, h)$. Seja $\psi_{k} \in R([\alpha, \beta], X), k=$ $1,2, \ldots,[\alpha, \beta] \subset[a, b]$ tal que $\left\|\psi_{k}-\psi_{0}\right\|_{\infty}=\sup _{\alpha \leqslant t \leqslant \beta}\left\|\psi_{k}(t)-\psi_{0}(t)\right\| \stackrel{k \rightarrow \infty}{\longrightarrow} 0$. Então

$$
\left\|\int_{\alpha}^{\beta} D G_{k}\left(\psi_{k}(\tau), s\right)-\int_{\alpha}^{\beta} D G_{0}\left(\psi_{0}(\tau), s\right)\right\| \stackrel{k \rightarrow \infty}{\longrightarrow} 0 .
$$

O corolário abaixo segue imediatamente do lema anterior.

Corolário 3.29 (Vide [63, Corolário 1.30]). Sejam $G_{k} \stackrel{k \rightarrow \infty}{\longrightarrow} G_{0}$ pertencentes à classe $\mathcal{F}(\Omega, h)$, onde $h$ é não decrescente e contínua à esquerda. Seja $\psi_{k} \in$ $B V([\alpha, \beta], X), k=0,1,2, \ldots,[\alpha, \beta] \subset(a, b)$, tal que $\left\|\psi_{k}-\psi_{0}\right\|_{B V} \stackrel{k \rightarrow \infty}{\longrightarrow} 0$. Então

$$
\left\|\int_{\alpha}^{\beta} D G_{k}\left(\psi_{k}(\tau), s\right)-\int_{\alpha}^{\beta} D G_{0}\left(\psi_{0}(\tau), s\right)\right\| \stackrel{k \rightarrow \infty}{\longrightarrow} 0 .
$$

A seguinte proposição é um resultado sobre dependência contínua que generaliza [74, Teorema 8.2] para valores em espaços de Banach. Sua demonstração é completamente diferente da prova apresentada em [74] que usa o Princípio da Escolha de Helly.

Proposição 3.30 (Vide [2, Proposição 4.11]). Suponhamos que $G_{k}: \Omega \rightarrow X$ pertença a classe $\mathcal{F}(\Omega, h)$, para $k=0,1,2, \ldots$, onde $h$ é não decrescente $e$ contínua à esquerda e que

$$
\lim _{k \rightarrow+\infty} G_{k}(x, t)=G_{0}(x, t)
$$

para $(x, t) \in \Omega$. Sejam $x_{k}:[\alpha, \beta] \rightarrow X, k=1,2, \ldots$, soluções da equação diferencial generalizada

$$
\frac{d x}{d \tau}=D G_{k}(x, t)
$$

em $[\alpha, \beta]$ tais que

$$
\lim _{k \rightarrow+\infty} x_{k}(s)=x_{0}(s), \quad s \in[\alpha, \beta],
$$

$e(x(s), s) \in \Omega$ para $s \in[\alpha, \beta]$. Então $x_{0}:[\alpha, \beta] \rightarrow X$ satisfaz as seguintes condições: 
i) $\left\|x_{0}\left(s_{2}\right)-x_{0}\left(s_{1}\right)\right\| \leqslant h\left(s_{2}\right)-h\left(s_{1}\right)$, se $s_{1} \leqslant s_{2}$ e $s_{1}, s_{2} \in[\alpha, \beta]$;

ii) $\lim _{k \rightarrow+\infty} x_{k}(s)=x_{0}(s)$ uniformemente em $[\alpha, \beta]$;

iii) $x_{0}$ é uma solução da equação diferencial generalizada $\frac{d x}{d \tau}=D G_{0}(x, t)$ em $[\alpha, \beta]$. 


\section{Capítulo 4}

\section{Sistemas Periódicos Neutros}

Neste capítulo, estudaremos o comportamento assintótico de uma equação diferencial funcional neutra com coeficientes periódicos, na qual o período e o retardamento estão relacionados racionalmente. Para esta análise, dividiremos este capítulo em cinco seções, nas primeiras duas seções serão apresentadas a teoria espectral para sistemas neutros colocando ênfase, na segunda seção, nos sistemas neutros periódicos. Na terceira seção apresentaremos nossos resultados principais, calculando explicitamente o operador resolvente de um operador monodrômico, para assim obter informações necessárias sobre seu espectro. Assim, na quarta seção, obteremos resultados sobre estimativas precisas para o comportamento assintótico das soluções destas equações, tendo por hipótese o conhecimento da dominância de um expoente característico. Estes resultados em combinação com os resultados sobre a dominância de raízes de equações características dados por Frasson [41], nos permitem obter resultados inéditos que estendem os trabalhos de Frasson \& Verduyn Lunel [42] e do Philos \& Purnaras [69]. Na quinta seção estudaremos outros casos, como por exemplo uma EDFN com parte periódica neutra, mostrando que a técnica desenvolvida ainda é valida num contexto mais geral. Cabe mencionar que os resultados deste capítulo estão contidos em [43].

\subsection{Teoria espectral para sistemas neutros}

Nesta seção, estudaremos propriedades de equações diferenciais funcionais neutras periódicas através do espectro do gerador infinitesimal do semigrupo solução. 
Consideremos a EDFN linear autônoma dada por

$$
\left\{\begin{array}{l}
\frac{d}{d t} N x_{t}=L x_{t}, \quad t \geqslant 0 \\
x_{0}=\varphi, \quad \varphi \in \mathcal{C}
\end{array}\right.
$$

onde $L, N: \mathcal{C} \rightarrow \mathbb{C}^{n}$ são operadores lineares contínuos, dados por

$$
L \varphi=\int_{-r}^{0} d \zeta(\theta) \varphi(\theta), \quad N \varphi=\varphi(0)-\int_{-r}^{0} d \mu(\theta) \varphi(\theta)
$$

onde $\left.\zeta, \mu \in \operatorname{NBV}\left([-r, 0], \mathbb{C}^{n \times n}\right)\right)$ e $\mu$ é contínua em zero. Agora, associamos com (4.1) um semigrupo fortemente contínuo, chamado operador solução, dado pela translação ao longo das soluções de (4.1)

$$
T(t) \varphi=x_{t}(\cdot ; \varphi)
$$

em que $x(\cdot ; \varphi)$ denota a solução de (4.1). O gerador infinitesimal $A$ do semigrupo $T(t)$ é dado por

$$
\left\{\begin{array}{l}
\mathcal{D}(A)=\left\{\varphi \in \mathcal{C} \mid \frac{d \varphi}{d t} \in \mathcal{C}, N \frac{d \varphi}{d \theta}=L \varphi\right\} \\
A \varphi=\frac{d \varphi}{d \theta}
\end{array}\right.
$$

Seja $\lambda \in \sigma(A)$ um autovalor de $A$. O núcleo $\operatorname{ker}(\lambda I-A)$ é chamado o autoespaço em $\lambda$ e sua dimensão $d_{\lambda}$, a multiplicidade geométrica. O autoespaço generalizado $E_{\lambda}$ é o menor subespaço fechado que contém todos os $\operatorname{ker}\left((\lambda I-A)^{j}\right), j=1,2 \ldots$ e sua dimensão $m_{\lambda}$ é chamada multiplicidade algébrica. Sabemos que existe uma estreita relação entre propriedades espectrais do gerador infinitesimal $A$ e a matriz característica $\Delta(z)$, associada com (4.1), dada por

$$
\Delta(z)=z\left[I-\int_{-r}^{0} d \mu(t) e^{z t}\right]-\int_{-r}^{0} d \zeta(t) e^{z t} .
$$

Ver Diekmann et. al. [24] e Kaashoek \& Verduyn Lunel [54]. Em particular, a multiplicidade geométrica $d_{\lambda}$ é igual à dimensão do espaço nulo de $\Delta(z)$ em $\lambda$ e a multiplicidade algébrica $m_{\lambda}$ é igual à multiplicidade de $z=\lambda$ como um zero de det $\Delta(z)$. Além disso, o autoespaço generalizado em $\lambda$ é dado por

$$
E_{\lambda}=\operatorname{ker}\left((\lambda I-A)^{k_{\lambda}}\right)
$$


onde $k_{\lambda}$ é a ordem de $z=\lambda$ como polo de $\Delta(z)^{-1}$. Usando a matriz de cofatores adj $\Delta(z)$ de $\Delta(z)$, temos a seguinte representação

$$
\Delta(z)^{-1}=\frac{1}{\operatorname{det} \Delta(z)} \operatorname{adj} \Delta(z) .
$$

Da representação (4.2), podemos inferir imediatamente que o espectro de $A$ consiste somente de pontos no espectro pontual, e é dado pelos zeros da equação caraterística

$$
\operatorname{det} \Delta(\lambda)=0
$$

ou seja,

$$
\sigma(A)=\{\lambda \in \mathbb{C} \mid \operatorname{det} \Delta(\lambda)=0\} .
$$

Observemos que $\sigma(A)$ esta contido no semiplano esquerdo $\{z \mid \operatorname{Re} z<\lambda\}$ no plano complexo. Para equações com retardamento, isto é, $N \varphi=\varphi(0)$, a função det $\Delta(\lambda)$ tem um número finito de zeros em faixas da forma $S_{\alpha, \beta}=$ $\{z \mid \alpha<\operatorname{Re} z<\beta\}$ onde $\alpha, \beta \in \mathbb{R}$. Contudo, em geral, para equações diferenciais funcionais neutras, $\operatorname{det} \Delta(z)$ pode ter infinitos zeros em alguma faixa vertical $S_{\alpha, \beta}$.

Definição 4.1. Um autovalor $\lambda$ de $A$ é chamado simples se $m_{\lambda}=1$.

Portanto, autovalores simples de $A$ correspondem a raízes simples da equação caracteristica.

Para $k_{\lambda}=1$, em particular se $\lambda$ é simples, é sabido que

$$
E_{\lambda}=\left\{\theta \mapsto e^{\lambda \theta} v \mid \theta \in[-r, 0], v \in \operatorname{ker}(\Delta(\lambda))\right\}
$$

Veja por exemplo Hale \& Verduyn Lunel [45, Capítulo VII]. Em Kaashoek \& Verduyn Lunel [54] e Diekmann et al [24, Seção IV.3] foi desenvolvido um proceso sistemático para construir uma base canônica para $E_{\lambda}$ usando cadeias de Jordan para $\lambda \in \sigma(A)$ genéricos.

Vamos finalizar esta seção com uma estimativa exponencial sobre o subespaço complementar $Q_{\lambda_{d}}$, dado pela decomposição espectral de $\mathcal{C}$, na Seção 1.5, onde $\lambda_{d}$ é dado na definição seguinte.

Definição 4.2. Dizemos que $\lambda_{d}$ é um autovalor dominante de $A$ se existe $\epsilon>0$ tal que se $\lambda$ é outro autovalor de $A$, então $\operatorname{Re} \lambda<\operatorname{Re} \lambda_{d}-\epsilon$.

O seguinte lema mostra a importância de calcular as projeções $P_{\lambda}$ explicitamente. 
Lema 4.3 (Vide [42, Lema 2.1]). Suponha que $\lambda_{d}$ é um autovalor simples $e$ dominante de $A$. Para $\delta>0$ suficientemente pequeno, existe uma constante positiva $K=K(\delta)$ tal que

$$
\left\|T(t)\left(I-P_{\lambda}\right) \varphi\right\| \leqslant K e^{\left(\operatorname{Re} \lambda_{d}-\delta\right) t}\|\varphi\|, \quad t \geqslant 0 .
$$

\subsection{Teoria espectral para sistemas periódicos neu- tros}

Consideremos agora a EDFN linear periódica não-autônoma

$$
\frac{d}{d t} N(t) x_{t}=L(t) x_{t}
$$

onde $N(t)$ e $L(t)$ são famílias de operadores lineares contínuos, contínuas em $t$ e existe $\omega>0$ tal que $N(t+\omega)=N(t), L(t+\omega)=L(t)$ para todo $t$. Além disso, $N(t)$ é atômico em zero (vide Definição 1.3). Seja $s \in \mathbb{R}$ e $T_{N, L}(t, s)$, $t \geqslant s$, o operador solução da equação (4.3), isto é, $T_{N, L}(t, 0) \varphi=x_{t}(0, \varphi)$, para todo $t \geqslant 0$ e $\varphi \in \mathcal{C}$, onde $x$ é a solução de (4.3) satisfazendo $x_{0}=\varphi$.

Um número complexo $\rho$ é dito um multiplicador característico da EDFN (4.3) se $\rho$ é um autovalor de tipo finito do operador monodrómico $\Pi_{N, L}(s):=$ $T_{N, L}(s+\omega, s)$, isto é, é um ponto isolado do espectro de $\Pi_{N, L}(s)$, cujo autoespaço generalizado tem dimensão finita. No Capítulo 1 vimos que, os multiplicadores característicos são independentes de $s$. Além disso para cada multiplicador característico $\rho$ e cada $s \in \mathbb{R}$, existe uma decomposição $\mathcal{C}=E_{s} \oplus Q_{s}$, onde $E_{s}=\operatorname{ker}\left(\Pi_{N, L}(s)-\rho I\right)^{k}, Q_{s}=\operatorname{Im}\left(\Pi_{N, L}(s)-\rho I\right)^{k}$, para $k$ suficientemente grande e $E_{s}$ tem dimensão finita $d$. Se $\Phi_{s}$ é uma base para $E_{s}$, então existem matrizes $B_{s}$ e $C_{s}(t)$, respectivamente $d \times d$ e $n \times d$, sendo $B_{s}$ constante, o espectro de $e^{B_{s} \omega}$ sendo o conjunto $\{\rho\}$, e $C_{s}(t+\omega)=C_{s}(t)$. Então para todo $\phi=\Phi_{s} b \in E_{s}$,

$$
T_{N, L}(t+s, s) \phi=C_{s}(t) e^{B_{s} t} b .
$$

Desta forma, existe uma representação de Floquet no autoespaço generalizado $E_{s}$ do multiplicador característico $\rho$.

A representação dada pelo Teorema 1.15 é válida para a equação (4.3). De fato, para qualquer $s \in \mathbb{R}$, existe um $\Phi^{s}=\left(\phi_{1}^{s}, \ldots, \phi_{n}^{s}\right) \operatorname{com} N(s) \Phi^{s}=I_{n}$, tal que se $\Psi^{s}=I-\Phi^{s} N(s)$, então

$$
T_{N, L}(t, s)=T_{N}(t, s) \Psi^{s}+U(t, s),
$$


onde $U(t, s)$ é compacto para $t \geqslant s$ e $T_{N}(t, s)$ é o operador solução da equação diferença $N(t) y_{t}=0$.

Usando o mesmo tipo de raciocínio como para o caso autônomo, se $e^{a_{N} \omega}$ é o raio espectral de $\Pi_{N}(s)=T_{N}(s+\omega, s)$, pode-se mostrar que qualquer $\rho$ no espectro de $\Pi_{N, L}(s) \operatorname{com}|\rho|>e^{a_{N} \omega}$ é um autovalor normal e, assim, um multiplicador característico da equação (4.3). Além disso, existe somente um número finito de multiplicadores característicos $\rho$ satisfazendo $|\rho| \geqslant e^{a \omega}$ para todo $a>a_{N}$. Neste caso, o espaço $\mathcal{C}$ pode ser decomposto como $\mathcal{C}=E_{s, a} \oplus Q_{s, a}$, onde $E_{s, a}$ e $Q_{s, a}$ são invariantes sob $\Pi_{N}(s)$, o espectro de $\left.\Pi_{N}(s)\right|_{E_{s, a}}$ consiste somente em multiplicadores $\rho$ tais que $|\rho| \geqslant e^{a \omega}$, $a>a_{N}$, e o espectro de $\left.T_{N}(s+\omega, s)\right|_{Q_{s, a}}$ fica contido no disco de centro em 0 e raio $<e^{(a-\epsilon) \omega}$ para algum $\epsilon>0$. Portanto, existe uma constante $K$ tal que

$$
\left|T_{N, L}(t, s)\right|_{Q_{s, a}} \mid \leqslant K e^{(a-\epsilon)(t-s)}, \quad t \geqslant s .
$$

Da mesma forma, existem matrizes $B_{s, a}$ e $C_{s, a}(t)$, sendo $B_{s, a}$ constante e $C_{s, a}(t) \omega$-periódica, tais que

$$
T_{N, L}(t+s, s) \Phi_{s, a}=C_{s, a}(t) e^{B_{s, a} t}, \quad t \in \mathbb{R}
$$

onde $\Phi_{s, a}$ é uma base para $E_{s, a}$ e os únicos autovalores de $e^{B_{s, a} \omega}$ são os multiplicadores característicos $\rho$ da equação (4.3) com $|\rho| \geqslant e^{a \omega}$.

Note que se $\rho=e^{\lambda \omega}$ é um multiplicador característico simples e dominante, então $B_{s, a}$ é identicamente a $\{\rho\}, \Phi_{s, a}=\{\Phi\}$ e $C_{s, a}(t)$ assume uma forma mais simples

$$
e^{\lambda t} C_{s, a}(t)=T_{N, L}(t+s, s) \Phi .
$$

Como os multiplicadores característicos são independentes do tempo inicial $s$, podemos considerar $s=0$ daqui para frente, portanto a projeção espectral sobre $E_{0, a}$ através de $Q_{0, a}$ pode ser descrita usando a representação de Dunford para $\rho \in s\left(T_{N, L}(\omega, 0)\right) \backslash s\left(T_{N}(\omega, 0)\right), P_{\rho}=\operatorname{Res}_{z=\rho}\left(z I-T_{N, L}(\omega, 0)\right)^{-1}$. Então podemos calcular a projeção espectral usando cálculo de resíduos. Em particular, se $\rho$ é um multiplicador característico simples de $T_{N, L}(\omega, 0)$, a projeção espectral sobre o autoespaço unidimensional é dada por

$$
P_{\rho} \varphi=\lim _{z \rightarrow \rho}(z-\rho)\left(z I-T_{N, L}(\omega, 0)\right)^{-1} \varphi
$$

Para terminar esta seção, enunciamos dois resultados sobre o comportamento assintótico de soluções. A prova destes resultados é idêntica às provas 
de resultados apresentados em [42], bastando ressaltar que, enquanto no caso das EDFR autônomas, é automático que todo elemento não nulo do espectro é do tipo finito, e que há sempre um número finito destes cujo módulo ultrapassa qualquer valor, isto deve ser pedido por hipótese no caso neutro não autônomo.

Teorema 4.4 (Vide [42, Seç. 5]). Sejam $\rho_{j}, j=1,2, \ldots$, os multiplicadores característicos não-nulos do operador $T_{N, L}(\omega, 0)$ ordenados por decrescimento de seus valores em módulo e $\varphi \in \mathcal{C}$. Se $\gamma$ é um número real tal que existe um número finito de multiplicadores característicos de $T_{N, L}(\omega, 0)$ cujos módulos ultrapassem $e^{\gamma \omega}$, então existem constantes positivas $\epsilon$ e $K$ tais que para $t \geqslant s$

$$
\left\|x_{t}(0, \varphi)-\sum_{\left|\rho_{n}\right| \geqslant e^{\gamma \omega}} P_{\rho_{n}}(0) x_{t}(0, \varphi)\right\| \leqslant K e^{(\gamma-\epsilon) t}\|\varphi\| .
$$

Teorema 4.5 (Vide [42, Seç. 5]). Seja $\rho_{d}=e^{\lambda_{d} \omega}$ um multiplicador característico simples e dominante de $\Pi_{N, L}$. Se $C_{0, a}(t)$ é como em (4.5), então existem constantes positivas $K$ e $\epsilon$ tais que o comportamento assintótico da solução $x(t ; 0, \varphi)$ é dado por

$$
\left\|e^{-\lambda_{d} t} x_{t}(0, \varphi)-c(\varphi) C_{0, a}(t)\right\| \leqslant K e^{-\epsilon t}\|\varphi\|, \quad t \geqslant 0,
$$

onde $c(\varphi)$ é definido de tal forma que $P_{\rho_{d}}(0) \varphi=c(\varphi) \Phi$.

\subsection{Uma classe de sistemas periódicos neutros}

Consideremos a seguinte equação diferencial funcional linear neutra periódica

$$
\frac{d}{d t}\left[x(t)+\sum_{j=1}^{p} a_{j} x(t-j \omega)\right]=\sum_{j=1}^{p} b_{j}(t) x(t-j \omega)
$$

em que $a_{j} \in \mathbb{C}, \omega>0, b_{j}(\cdot)$ são funções contínuas a valores complexos definidas em $\mathbb{R}$, tais que não todas são nulas, $b_{j}(t)=b_{j}(t+q \omega)$ para todo $j=1, \ldots, p$ onde $q>0$ é um inteiro (seja $q$ o menor inteiro positivo com essa propriedade). Notemos que o período e o retardamento estão relacionados racionalmente.

Definição 4.6. Dizemos que $x$, uma função contínua a valores complexos definida no intervalo $[-p \omega, \infty)$, é solução da $\operatorname{EDFN}(4.6)$ se $x(t)+\sum_{j=1}^{p} a_{j} x(t-$ $j \omega)$ é continuamente diferenciável para $t \geqslant 0$ e $x$ satisfaz (4.6) para todo $t \geqslant 0$. 
Nestas condições, denotamos o espaço de fase por $\mathcal{C}=C([-p \omega, 0], \mathbb{C})$. A $q \omega$-periodicidade dos coeficientes em (4.6) fornece que para cada $s \geqslant 0$ e inteiros positivos $n \geqslant 0, T(s+(n+1) q \omega, s+n q \omega)=T(s+q \omega, s)$. Portanto definimos o operador monodrômico $\Pi: \mathcal{C} \rightarrow \mathcal{C}$ por

$$
\Pi \varphi=T(q \omega, 0) \varphi
$$

Para calcular o resolvente de П usaremos o seguinte lema técnico.

Lema 4.7. Seja $\varphi$ e $\psi$ funções diferenciáveis tais que $\psi$ é solução da equação (4.6) $e$

$$
\varphi(\theta)=z \psi(\theta)-\psi(\theta+q \omega), \quad \theta \in[-p \omega, 0] .
$$

Definamos $\varphi_{j}(\theta)=\varphi(j \omega+\theta), j=-(p-1), \ldots, 0, b_{l}^{j}(\theta)=b_{l}(j \omega+\theta)$ para $j=0, \ldots,(q-1)$, tais que $b_{l}^{0}(\theta)=b_{l}^{q}(\theta)$, e

$$
\psi_{j}(\theta)=\psi(j \omega+\theta), \quad j=-(p-1), \ldots, q-1 .
$$

Então o $(p+q-1)$-vetor coluna $\Psi(\theta)=\left(\psi_{1-p}(\theta), \ldots, \psi_{q-1}(\theta)\right)^{T}$ satisfaz o seguinte sistema de equações diferenciais ordinárias

$$
H(z) \dot{\Psi}(\theta)=M(\theta) \Psi(\theta)+F(1, p)(\dot{\varphi}(\theta)) .
$$

com condição inicial

$$
\Psi(0)=K(z) \Psi(-\omega)-F(q, q+p-1)(\varphi(-\omega))
$$

onde $H(z)=\left(h_{r, c}\right), M(\theta)=\left(m_{r, c}(\theta)\right)$ and $K(z)=\left(k_{r, c}\right)$ são matrices quadradas de ordem $p+q-1$, tais que

1. para $r=1, \ldots, p-1, h_{r, r}=z, h_{r, r+q}=-1$;

2. $\operatorname{para} r=p$,

- Se $p \geqslant q, h_{r, r}=z+a_{q}, h_{r, c}=a_{p+q-c}, c=q, \ldots, p+q-1, c \neq r ;$

- Se $p<q, h_{r, r}=z, h_{r, c}=a_{p+q-c}, c=q, \ldots, p+q-1$;

3. $\operatorname{para} r=p+1, \ldots, p+q-1, c=r-p, \ldots, r-1, h_{r, r}=1, h_{r, c}=a_{r-c}$, $m_{r, c}(\theta)=b_{r-c}^{r-p}(\theta)$;

4. $\operatorname{para} r=1, \ldots, q-1, k_{r, r+1}=1$; 
5. $\operatorname{para} r=q, \ldots, q+p-1, k_{r, r-q+1}=z$.

e $h_{r, c}=m_{r, c}(\theta)=k_{r, c}=0$ em outros casos.

Finalmente a função $F$ é definida por $F(l, k)(\varphi)=\sum_{j=l}^{k} \varphi_{j-l} e_{j}$.

Demonstração. Da equação (4.7) em $\theta=-l \omega, l=1, \ldots, p$ e (4.8) em $\theta=0$ para $j=1-p, \ldots, q-p-1$, derivamos as seguintes condições de fronteira

$$
\begin{aligned}
& \psi_{j}(0)=\psi_{j+1}(-\omega), \quad j=1-p, \ldots, q-p-1, \\
& \psi_{j}(0)=z \psi_{j+1-q}(-\omega)-\varphi_{j+1-q}(-\omega), \quad j=q-p, \ldots q-1,
\end{aligned}
$$

que podem ser escritas na forma matricial

$$
\Psi(0)=K(z) \Psi(-\omega)-F(q, q+p-1)(\varphi(-\omega))
$$

onde a matriz $K(z)$ e a função $F$ são dadas como no lema. De (4.6) e $\theta \in[-\omega, 0]$ segue que

$$
\sum_{l=1}^{p} a_{l} \dot{\psi}_{j-l}(\theta)+\dot{\psi}_{j}(\theta)=\sum_{l=1}^{p} b_{l}^{j}(\theta) \psi_{j-l}(\theta), \quad j=1, \ldots, q-1 .
$$

Derivando (4.7) em $\theta+j \omega, j=1-p, \ldots, 0$, com $\theta \in[-\omega, 0]$, obtemos as seguintes equações

$$
\begin{gathered}
z \dot{\psi}_{j}(\theta)-\dot{\psi}_{q+i}(\theta)=\dot{\varphi}_{j}(\theta), \quad j=1-p, \ldots,-1, \\
z \dot{\psi}_{0}(\theta)+\sum_{l=1}^{p} a_{l} \dot{\psi}_{q-l}(\theta)=\sum_{l=1}^{p} b_{l}^{q}(\theta) \psi_{q-l}(\theta)+\dot{\varphi}_{0}(\theta), \quad p<q \\
\left(z+a_{q}\right) \dot{\psi}_{0}(\theta)+\sum_{\substack{l=1 \\
l \neq q}}^{p} a_{l} \dot{\psi}_{q-l}(\theta)=\sum_{l=1}^{p} b_{l}^{q}(\theta) \psi_{q-l}(\theta)+\dot{\varphi}_{0}(\theta), \quad p \geqslant q .
\end{gathered}
$$

De (4.11)-(4.14), derivamos o sistema de equações diferenciais ordinárias (4.9) restrito às condições iniciais (4.10).

Ilustraremos o lema com um exemplo.

Exemplo 4.8. Consideremos a equação diferencial funcional periódica neutra (4.6) $\operatorname{com} p=2$,

$$
\frac{d}{d t}\left[x(t)+a_{1} x(t-\omega)+a_{2} x(t-2 \omega)\right]=b_{1}(t) x(t-\omega)+b_{2}(t) x(t-2 \omega) .
$$


onde $b(\cdot)$ é uma função contínua $3 \omega$-periódica, isto é, $b(t+3 \omega)=b(t)$, assim $q=3$ no lema.

Sejam $\varphi$ e $\psi$ funções diferenciáveis, tal que $\psi$ solução da Equação (4.15). O operador resolvente de $\Pi$ é dado por $(z I-\Pi)^{-1} \varphi=\psi$. Portanto

$$
\varphi(\theta)=z \psi(\theta)-\Pi \psi(\theta)
$$

Derivando (4.16) em $\theta$, obtemos

$$
\begin{aligned}
\dot{\varphi}(\theta)= & z \dot{\psi}(\theta)-\dot{\psi}(\theta+3 \omega), \\
= & z \dot{\psi}(\theta)+a_{1} \dot{\psi}(\theta+2 \omega)+a_{2} \dot{\psi}(\theta+\omega) \\
& -b_{1}(\theta) \psi(\theta+2 \omega)-b_{2}(\theta) \psi(\theta+\omega), \text { para }-\omega \leqslant \theta \leqslant 0, \\
\dot{\varphi}(\theta-\omega)= & z \dot{\psi}(\theta-\omega)+a_{1} \dot{\psi}(\theta+\omega)+a_{2} \dot{\psi}(\theta) \\
& -b_{1}(\theta+2 \omega) \psi(\theta+\omega)-b_{2}(\theta+2 \omega) \psi(\theta), \text { para }-2 \omega \leqslant \theta \leqslant-\omega .
\end{aligned}
$$

Introduzindo as variáveis auxiliares $\psi_{j}(\theta)=\psi(\theta+j \omega)$, para $j=1,2$

$$
\begin{aligned}
\dot{\psi}_{1}(\theta)=\dot{\psi}(\theta+\omega)= & -a_{1} \dot{\psi}(\theta)-a_{2} \dot{\psi}(\theta-\omega)+b_{1}(\theta+\omega) \psi(\theta) \\
& +b_{2}(\theta+\omega) \psi(\theta-\omega), \\
\dot{\psi_{2}}(\theta)=\dot{\psi}(\theta+2 \omega)= & -a_{1} \dot{\psi}(\theta+\omega)-a_{2} \dot{\psi}(\theta)+b_{1}(\theta+2 \omega) \psi(\theta+\omega) \\
& +b_{2}(\theta+2 \omega) \psi(\theta) .
\end{aligned}
$$

Portanto utilizando as notações dadas no Lema 4.7, $\varphi_{j}(\theta)=\varphi(j \omega+\theta)$, $b_{l}^{j}(\theta)=b_{j}(j \omega+\theta)$, temos o seguinte sistema de equações diferenciais ordinárias

$$
\begin{aligned}
\dot{\psi}_{1}(\theta)+a_{1} \dot{\psi}_{0}(\theta)+a_{2} \dot{\psi}_{-1}(\theta) & =b_{1}^{1}(\theta) \psi_{0}(\theta)+b_{2}^{1}(\theta) \psi_{-1}(\theta) \\
\dot{\psi}_{2}(\theta)+a_{1} \dot{\psi}_{1}(\theta)+a_{2} \dot{\psi}_{0}(\theta) & =b_{1}^{2}(\theta) \psi_{1}(\theta)+b_{2}^{2}(\theta) \psi_{0}(\theta) \\
z \dot{\psi}_{-1}(\theta)+a_{1} \dot{\psi}_{1}(\theta)+a_{2} \dot{\psi}_{0}(\theta) & =b_{1}^{2}(\theta) \psi_{1}(\theta)+b_{2}^{2}(\theta) \psi_{0}(\theta)+\dot{\varphi}_{-1}(\theta), \\
z \dot{\psi}_{0}(\theta)+a_{1} \dot{\psi}_{2}(\theta)+a_{2} \dot{\psi}_{1}(\theta) & =b_{1}^{3}(\theta) \psi_{2}(\theta)+b_{2}^{3}(\theta) \psi_{1}(\theta)+\dot{\varphi}_{0}(\theta),
\end{aligned}
$$

com condições de fronteira, dadas por (4.16) em $\theta=-\omega,-2 \omega$ respectivamente

$$
\begin{gathered}
\varphi(-\omega)=z \psi(-\omega)-\psi(2 \omega)=z \psi_{0}(-\omega)-\psi_{2}(0) \\
\varphi(-2 \omega)=z \psi(-2 \omega)-\psi(\omega)=z \psi_{-1}(-\omega)-\psi_{1}(0) .
\end{gathered}
$$


Além disso, como $\psi_{j}(\theta)=\psi(j \omega+\theta)$, segue

$$
\begin{aligned}
\psi_{0}(0) & =\psi_{1}(-\omega), \\
\psi_{-1}(0) & =\psi_{0}(-\omega) .
\end{aligned}
$$

Portanto temos a seguinte forma matricial para o sistema de EDOs

$$
\begin{aligned}
\left(\begin{array}{cccc}
a_{2} & a_{1} & 1 & 0 \\
0 & a_{2} & a_{1} & 1 \\
z & a_{2} & a_{1} & 0 \\
0 & z & a_{2} & a_{1}
\end{array}\right)\left(\begin{array}{c}
\dot{\psi}_{-1}(\theta) \\
\dot{\psi}_{0}(\theta) \\
\dot{\psi}_{1}(\theta) \\
\dot{\psi}_{2}(\theta)
\end{array}\right) \\
=\left(\begin{array}{cccc}
b_{2}^{1}(\theta) & b_{1}^{1}(\theta) & 0 & 0 \\
0 & b_{2}^{2}(\theta) & b_{1}^{2}(\theta) & 0 \\
0 & b_{2}^{2}(\theta) & b_{1}^{2}(\theta) & 0 \\
0 & 0 & b_{2}^{3}(\theta) & b_{1}^{3}(\theta)
\end{array}\right)\left(\begin{array}{c}
\psi_{-1}(\theta) \\
\psi_{0}(\theta) \\
\psi_{1}(\theta) \\
\psi_{2}(\theta)
\end{array}\right)+\left(\begin{array}{c}
0 \\
0 \\
\dot{\varphi}_{-1}(\theta) \\
\dot{\varphi}_{0}(\theta)
\end{array}\right)
\end{aligned}
$$

com condições iniciais

$$
\left(\begin{array}{c}
\psi_{-1}(0) \\
\psi_{0}(0) \\
\psi_{1}(0) \\
\psi_{2}(0)
\end{array}\right)=\left(\begin{array}{llll}
0 & 1 & 0 & 0 \\
0 & 0 & 1 & 0 \\
z & 0 & 0 & 0 \\
0 & z & 0 & 0
\end{array}\right)\left(\begin{array}{c}
\psi_{-1}(-\omega) \\
\psi_{0}(-\omega) \\
\psi_{1}(-\omega) \\
\psi_{2}(-\omega)
\end{array}\right)+\left(\begin{array}{c}
0 \\
0 \\
-\varphi_{-1}(-\omega) \\
-\varphi_{0}(-\omega)
\end{array}\right) .
$$

Finalmente, considerando como no Lema 4.7 , o vetor $\Psi=\left(\psi_{-1}, \psi_{0}, \psi_{1}, \psi_{2}\right)^{T}$ e as matrizes

$$
\begin{gathered}
H(z)=\left(\begin{array}{cccc}
a_{2} & a_{1} & 1 & 0 \\
0 & a_{2} & a_{1} & 1 \\
z & a_{2} & a_{1} & 0 \\
0 & z & a_{2} & a_{1}
\end{array}\right), \quad M(\theta)=\left(\begin{array}{cccc}
b_{2}^{1}(\theta) & b_{1}^{1}(\theta) & 0 & 0 \\
0 & b_{2}^{2}(\theta) & b_{1}^{2}(\theta) & 0 \\
0 & b_{2}^{2}(\theta) & b_{1}^{2}(\theta) & 0 \\
0 & 0 & b_{2}^{3}(\theta) & b_{1}^{3}(\theta)
\end{array}\right) \\
K(z)=\left(\begin{array}{cccc}
0 & 1 & 0 & 0 \\
0 & 0 & 1 & 0 \\
z & 0 & 0 & 0 \\
0 & z & 0 & 0
\end{array}\right)
\end{gathered}
$$

podemos reescrever o sistema de EDOs, como foi estabelecido no lema,

$$
\begin{aligned}
H(z) \dot{\Psi}(\theta) & =M(\theta) \Psi(\theta)+\dot{\varphi}_{-1}(\theta) e_{3}+\dot{\varphi}_{0}(\theta) e_{4} \\
\Psi(0) & =K(z) \Psi(-\omega)-\varphi_{-1}(-\omega) e_{3}-\varphi_{0}(-\omega) e_{4}
\end{aligned}
$$


A seguir enunciaremos o lema que exibe o cálculo do resolvente do $\Pi \mathrm{e}$ daremos uma caracterização para o espectro.

Lema 4.9. Sejam $H(z), M(\theta), K(z)$ e F estabelecidas como no Lema 4.7 e $\Omega_{s}^{t}$ a matriz fundamental da equação diferencial ordinária $\dot{X}(t)=H(z)^{-1} M(t) X(t)$, isto é,

$$
\Omega_{s}^{t}(z)=\exp \left(\int_{s}^{t} H(z)^{-1} M(\theta) d \theta\right) .
$$

$O$ resolvente $(z I-\Pi)^{-1} \varphi$ do operador monodrômico $\Pi$, para $z \in \mathbb{C}$ tal que $\operatorname{det} H(z) \neq 0$, é dado por

$$
\begin{array}{r}
(z I-\Pi)^{-1} \varphi(\theta+i \omega)=e_{i+p}^{T} \Omega_{-\omega}^{\theta}(z) \Delta(z)^{-1}\left[G_{\varphi, z}(0)+\right. \\
F(q, q+p-1) \varphi(-\omega)] \\
+e_{i+p}^{T} G_{\varphi, z}(\theta)
\end{array}
$$

para $-\omega \leqslant \theta \leqslant 0, i=1-p, \ldots, 0 . A$ matriz característica $\Delta(z)$ é dada por

$$
\Delta(z)=K(z)-\Omega_{-\omega}^{0}(z)
$$

e $G_{\varphi, z}(\theta)$ é a função escalar de $\theta$ definida por

$$
\begin{aligned}
G_{\varphi, z}(\theta)=H(z)^{-1} F & (1, p) \varphi(\theta)-\Omega_{-\omega}^{\theta}(z) H(z)^{-1} F(1, p) \varphi(-\omega) \\
& +\int_{-\omega}^{\theta} \Omega_{s}^{\theta}(z) H(z)^{-1} M(s) H(z)^{-1} F(1, p) \varphi(s) d s .
\end{aligned}
$$

Além disso, vale a seguinte caracterização do espectro de $\Pi$

$$
\sigma(\Pi)=\{z \in \mathbb{C}: \operatorname{det} \Delta(z)=0\} \cup\{z \in \mathbb{C}: \operatorname{det} H(z)=0\} .
$$

Demonstração. Dado $\varphi \in \mathcal{C}$, suponha que $(z I-\Pi)^{-1} \varphi=\psi$, isto é,

$$
\varphi=z \psi-\Pi \psi
$$

Para facilitar a leitura, como é mais fácil trabalhar com equações diferenciais em lugar de equações integrais, suponha num primeiro instante que $\varphi$ and $\psi$ são diferenciáveis. Denotamos por $\psi(t)$, para $t \geqslant-\omega$, a solução da equação (4.6) que coincide com $\psi$ in $[-p \omega, 0]$. Portanto reescrevemos $(4.21)$ como

$$
\varphi(\theta)=z \psi(\theta)-\psi(\theta+q \omega)
$$


Agora, introduzimos as funções auxiliares

$$
\psi_{i}(\theta)=\psi(\theta+j \omega), \quad j=0, \ldots, q-1
$$

e definimos o $(p+q-1)$-vetor coluna $\Psi(\theta)=\left(\psi_{1-p}(\theta), \psi_{2-p}(\theta), \ldots, \psi_{q-1}(\theta)\right)^{T}$. A seguir, aplicamos o Lema 4.7 e derivamos o sistema de equações diferenciais ordinárias

$$
H(z) \dot{\Psi}(\theta)=M(\theta) \Psi(\theta)+F(1, p) \dot{\varphi}(\theta)
$$

restrito às condições de contorno

$$
\Psi(0)=K(z) \Psi(-\omega)-F(q, q+p-1) \varphi(-\omega) .
$$

onde $H(z), M(\theta), K(z)$ e $F$ são estabelecidas no Lema 4.7.

Primeiro trataremos o caso em que $z$ é tal que det $H(z) \neq 0$. O sistema (4.24) pode ser reescrito como um sistema de equações diferenciais ordinárias

$$
\dot{\Psi}(\theta)=H(z)^{-1} M(\theta) \Psi(\theta)+H(z)^{-1} F(1, p) \dot{\varphi}(\theta) .
$$

Seja $\Omega_{s}^{t}(z)$ a matriz fundamental do sistema homogêneo

$$
\dot{\Psi}(\theta)=H(z)^{-1} M(\theta) \Psi(\theta) .
$$

A fórmula da variação das constantes fornece que a solução do sistema (4.26) é dada por

$$
\Psi(\theta)=\Omega_{-\omega}^{\theta}(z) \Psi(-\omega)+G_{\varphi, z}(\theta)
$$

em que

$$
G_{\varphi, z}(\theta)=\int_{-\omega}^{\theta} \Omega_{s}^{\theta}(z) H(z)^{-1} F(1, p) \dot{\varphi}(s) d s .
$$

Note que $\frac{d}{d s} \Omega_{s}^{\theta}(z)=-\Omega_{s}^{\theta}(z) H(z)^{-1} M(s)$. Integrando (4.28) por partes, conseguimos a equação (4.19). Em (4.27), ainda podemos obter $\Psi(-\omega)$. Usando (4.25) e (4.27) para $\theta=0$ obtemos

$$
\Psi(-\omega)=\left(K(z)-\Omega_{-\omega}^{0}(z)\right)^{-1}\left[G_{\varphi, z}(0)+F(q, q+p-1) \varphi(-\omega)\right] .
$$

Finalmente, notando que para $\theta \in[-\omega, 0]$ e $j=1-p, \ldots, 0, \theta+j \omega$ percorre o intervalo $[-p \omega, 0]$ e $\psi(\theta+j \omega)=\psi_{j}(\theta)=e_{j+p}^{T} \Psi(\theta)$, da equação (4.27) e (4.29) conseguimos (4.18). Para concluir a parte importante da prova, observemos que a expressão para $(z I-\Pi)^{-1} \varphi$ em (4.18) define um operador 
linear limitado para $\varphi \in \mathcal{C}$. Portanto, podemos abandonar a suposição de que $\varphi$ é diferenciável, usando o fato que o conjunto das funções diferenciáveis é denso em $\mathcal{C}$.

Resta provar a afirmação (4.20). A fórmula do resolvente na equação (4.18) é singular quando det $\Delta(z)=0$. Portanto falta analisar o resolvente quando $H(z)$ é singular. Suponha que estamos neste caso e que existe uma solução $\Psi(\theta)$ de (4.24). Como as primeiras $p-1$ linhas de $H$ são linearmente independentes, uma das últimas $q$ linhas de $H$ é uma combinação linear das anteriores. Pelas operações padrão de manipulação algébrica de sistemas correspondentes à subtração desta combinação linear a partir daquela linha, podemos derivar uma equação escalar algébrica envolvendo combinações lineares de $\psi_{j}(\theta), b_{j}^{k}(\theta)$ e $\dot{\varphi}_{j}(\theta)$ para alguns índices $j$, mas necessariamente com elementos $\dot{\varphi}_{j}(\theta)$. Como $\varphi \mapsto \dot{\varphi}_{j}$ é um operador não limitado, se pudéssemos resolver $\psi_{j}$, a solução seria um operador não limitado. Portanto o resolvente deixaria de ser um operador limitado e z está no espectro de $\Pi$.

\subsection{Comportamento assintótico}

Antes de explicitar a fórmula para a projeção espectral $P_{\rho}$ de $\Pi$ sobre o autoespaço $E_{\rho}$ de um multiplicador característico simples $\rho$, precisamos do seguinte lema auxiliar.

Lema 4.10. Seja $\Delta(z)$ uma matriz quadrada de ordem $n$ diferenciável em $z \in \mathbb{C}, n \geqslant 2$, e suponha que $\rho$ é um zero simples de $\operatorname{det} \Delta(\cdot)$. Então existem vetores coluna $u, v$ de ordem $n$ tal que adj $\Delta(\rho)=u v^{T}$.

Demonstração. Afirmamos que

$$
\operatorname{dim}(\operatorname{ker} \Delta(\rho))=1 .
$$

Neste caso o posto de $\Delta(\rho)$ é $n-1$ e ao menos um menor de $\Delta(\rho)$ é não nulo, portanto

$$
\operatorname{adj} \Delta(\rho) \neq 0 \text {. }
$$

Lembremos a fórmula de Laplace

$$
\Delta(z) \cdot \operatorname{adj} \Delta(z)=\operatorname{det} \Delta(z) I_{n} .
$$

Tomando $z=\rho$ in (4.32) deduzimos que

$$
\operatorname{Im}(\operatorname{adj} \Delta(\rho)) \subset \operatorname{ker} \Delta(\rho) .
$$


Da equação (4.31) obtemos posto( $\operatorname{adj} \Delta(\rho)) \geqslant 1$ e da equação (4.33) e (4.30) obtemos posto $(\operatorname{adj} \Delta(\rho)) \leqslant 1$. Portanto $\operatorname{rank}(\operatorname{adj} \Delta(\rho))=1$. Tomando $v^{T}$ uma linha não nula de adj $\Delta(\rho)$, todas as outras linhas são múltiplas dessa e assim o lema esta demonstrado.

Para finalizar o lema provaremos (4.30). Suponha por contradição que $\operatorname{dim}(\operatorname{ker} \Delta(\rho))>1$. Então existem ao menos dois vetores coluna linearmente independentes $v_{1}, v_{2} \in \operatorname{ker} \Delta(z)$. A partir de $\left\{v_{1}, v_{2}\right\}$ completamos uma base $\left\{v_{1}, \ldots, v_{n}\right\}$ para $\mathbb{C}^{n}$. Seja $V$ uma matriz quadrada cuja $i$-ésima coluna é $v_{i}$. Sem perda de generalidade podemos supor que $\operatorname{det} V=1$. As colunas de $\Delta(\rho) V$ são dadas por $\Delta(\rho) v_{i}$ e ao menos suas primeiras duas colunas são não nulas.

Como $\rho$ é um zero simples de $\operatorname{det} \Delta(\cdot)$, existe uma função diferenciável $g(z)$ tal que $g(\rho) \neq 0$ e det $\Delta(z)=(z-\rho) g(z)$. Portanto existe $\delta>0$ tal que $\operatorname{det} \Delta(z) \neq 0$ e $\Delta(z) V$ é não singular, para $0<|z-\rho|<\delta$.

Para $z \neq \rho$ definimos a função matricial $N(z)$ obtida de $\Delta(z) V$ porém multiplicando a primeira coluna por $1 /(z-\rho)$. Então $\operatorname{det} N(z)=g(z)$. Como a primeira coluna de $\Delta(z) V$ é diferenciável em $z$ e nula para $z=\rho$, a primeira coluna de $N(z)$ tem uma singularidade removível em $z=\rho$, portanto se definimos $N(\rho)=\lim _{z \rightarrow \rho} N(z)$ então $N(z)$ é diferenciável e obtemos $\operatorname{det} N(\rho)=g(\rho) \neq 0$. A contradição segue do fato de que a segunda coluna é $\Delta(\rho) v_{2}=0$. Portanto provamos a afirmação (4.30) e a prova do lema esta completa.

Usando a representação de Dunford para a projeção espectral $P_{\rho}$ de $\Pi$ sobre o autoespaço generalizado $E_{\rho}$,

$$
P_{\rho}=\operatorname{Res}_{z=\rho}(z I-\Pi)^{-1},
$$

podemos calcular uma fórmula para a projeção espectral de П usando cálculo de resíduos.

Teorema 4.11. Seja $\rho$ um multiplicador característico simples. Então, considerando $\Omega_{-\omega}^{\theta}(\rho)$ dado por (4.17), o autoespaço generalizado $E_{\rho}$ é gerado por $\Phi$ definido por $\Phi(\theta+i \omega)=e_{i+p}^{T} \Omega_{-\omega}^{\theta}(\rho) u$ para $\theta \in[-\omega, 0]$ e $i=1-p, \ldots, 0$, e a projeção espectral $P_{\rho}: \mathcal{C} \rightarrow E_{\rho}$ é dada por

$$
P_{\rho} \varphi(\theta)=c(\varphi) \Phi(\theta)
$$

onde

$$
c(\varphi)=\left[\left.\frac{d}{d z} \operatorname{det} \Delta(z)\right|_{z=\rho}\right]^{-1} v^{T}\left[G_{\varphi, \rho}(0)+F(q, q+p-1) \varphi(-\omega)\right]
$$


e $u, v$ são $n$-vetores coluna tais que adj $\Delta(\rho)=u v^{T}$, como dados pelo Lema 4.10 .

Demonstração. Como $\rho$ é um zero simples de $\operatorname{det} \Delta(\rho)=0$, existe uma função diferenciável $g(z)$, com $g(\rho)=\frac{d}{d z}[\operatorname{det} \Delta(z)]_{z=\rho} \neq 0$, tal que $\operatorname{det} \Delta(z)=(z-$ $\rho) g(z)$. Para $z \neq \rho$ numa vizinhança de $\rho$,

$$
\Delta(z)^{-1}=\frac{1}{\operatorname{det} \Delta(z)} \operatorname{adj} \Delta(z)=\frac{1}{z-\rho} \frac{\operatorname{adj} \Delta(z)}{g(z)} .
$$

Da Equação (4.18) na página 75 e (4.37), o resolvente de $\Pi$ tem um polo simples em $z=\rho$. Da equação (4.34) e usando o Lema 4.10 obtemos

$$
\begin{aligned}
{\left[P_{\rho} \varphi\right](\theta+i \omega)=} & \lim _{z \rightarrow \rho}\left[(z-\rho)(z I-\Pi)^{-1} \varphi\right](\theta+i \omega) \\
=\{ & \left.e_{i+p}^{T} \Omega_{-\omega}^{\theta}(\rho) u\right\} \\
& \quad \times\left(\frac{v^{T}}{\frac{d}{d z}[\operatorname{det} \Delta(z)]_{z=\rho}}\left[G_{\varphi, \rho}(0)+F(q, q+p-1) \varphi(-\omega)\right]\right) .
\end{aligned}
$$

O fator em (4.38) entre chaves é avaliado como uma função escalar e concentra todas as dependências em $\theta$ e $i$, portanto podemos definir $\Phi$ como estabelecido no teorema. O fator na equação (4.38) entre parênteses é avaliada como um função escalar e concentra toda a dependência em $\varphi$, assim chamamos-la por $c(\varphi)$ como em (4.36) e (4.35) segue.

A existência de um multiplicador característico dominante $\rho_{d}$ nos permite dar uma fórmula explícita do comportamento assintótico das soluções.

Teorema 4.12. Se $\rho_{d} \neq 0$ é um multiplicador característico simples e dominante de $\Pi$, então o comportamento assintótico da solução $x(\cdot ; 0, \varphi)$ de (4.6) é dado por

$$
\lim _{t \rightarrow \infty}(\Phi(t))^{-1} x(t ; 0, \varphi)=c(\varphi)
$$

onde $\Phi(\cdot)$ é a solução através de $\Phi$ onde $\Phi$ e c(·) são dados no Teorema 4.11.

Demonstração. Pelo teorema anterior

$$
c(\varphi)=\left[\left.\frac{d}{d z} \operatorname{det} \Delta(z)\right|_{z=\rho}\right]^{-1} v^{T}\left[G_{\varphi, \rho}(0)+F(q, q+p-1) \varphi(-\omega)\right]
$$

e $u, v$ são $n$-vetores coluna tais que adj $\Delta(\rho)=u v^{T}$. 
Se tomamos $x(t)=\Phi(t)$ vem que $x(t)$ é solução de (4.6), e satisfaz a condição inicial $x_{0}=\Phi$. Portanto,

$$
T_{N, L}(t, 0) \Phi(\theta)=\Phi(t+\theta)=e_{i+p}^{T} \Omega_{-\omega}^{t+\theta}(\rho) u, i=1-p, \ldots, 0
$$

e $C_{0, a}(t)$, definido em (4.5), é dado por

$$
C_{0, a}(t)(\theta)=e^{-\lambda_{d}(t+\theta)} \Phi(t+\theta) .
$$

Aplicando o Teorema 4.5 da página 70 , avaliando a função $C_{0, a}(t)$ em $\theta=0$,

$$
\exists K>0, \epsilon>0,\left\|e^{-\lambda_{d} t} x_{t}(0, \varphi)-c(\varphi) C_{0, a}(t)\right\| \leqslant K e^{-\epsilon t}\|\varphi\|, \quad \forall t \geqslant 0 .
$$

Consequentemente,

$$
\lim _{t \rightarrow \infty} e^{-\lambda_{d} t} x_{t}(0, \varphi)-c(\varphi) e^{-\lambda_{d} t} \Phi(t)=0,
$$

e usando o fato que $\Phi(t)$ é invertível, temos

$$
\lim _{t \rightarrow \infty}(\Phi(t))^{-1} x_{t}(0, \varphi)=c(\varphi) .
$$

O que conclui a prova do teorema.

\subsection{Casos estudados}

Nesta seção, estudaremos alguns casos especiais. No primeiro exemplo será estudada uma classe bastante simples de EDFR periódicas e resultados exatos serão estabelecidos. No segundo exemplo calcularemos o comportamento assintótico de soluções de equações diferenciais neutras com retardamento onde a parte neutra não é necessariamente constante. Assim, mostraremos por meio dos exemplo que as técnicas das seções prévias podem ser estendidas para uma classe maior de equações periódicas.

\subsubsection{Uma classe simples de EDFR periódicas}

Consideremos o problema de valor inicial (PVI) de equações diferenciais com retardamento da forma

$$
\begin{gathered}
\dot{x}(t)=a(t) x(t)+b(t) x(t-1), \\
x_{t_{0}}=\varphi,
\end{gathered}
$$


onde $a(t)$ e $b(t)$ são funções reais e contínuas 1-periódicas. Pela mudança de variáveis

$$
y(t)=\exp \left(-\int_{0}^{t} a\left(s+t_{0}\right) d s\right) x\left(t+t_{0}\right)
$$

obtemos que a equação (4.40) é equivalente ao PVI

$$
\begin{gathered}
\dot{y}(t)=b\left(t+t_{0}\right) e^{-A} y(t-1), \\
x_{0}=\hat{\varphi}=\theta \mapsto\left(\int_{\theta}^{0} a\left(s+t_{0}\right) d s\right) \varphi(\theta),
\end{gathered}
$$

onde $A=\int_{-1}^{0} a(s) d s$. Calculando o resolvente como antes e usando a mesma notação, conseguimos

$$
\Omega_{s}^{t}(z)=\exp \left(\frac{e^{-A}}{z} \int_{s}^{t} b\left(\sigma+t_{0}\right) d \sigma\right) .
$$

Colocando $B=\int_{-1}^{0} b(\sigma) d \sigma$ e $D=e^{-A} B$, temos $\Omega_{-1}^{0}(z)=e^{D / z}$ e obtemos a equação característica

$$
\Delta(z)=z-e^{D / z}=0 .
$$

Seja agora $z=e^{\lambda}$ e observando que a exponencial é $2 \pi i$-periódica,

$$
e^{\lambda}=e^{D / e^{\lambda}} \Longleftrightarrow \lambda-2 k \pi i=\frac{D}{e^{\lambda}}=\frac{D}{e^{\lambda-2 k \pi i}},
$$

portanto é suficiente analisar a equação sobre os expoentes de Floquet

$$
\lambda-D e^{-\lambda}=0
$$

Se

$$
D>-e^{-1}
$$

segue de Frasson [41, Teorema 6] que $\rho=e^{w(D)}$ é um zero simples e dominante de $(4.43)$, onde $w(\cdot)$, conhecida como a função $w$ de Lambert, é a função inversa de $x \mapsto x e^{x}$. Em particular, se $B>-e^{-1}$ e $B=-A$ então $\rho=$ $e^{w\left(B e^{B}\right)}=e^{B}$.

Para aplicar o Teorema 4.12 calculamos

$$
\begin{gathered}
\Delta^{\prime}(\rho)=(\rho+D) / \rho \\
G_{\hat{\varphi}, \rho}(0)=\frac{\hat{\varphi}(0)}{\rho}-\hat{\varphi}(-1)+\frac{e^{-A}}{\rho^{2}} \int_{-1}^{0} b\left(\sigma+t_{0}\right) \Omega_{\sigma}^{0}(\rho) \hat{\varphi}(\sigma) d \sigma .
\end{gathered}
$$


Aplicando o Teorema 4.12, supondo a condição (4.44), obtemos o seguinte comportamento assintótico para as soluções de (4.42)

$$
\begin{aligned}
\lim _{t \rightarrow \infty} y(t) \exp \left(\frac{-e^{-A}}{\rho} \int_{0}^{t} b\left(\sigma+t_{0}\right) d \sigma\right) & \\
& =\frac{\rho}{\rho+D}\left(\hat{\varphi}(0)+\frac{e^{-A}}{\rho} \int_{-1}^{0} b\left(\sigma+t_{0}\right) \Omega_{\sigma}^{0}(\rho) \hat{\varphi}(\sigma) d \sigma\right) .
\end{aligned}
$$

Desfazendo a mudança de variáveis (4.41), obtemos que o comportamento assintótico para soluções do PVI (4.40) é dado por

$$
\begin{aligned}
& \lim _{t \rightarrow \infty} x(t) \exp \left(-\int_{t_{0}}^{t}\left[\frac{e^{-A}}{\rho} b(\tau)+a(\tau)\right] d \tau\right) \\
= & \frac{\rho}{\rho+D}\left(\varphi(0)+\frac{e^{-A}}{\rho} \int_{-1}^{0} b\left(s+t_{0}\right) \exp \left[\int_{s+t_{0}}^{t_{0}}\left(\frac{e^{-A}}{\rho} b(\tau)+a(\tau)\right) d \tau\right] \varphi(s) d s\right) .
\end{aligned}
$$

\subsubsection{Uma classe de EDFN com parte neutra periódica}

Consideremos a EDFN com coeficientes periódicos

$$
\frac{d}{d t}(x(t)+a(t) x(t-\omega))=b(t) x(t-\omega)
$$

onde $b$ é uma função contínua com $b(t)=b(t+\omega)$, $a$ é uma função continuamente diferenciável que satisfaz $a(t)=a(t+\omega)$. Gostaríamos de mostrar que as técnicas desenvolvidas nas seções anteriores podem ser adaptadas para uma classe maior de EDFs onde os coeficientes da parte neutra são funções contínuas.

No espírito do Lema 4.9 calculamos o resolvente $(z I-\Pi)^{-1} \varphi=\psi$ do operador monodrômico $\Pi=T(0, \omega)$ e obtemos a EDO

$$
\dot{\psi}(\theta)+\frac{\dot{a}(\theta)-b(\theta)}{z+a(\theta)} \psi(\theta)=\frac{\dot{\varphi}(\theta)}{z+a(\theta)} .
$$

Seguindo o mesmo raciocínio da prova do Lema 4.9, conseguimos

$$
\left[(z I-\Pi)^{-1} \varphi\right](\theta)=\Omega_{-\omega}^{\theta}(z)\left[z-\Omega_{-\omega}^{0}(z)\right]^{-1}\left(\varphi(-w)+G_{z, \varphi}(0)\right)+G_{z, \varphi}(\theta)
$$

onde

$$
\Omega_{s}^{t}(z)=\frac{z+a(s)}{z+a(t)} \exp \left(\int_{s}^{t} \frac{b(\sigma) d \sigma}{z+a(\sigma)}\right)
$$


$\mathrm{e}$

$$
G_{z, \varphi}(t)=\frac{\varphi(t)}{z+a(t)}-\frac{\Omega_{-\omega}^{t}(z)}{z+a(0)} \varphi(-\omega)+\int_{-\omega}^{t} \frac{b(s) \Omega_{s}^{t}(z)}{(z+a(s))^{2}} \varphi(s) d s .
$$

Devido à periodicidade de $a$, a equação característica lê-se como

$$
\Delta(z)=z-\exp \left(\int_{-\omega}^{0} \frac{b(\sigma) d \sigma}{z+a(\sigma)}\right)=0
$$

Suponha que

(H) algum $\rho \in \mathbb{C}$ é uma raiz simples e dominante da equação característica (4.48) e $|a(\theta)|<|\rho|$ para $\theta \in[0, \omega]$.

Em particular, (H) é satisfeita no casos restritos onde

$\left(\mathrm{H}^{\prime}\right) \max |a(\theta)|<1$ e $\left.i\right)$ a é constante e $\int_{-\omega}^{0} b(s)=0$ ou ii) existe uma constante $\alpha$ tal que $b(t)=\alpha \dot{a}(t)$,

quando obtemos que $\int_{-\omega}^{0} \frac{b(\sigma) d \sigma}{z+a(\sigma)}=0$, então (4.48) torna-se $\Delta(z)=z-1=0$, e $\rho=1$ é o único multiplicador Floquet, que é simples e dominante.

Da equação (4.46), sob a hipótese $(\mathrm{H})$ obtemos que o autoespaço generalizado $E_{\rho}$ é gerado por $\Phi(\theta)=\Omega_{-\omega}^{\theta}(\rho)$, dado em (4.47), e $P_{\rho} \varphi=c(\varphi) \Phi$ onde a função escalar $c(\cdot)$ é dada por

$$
c(\varphi)=\frac{1}{1+\rho \int_{-\omega}^{0} \frac{b(\sigma) d \sigma}{(z+a(\sigma))^{2}}}\left(\frac{\varphi(0)+a(0) \varphi(-\omega)}{\rho+a(0)}+\int_{-\omega}^{0} \frac{b(s) \Omega_{s}^{0}(\rho)}{(z+a(s))^{2}} \varphi(s) d s\right) .
$$

Sob a hipótese $(\mathrm{H})$, do Teorema 4.4 o comportamento assintótico da solução $x(t)$ de (4.45) restrita à condição inicial $x_{0}=\varphi$ é dado por

$$
e^{-\gamma t}|x(t)-c(\varphi) \Phi(t)|<N e^{-\delta t}\|\varphi\| \text {. }
$$

onde $\gamma$ é tal que $\rho=e^{\gamma \omega}$ e $\delta, N$ são constantes adequadas. Se $\left(\mathrm{H}^{\prime}\right)$ é satisfeita quando 1 é o único multiplicador Floquet, podemos tomar algum $\delta<\ln (\max |\rho-a(t)|) / \omega$ e $N=N(\delta)$ como uma constante adequada. 


\section{Capítulo 5}

\section{Existência de soluções limitadas para EDFRs não homogêneas}

\subsection{Introdução}

Neste capítulo, mostraremos a existência de soluções limitadas para as EDFRs lineares não autônomas não homogêneas dadas por

$$
\dot{x}(t)=L(t) x_{t}+f(t), \quad t \geqslant s \quad x(t) \in \mathbb{C}^{n}
$$

com condição inicial

$$
x_{s}=\varphi \in X,
$$

onde $X=C\left([-h, 0], \mathbb{C}^{n}\right)$ e $L(t): X \rightarrow \mathbb{C}^{n}$ um operador linear limitado dado, como no Teorema da Representação de Riesz, por

$$
L(t) \varphi=\int_{0}^{h} d_{\theta}[\zeta(t, \theta)] \varphi(-\theta)
$$

e $f: \mathbb{R} \rightarrow \mathbb{C}^{n}$ uma função contínua e limitada.

Para estabelecer o resultado principal deste capítulo usaremos o conceito de dicotomia exponencial que foi introduzido por Perron [67], em que desenvolveu a teoria de estabilidade das soluções de equações diferenciais não autônomas no caso de dimensão finita. Apesar de que naquele trabalho, a condição de dicotomia exponencial não foi estabelecida explicitamente, Perron deu uma condição para a existência de soluções limitadas para equações lineares não homogêneas. A equivalência desta condição com a propriedade 
de dicotomia exponencial foi estabelecida por Maŭzel [62]. Nesse sentido, Coppel obteve muitos resultados que podem ser encontrados em [10-14].

Seguindo a linha de pesquisa que caracteriza a dicotomia exponencial em termos de Teoremas de "tipo Perron" (vide [11,67]), mostraremos que a condição de dicotomia exponencial é suficiente para garantir a existência de soluções limitadas da equação integral abstrata (EIA)

$$
u(t)=T_{0}(t-s) \varphi+\int_{s}^{t} T_{0}^{\odot *}(t-\tau) C(\tau) u(\tau) d \tau, \quad t \geqslant s,
$$

onde $C(t): X \rightarrow X^{\odot *}$, definido sobre espaço de Banach $\mathrm{X}$, $\odot$-reflexivo com respeito ao $\mathcal{C}_{0}$-semigrupo $T_{0}(t)$, é dado por $C(t) \varphi=B(t) \varphi+(f(t), 0)$, com $B(t)$ uma família de operadores lineares limitados dada por

$$
B(t) \varphi=(L(t) \varphi, 0) .
$$

Observemos que a Equação (5.2) é a equação integral abstrata da EDFR não homogênea (5.1). Portanto, se estabelecermos uma correspondência entre soluções de (5.2) e soluções de (5.1), obteremos a existência de soluções limitadas para as EDFR não homogênea.

O procedimento para estabelecer os resultados neste capítulo é o seguinte: Na segunda seção, daremos a definição de sistemas de evolução e discutiremos a teoria de perturbação para esses sistemas. Na terceira seção, mostraremos que o operador solução associado com (5.2) é um sistema de evolução orientado positivamente (vide Definição 5.1). Além disso, estabeleceremos a correspondência biunívoca entre as soluções da EDFR linear não homogênea (5.1) e as soluções da EIA (5.2). Finalmente na quarta seção, provaremos o teorema principal deste capítulo, que estabelece a condição necessária para a existência de soluções limitadas da EDFR não homogênea.

\subsection{Sistemas de evolução e perturbações limitadas}

No estudo de sistemas não autônomos, importa levar em conta não apenas o tempo percorrido desde o instante inicial, mas também o instante inicial. Portanto, trabalharemos com famílias de operadores a dois parâmetros $U(t, s)$, onde $s$ corresponde ao instante inicial.

Definição 5.1. Uma família a dois parâmetros $U=\{U(t, s)\}_{t \geqslant s}$ de operadores lineares limitados sobre um espaço de Banach $X$ é chamada um sistema de evolução orientado positivamente em $X$ sempre que 
i. $U(s, s)=I$ (a identidade),

ii. $U(t, r) U(r, s)=U(t, s), \quad s \leqslant r \leqslant t$.

Definição 5.2. Uma família a dois parâmetros $V=\{V(s, t)\}_{t \geqslant s}$ de operadores lineares limitados sobre um espaço de Banach $X$ é chamada um sistema de evolução orientado negativamente em $X$ sempre que

i. $V(t, t)=I$ (a identidade),

ii. $V(s, r) V(r, t)=V(s, t), \quad s \leqslant r \leqslant t$.

Lema 5.3 (Vide [24, Seç. XII.2]). O adjunto do sistema de evolução orientado positivamente é o sistema de evolução orientado negativamente, em símbolos temos

$$
V(t, s)=U^{*}(t, s)
$$

Definição 5.4. O sistema de evolução orientado positivamente $U$ é chamado fortemente contínuo se para cada $x \in X$ a aplicação $(t, s) \mapsto U(t, s) x$ é contínua.

No Capítulo 2, foi mostrado que as EDFRs autônomas podem ser vistas como perturbações lineares de EDFRs triviais. Considerando uma perturbação $B$, de $X$ em $X^{\odot *}$, derivamos a fórmula da variação das constantes

$$
u(t)=T_{0}(t-s) u(s)+\int_{s}^{t} T_{0}^{\odot *}(t-\tau) B u(\tau) d \tau
$$

que é uma equação integral abstrata envolvendo a noção de integral fraca-*, isto é,

$$
\left\langle\int_{s}^{t} T_{0}^{\odot *}(t-\tau) B u(\tau) d \tau, x^{\odot}\right\rangle=\int_{s}^{t}\left\langle B u(\tau) x, T_{0}^{\odot}(t-\tau) x^{\odot}\right\rangle
$$

para $x^{\odot} \in X^{\odot}$ arbitrário. Na verdade, foi considerado $X$ um espaço de Banach $\odot$-reflexivo com respeito ao $\mathcal{C}_{0}$-semigrupo $T_{0}(t)$, que é gerado por $A_{0}$, o qual foi perturbado pelo operador linear limitado $B$.

Neste capítulo, trabalhando com EDFRs lineares não autônomas, perturbaremos o gerador $A_{0}$ por uma família de operadores lineares, $\{B(t)\}_{\alpha \leqslant t \leqslant \omega}$, fortemente contínua, isto é, para cada $\varphi \in X$, a aplicação $t \mapsto B(t) \varphi$ é 
contínua de $[\alpha, \omega]$ em $X^{\odot *}$, onde $\alpha, \omega \in \mathbb{R} \cup\{-\infty,+\infty\}$ e $\alpha<\omega$. Assim obteremos a fórmula da variação das constantes

$$
\tilde{U}(t, s) \varphi=T_{0}(t-s) \varphi+\int_{s}^{t} T_{0}^{\odot *}(t-\tau) B(\tau) \tilde{U}(\tau, s) \varphi d \tau
$$

Observemos que a princípio, a integral em (5.3) toma valores no espaço $X^{\odot *}$, não obstante, podemos mostrar que de fato a integral toma valores no subespaço fechado $X^{\odot \odot}=X$. Dentro deste cenário, argumentos usuais de contração serão aplicados para mostrar que (5.3) admite uma solução única $U(t, s)$. Por dualidade e restrições obteremos semigrupos $\left\{U^{*}(t, s)\right\}$, $\left\{U^{\odot}(t, s)\right\}$ e $\left\{U^{\odot *}(t, s)\right\}$ sobre $X^{*}, X^{\odot}$ e $X^{\odot *}$ respectivamente, sempre que possa ser mostrado que os espaços de continuidade forte não dependam de $B(t)$. Analogamente, o domínio dos geradores fraco-* sobre os espaços "grandes" $X^{*}$ e $X^{\odot *}$ são independentes de $B(t)$.

Em [24, Seç. XII.4] foi mostrado que para a EDFR

$$
\dot{x}(t)=x(t-\tau(t))
$$

onde $\tau(t) \geqslant 0$ é uma função limitada tal que $\dot{\tau}(t)=1$ em algum intervalo, o dual de $U(t, s)$ não deixa $X^{\odot} \subset X^{*}$ invariante.

Precisaremos da seguinte hipótese (vide [24, Hipótese XII.4.2]):

Hipótese A. A função $t \mapsto \zeta(t, \cdot)$ é contínua na norma de NBV, isto é,

$$
\operatorname{Var}_{[0, h]}(\zeta(t, \cdot)-\zeta(\tau, \cdot)) \rightarrow 0, \text { quando } \tau \rightarrow t
$$

Sob esta hopótese, Diekmann et al [24, Teorema XII.4.5] mostram que $X^{\odot}$ é invariante pelo sistema de evolução orientado negativamente $V(t, s)=$ $U^{*}(s, t)$. Tomando duais e restrições $V^{\odot}(s, t)=\left.V(s, t)\right|_{X \odot}$ e $U^{\odot *}(t, s)=$ $\left(V^{\odot}(s, t)\right)^{*}$, temos que $U^{\odot *}$ estende $j U j^{-1}$.

Exemplo 5.5. A Hipótese A está satisfeita para a EDFRs da forma

$$
\dot{x}(t)=\sum_{j=1}^{n} a_{j}(t) x\left(t-t_{j}\right)
$$

onde as funções $a_{j}(t)$ são contínuas. 


\subsection{EDFRs lineares não homogêneas}

Nesta seção, consideramos o sistema linear não homogêneo, não autônomo (5.1) onde $L(t) \varphi=\int_{0}^{h} d_{\theta}[\zeta(t, \theta)] \varphi(-\theta)$, com $\zeta$ denotando uma matriz $n \times n$ cujas entradas pertencem ao conjunto de funções NBV e $f: \mathbb{R} \rightarrow \mathbb{C}^{n}$ é uma função contínua.

Alternativamente usando a notação

$$
\left\langle\zeta(t, \cdot), x_{t}\right\rangle_{n}=\int_{0}^{h} d \zeta(t, \theta) x(t-\theta)
$$

podemos reescrever (5.1) como

$$
\dot{x}(t)=\left\langle\zeta(t, \cdot), x_{t}\right\rangle_{n}+f(t) .
$$

Além disso, suponha a condição inicial

$$
x(s+\theta)=\varphi(\theta), \quad-h \leqslant \theta \leqslant 0
$$

e considere a equação integral abstrata

$$
u(t)=T_{0}(t-s) \varphi+\int_{s}^{t} T_{0}^{\odot *}(t-\tau) C(\tau) u(\tau) d \tau, \quad t \geqslant s
$$

em que $C(t): X \rightarrow X^{\odot *}$ é definida por $C(t) \varphi=B(t) \varphi+(f(t), 0)$ onde $B(t): X \rightarrow X^{\odot *}$ é a família de operadores lineares limitados dada por

$$
B(t) \varphi=\left(\langle\zeta(t, \cdot), \varphi\rangle_{n}, 0\right) .
$$

A seguir, enunciaremos um lema auxiliar, que será usado para provar que a EIA (5.5) associada à EDFR não homogênea, admite uma solução única e define um sistema de evolução orientado positivamente.

Lema 5.6 (Vide [24, Lema XII.2.8]). Considere o conjunto

$$
\Delta=\{(t, s) \mid-\infty \leqslant s \leqslant t \leqslant \infty\}
$$

e $f: \Delta \rightarrow X^{\odot *}$ uma função contínua. Defina $v: \Delta \rightarrow X^{\odot *}$ por

$$
v(t, s)=\int_{s}^{t} T_{0}^{\odot *}(t-\tau) f(\tau, s) d \tau .
$$

Então v é contínua e toma valores em $j(X)=X$. 
O próximo teorema é uma generalização para EDFRs não homogêneas de [24, Teorema III.2.4], seguindo algumas de suas ideias.

Teorema 5.7. A fórmula da variação das constantes

$$
U(t, s) \varphi=T_{0}(t-s) \varphi+\int_{s}^{t} T_{0}^{\odot *}(t-\tau) C(\tau) U(\tau, s) \varphi d \tau
$$

onde $(t, s) \in \Delta$ dado em (5.7) e $\varphi \in X$, define unicamente um sistema de evolução orientado positivamente $U$, fortemente contínuo. Além disso,

$$
\|U(t, s)\| \leqslant M e^{\left(\omega_{0}+M K(t, s)\right)(t-s)}
$$

em que $M$ e $\omega_{0}$ são tais que $\left\|T_{0}(t)\right\| \leqslant M e^{\omega_{0} t} e$

$$
K(t, s)=\sup _{s \leqslant \tau \leqslant t}\|B(\tau)\|+\sup _{s \leqslant \tau \leqslant t}|f(\tau)|
$$

Demonstração. Começaremos fazendo uma prova da existência. Para isso, definamos indutivamente

$$
\left\{\begin{array}{l}
U_{k}(t, s) \varphi=\int_{s}^{t} T_{0}^{\odot *}(t-\tau) C(\tau) U_{k-1}(\tau, s) \varphi d \tau, \quad k \geqslant 1 \\
U_{0}(t, s)=T_{0}(t-s) .
\end{array}\right.
$$

Então, pelo Lema 5.6, sabemos que $U_{k}(t, s)$ aplica $X$ em $X$ e $(t, s) \mapsto U_{k}(t, s)$ é contínua. Agora, segue por indução a seguinte estimativa

$$
\left\|U_{k}(t, s)\right\| \leqslant M e^{w(t-s)} \frac{M^{k}[K(t, s)]^{k}(t-s)^{k}}{k !}
$$

onde $K(t, s)$ é como em (5.10).

Portanto, definamos

$$
U(t, s)=\sum_{k=0}^{\infty} U_{k}(t, s)
$$

e notemos que devido à estimativa na norma do operador, o somatório converge uniformemente em $(t, s)$ sobre intervalos limitados. Assim, $(t, s) \mapsto$ $U(t, s) \varphi$ é contínua para cada $\varphi \in X$. Finalmente, por (5.11)-(5.12), temos 
que $U(t, s)$ satisfaz (5.8). De fato,

$$
\begin{aligned}
U(t, s) \varphi & =\sum_{k=0}^{\infty} U_{k}(t, s)=U_{0}(t, s)+\sum_{k=1}^{\infty} U_{k}(t, s) \\
& =T_{0}(t-s) \varphi+\sum_{k=1}^{\infty} \int_{s}^{t} T_{0}^{\odot *}(t-\tau) C(\tau) U_{k-1}(\tau, s) \varphi d \tau \\
& =T_{0}(t-s) \varphi+\int_{s}^{t} T_{0}^{\odot *}(t-\tau) C(\tau) U(\tau, s) \varphi d \tau
\end{aligned}
$$

Para provarmos a unicidade, consideremos $\{W(t, s)\}_{t \geqslant s}$ uma família de evolução de operadores lineares limitados em $X$, tal que $(t, s) \mapsto W(t, s) \varphi$ é contínua para cada $\varphi \in X$ e a equação

$$
W(t, s) \varphi=T_{0}(t-s) \varphi+\int_{s}^{t} T_{0}^{\odot *}(t-\tau) C(\tau) W(\tau, s) \varphi d \tau
$$

se satisfaz. Subtraindo (5.13) de (5.8), obtemos

$$
U(t, s) \varphi-W(t, s) \varphi=\int_{s}^{t} T_{0}^{\odot *}(t-\tau) C(\tau)[U(\tau, s)-W(\tau, s)] \varphi d \tau .
$$

Portanto,

$$
e^{-w t}\|U(t, s) \varphi-W(t, s) \varphi\| \leqslant M K(t, s) \int_{s}^{t} e^{-w \tau}\|U(\tau, s) \varphi-W(\tau, s) \varphi\| d \tau .
$$

Agora, usando a desigualdade de Gronwall, conseguimos $U(t, s) \varphi=W(t, s) \varphi$ para todo $\varphi \in X$. Assim $U(t, s)=W(t, s)$. Finalmente para provar que $U(t, s)$ é uma família de evolução fortemente contínua, primeiro notemos que $U(s, s) \varphi=T_{0}(0) \varphi=\varphi$, portanto $U(s, s)=I$. A segunda propriedade de semigrupo é provada usando (5.8) em $(t, r)$ e substituindo $\varphi$ por $U(r, s) \varphi$. Portanto, note que (5.13) está satisfeita para $W(t, s)=U(t, r) U(r, s)$, em consequência $U(t, s)=U(t, r) U(r, s)$ para $s \leqslant r \leqslant t$. Por fim, como $T_{0}(t)$ é um $\mathcal{C}_{0}$-semigrupo, de (5.8) e do Lema 5.6, temos que $(t, s) \mapsto U(t, s) \varphi$ é contínuo para cada $\varphi \in X$ e portanto $U(t, s)$ é fortemente contínuo.

O seguinte teorema mostra que as soluções da EIA (5.8) estão em correspondência biunívoca com soluções da problema de valor inicial para as EDFRs não homogêneas (5.1). 
Teorema 5.8. Sejam $X, T_{0}(t)$ e $C(t)$ como descritos acima, $U(t, s)$ um sistema de evolução definido pela EIA (5.8). Então $x(t)$ definido por

$$
\begin{array}{r}
x(s+\theta)=\varphi(\theta), \quad-h \leqslant \theta \leqslant 0, \\
x(t)=(U(t, s) \varphi)(0), \quad t \geqslant s
\end{array}
$$

satisfaz (5.1). Reciprocamente, se x é solução da EDFR (5.1) satisfazendo a condição inicial (5.14), então para $t \geqslant s$ e $\theta \in[-h, 0]$,

$$
(U(t, s) \varphi)(\theta)= \begin{cases}\varphi(t-s+\theta), & t+\theta \leqslant s \\ x(t+\theta), & t+\theta \geqslant s .\end{cases}
$$

Para provar o teorema precisaremos de uma variante do Lema 2.30(ii).

Lema 5.9 (Vide [24, Lema XII.3.3]). Seja e e o $i$-ésimo vetor unitário em $\mathbb{C}^{n}$ e seja $r_{i}^{\odot *}=\left(e_{i}, 0\right)$. Para qualquer $\eta \in L_{\mathrm{loc}}^{1}$,

$$
\int_{s}^{t} T_{0}^{\odot *}(t-\tau) \eta(\tau) r_{i}^{\odot *} d \tau=e_{i} \int_{s}^{\max (s, t+\cdot)} \eta(\sigma) d \sigma .
$$

Prova do Teorema 5.8. Fixemos $s \in \mathbb{R}$ e $\varphi \in X$ e definamos as funções contínuas $y$ e $x$ por

$$
\begin{aligned}
& y(t)=\langle\zeta(t, \cdot), U(t, s) \varphi\rangle_{n}+f(t), \quad t \geqslant s, \\
& x(t)=(U(t, s) \varphi)(0), \quad t \geqslant s .
\end{aligned}
$$

Pela definição de $C(t)$,

$$
\begin{aligned}
C(t) U(t, s) \varphi & =B(t) U(t, s) \varphi+(f(t), 0)=\left(\langle\zeta(t, \cdot), U(t, s) \varphi\rangle_{n}, 0\right)+(f(t), 0) \\
& =(y(t), 0) .
\end{aligned}
$$

Lembrando a definição do semigrupo $T_{0}(t)$,

$$
\left(T_{0}(t-s) \varphi\right)(\theta)=\left\{\begin{array}{lc}
\varphi(t+\theta-s), & -h \leqslant t+\theta-s \leqslant 0, \\
\varphi(0), & t+\theta-s \geqslant 0,
\end{array}\right.
$$

consideremos $\theta=0$ na EIA (5.8) e usando (5.15), (5.19), (5.20), obtemos

$$
x(t)=\varphi(0)+\sum_{i=1}^{n} \int_{s}^{t} T_{0}^{\odot *}(\tau-s) y_{i}(\tau) r_{i}^{\odot *} .
$$


Agora, usando o Lema 5.9,

$$
x(t)=\varphi(0)+\sum_{i=1}^{n} e_{i} \int_{s}^{\max (s, t)} y_{i}(\tau) d(\tau)=\varphi(0)+\int_{s}^{t} y(\tau) d(\tau)
$$

e concluímos que $x$ é continuamente diferenciável para $t \geqslant s$ e

$$
\dot{x}(t)=y(t), \quad t \geqslant s .
$$

Por outro lado, a EIA (5.8) para $t \geqslant s$ e $\theta \in[-h, 0]$

$$
(U(t, s) \varphi)(\theta)=\left(T_{0}(t-s) \varphi\right)(\theta)+\left(\int_{s}^{t} T_{0}^{\odot *}(t-\tau) C(\tau) U(\tau, s) \varphi d \tau\right)(\theta)
$$

junto com a definição de $T_{0}(t-s)$ fornece:

1. $\operatorname{para} t+\theta \leqslant s$

$$
(U(t, s) \varphi)(\theta)=\varphi(t-s+\theta)+\int_{s}^{\max (s, t+\theta)} y(\tau) d \tau=\varphi(t-s+\theta),
$$

2. para $t+\theta \geqslant s$

$$
\begin{aligned}
(U(t, s) \varphi)(\theta) & =\varphi(0)+\int_{s}^{\max (s, t+\theta)} y(\tau) d \tau \\
& =\varphi(0)+\int_{s}^{t+\theta} y(\tau) d \tau=x(t+\theta) .
\end{aligned}
$$

Portanto, juntando os dois casos, obtemos

$$
U(t, s) \varphi(\theta)= \begin{cases}\varphi(t-s+\theta), & t+\theta \leqslant s \\ x(t+\theta), & t+\theta \geqslant s\end{cases}
$$

Desde que podemos estender $x$ ao intervalo $[s-h, s]$ pela condição inicial (5.14), podemos reescrever (5.25) como

$$
U(t, s) \varphi(\theta)=x_{t}(\theta)
$$

Finalmente, usando (5.22) e a definição de $y(t)$ em (5.18), obtemos

$$
\dot{x}(t)=\langle\zeta(t, \cdot), U(t, s) \varphi\rangle_{n}+f(t)
$$


e (5.26) implica que

$$
\dot{x}(t)=\left\langle\zeta(t, \cdot), x_{t}\right\rangle_{n}+f(t),
$$

o que mostra a primeira afirmação do teorema.

Reciprocamente, se $x$ é solução da EDFR (5.1) com condição inicial (5.14), a integração de (5.1) proporciona

$$
x(t+\theta)-x(s)=\int_{s}^{t+\theta}\left(\left\langle\zeta(\tau, \cdot), x_{\tau}\right\rangle_{n}+f(\tau)\right) d \tau .
$$

Usando a condição inicial e a definição de $T_{0}(t-s)$, podemos reescrever $(5.27)$ da seguinte forma

$$
x_{t}(\theta)=T_{0}(t-s) \varphi(\theta)+\int_{s}^{\max (s, t+\theta)}\left(\left\langle\zeta(\tau, \cdot), x_{\tau}\right\rangle_{n}+f(\tau)\right) d \tau .
$$

Agora, usando o Lema 5.9 na outra direção e da definição de $C(t)$,

$$
\begin{aligned}
x_{t} & =T_{0}(t-s) \varphi+\sum_{i=1}^{n} \int_{s}^{t} T_{0}^{\odot *}(t-\tau)\left[\left(\left\langle\zeta(\tau, \cdot), x_{\tau}\right\rangle_{n}\right)_{i}+f_{i}(\tau)\right] r_{i}^{\odot *} d \tau \\
& =T_{0}(t-s) \varphi+\int_{s}^{t} T_{0}^{\odot *}(t-\tau) C(\tau) x_{\tau} d \tau
\end{aligned}
$$

A unicidade de soluções da equação (5.8) implica que

$$
U(t, s) \varphi=x_{t},
$$

o que finaliza a prova da segunda afirmação e do teorema.

\subsection{Soluções limitadas para EDFRs não homogêneas}

Consideramos o problema de valor inicial para a EDFR linear homogênea não autônoma,

$$
\left\{\begin{array}{l}
\dot{x}(t)=L(t) x_{t}, \quad t \geqslant s, \quad x(t) \in \mathbb{C}^{n} \\
x_{s}=\varphi
\end{array}\right.
$$

lembrando que $\varphi \in X=C\left([-h, 0], \mathbb{C}^{n}\right)$ e $L(t) \varphi=\int_{0}^{h} d_{\theta}[\zeta(t, \theta)] \varphi(-\theta)$.

$\mathrm{Na}$ sequência daremos a definição de dicotomia exponencial para uma família geral de evolução $\{U(t, s)\}_{t \geqslant s}$. 
Definição 5.10. Uma família de evolução em EDRFs $\{U(t, s)\}_{t \geqslant s}$ tem a propriedade de dicotomia exponencial sobre $\mathbb{R}$ (com constante $\alpha>0$ ) se existem uma constante $M=M(\alpha)>0$ e operadores projeção $P(s): X^{\odot *} \rightarrow X^{\odot *}$, com $s \mapsto P(s) \varphi$ contínua e limitada para cada $\varphi \in X^{\odot *}$, tal que $Q(s)=$ $I-P(s)$ e as seguintes condições se verificam:

1. $P(t) U^{\odot *}(t, s)=U^{\odot *}(t, s) P(s)$;

2. A restrição $\left.U^{\odot *}(t, s)\right|_{\operatorname{Im} Q(s)}$ é invertível como um operador de $\operatorname{Im} Q(s)$ em $\operatorname{Im} Q(t)$ e definamos $U(s, t)$ o operador inverso;

3. $\left\|U^{\odot *}(t, s) P(s)\right\| \leqslant M e^{-\alpha(t-s)}$, para $s \leqslant t$;

4. $\left\|U^{\odot *}(t, s) Q(s)\right\| \leqslant M e^{-\alpha(s-t)}$, para $t \leqslant s$.

Seja $V_{1}$ um subespaço de $X^{\odot *}$ formado pelos valores iniciais de todas as soluções limitadas de equação homogênea (5.28), e seja $V_{2}$ qualquer subespaço fixo de $X$ complementar de $V_{1}$. Temos que $\operatorname{Im} P(s)=V_{1}$ e $\operatorname{ker} P(s)=V_{2}$.

Seja $B C\left(\mathbb{R}, \mathbb{C}^{n}\right)$ o conjunto das funções contínuas e limitadas. Nosso principal objetivo nesta seção é provar o seguinte teorema.

Teorema 5.11. Fixado $s \in \mathbb{R}$. Suponha que o operador solução $\{U(t, s)\}_{t \geqslant s} d a$ EDFR homogênea (5.28) tem a propriedade de dicotomia exponencial. Então existe $r \geqslant 0$ tal que para cada $f \in B C\left(\mathbb{R}, \mathbb{C}^{n}\right)$, a EDFR não homogênea

$$
\dot{x}(t)=L(t) x_{t}+f(t), t \geqslant s
$$

admite uma única solução limitada $x_{t} \operatorname{com} x_{s} \in \operatorname{ker} P(s)$ tal que

$$
\left\|x_{t}\right\| \leqslant r\|f\|
$$

Demonstração. Fixe $f \in B C\left(\mathbb{R}, \mathbb{C}^{n}\right)$. Seja $P(t)$ a função projeção que satisfaz as condições da Definição 5.10. Consideremos

$$
G(t, s)= \begin{cases}P(t) U^{\odot *}(t, s) P(s), & t>s \\ -Q(t) U^{\odot *}(t, s) Q(s), & t<s .\end{cases}
$$

Consideremos $F(t)=(f(t), 0) \in X^{\odot *}$ e definamos o operador $\hat{G}$ por

$$
(\hat{G} F)(t)=\int_{-\infty}^{\infty} G(t, \tau) F(\tau) d \tau
$$


Notemos que a integral acima converge. Com efeito, usando as propriedades 3 e 4 da Definição 5.10 obtemos

$$
\begin{aligned}
\int_{-\infty}^{\infty}\|G(t, s)\| d s & =\int_{-\infty}^{t}\left\|P(t) U^{\odot *}(t, s) P(s)\right\| d s+\int_{t}^{\infty}\left\|Q(t) U^{\odot *}(t, s) Q(s)\right\| d s \\
& \leqslant \int_{-\infty}^{t} M e^{-\alpha(t-s)} d s+\int_{t}^{\infty} M e^{-\alpha(s-t)} d s \leqslant \frac{2 M}{\alpha}
\end{aligned}
$$

Portanto $\hat{G}$ é limitada em $X^{\odot *} \operatorname{com}\|\hat{G} F(t, f)\| \leqslant \frac{2 M}{\alpha}\|f\|_{\infty}$. Agora, consideremos $x_{t}=(\hat{G} F)(t)$. Então, para $t \geqslant s$

$$
\begin{aligned}
& x_{t}-U(t, s) x_{s}=(\hat{G} F)(t)-U(t, s)(\hat{G} F)(s) \\
= & \int_{-\infty}^{t} P(t) U^{\odot *}(t, \tau) P(\tau) F(\tau) d \tau-\int_{t}^{\infty} Q(t) U^{\odot *}(t, \tau) Q(\tau) F(\tau) d \tau \\
& -U(t, s)\left[\int_{-\infty}^{s} P(s) U(s, \tau)^{\odot *} P(\tau) F(\tau) d \tau-\int_{s}^{\infty} Q(s) U(s, \tau)^{\odot *} Q(\tau) F(\tau) d \tau\right] \\
= & \int_{-\infty}^{s} P(t) U^{\odot *}(t, \tau) P(\tau) F(\tau) d \tau+\int_{s}^{t} P(t) U^{\odot *}(t, \tau) P(\tau) F(\tau) d \tau \\
& -\int_{t}^{\infty} Q(t) U^{\odot *}(t, \tau) Q(\tau) F(\tau) d \tau-\int_{-\infty}^{s} U^{\odot *}(t, s) P(s) U(s, \tau)^{\odot *} P(\tau) F(\tau) d \tau \\
& +\int_{s}^{t} U^{\odot *}(t, s) Q(s) U(s, \tau)^{\odot *} Q(\tau) F(\tau) d \tau+\int_{t}^{\infty} Q(t) U^{\odot *}(t, \tau) Q(\tau) F(\tau) d \tau \\
= & \int_{s}^{t} U^{\odot *}(t, \tau) P(\tau) F(\tau) d \tau+\int_{s}^{t} U^{\odot *}(t, \tau) Q(\tau) F(\tau) d \tau \\
= & \int_{s}^{t} U^{\odot *}(t, \tau) F(\tau) d \tau .
\end{aligned}
$$

Portanto,

$$
x_{t}=U(t, s) x_{s}+\int_{s}^{t} U^{\odot *}(t, \tau) F(\tau) d \tau .
$$

Finalmente, para mostrar que $x_{t}$ satisfaz (5.8), observemos que pelo Corolário A.22 segue a seguinte igualdade

$$
\begin{aligned}
\int_{s}^{t} U^{\odot *}(t, \tau) F(\tau) d \tau= & \int_{s}^{t} T_{0}^{\odot *}(t-\tau) F(\tau) d \tau \\
& +\int_{s}^{t} T_{0}^{\odot *}(t-\tau) B(\tau) \int_{s}^{\tau} U^{\odot *}(\tau, \sigma) F(\sigma) d \sigma d \tau
\end{aligned}
$$


Usando a expressão para $U(t, s)$ dada em (5.3) e (5.32) obtemos

$$
\begin{aligned}
x_{t}= & T_{0}(t-s) \varphi+\int_{s}^{t} T_{0}^{\odot *}(\tau) B(\tau) U(\tau, s) \varphi d \tau+\int_{s}^{t} T_{0}^{\odot * *}(t-\tau) F(\tau) d \tau \\
& +\int_{s}^{t} T_{0}^{\odot *}(t-\tau) B(\tau) \int_{s}^{\tau} U^{\odot *}(\tau, \sigma) F(\sigma) d \sigma d \tau \\
= & T_{0}(t-s) \varphi+\int_{s}^{t} T_{0}^{\odot *}(\tau) B(\tau) \overbrace{\left[U(\tau, s) \varphi d \tau+\int_{s}^{\tau} U^{\odot *}(\tau, \sigma) F(\sigma) d \sigma d \tau\right]}^{x_{\tau}} \\
& +\int_{s}^{t} T_{0}^{\odot *}(t-\tau) F(\tau) d \tau \\
= & T_{0}(t-s) \varphi+\int_{s}^{t} T_{0}^{\odot *}(\tau) B(\tau) x_{\tau} d \tau+\int_{s}^{t} T_{0}^{\odot * *}(t-\tau) F(\tau) d \tau
\end{aligned}
$$

Isto é, $x_{t}$ é solução da equação integral

$$
x_{t}=T_{0}(t-s) \varphi+\int_{s}^{t} T_{0}^{\odot *}(t-\tau) C(\tau) x_{\tau} d \tau .
$$

Segue do Teorema 5.8 que $x_{t}$ é solução da EDFR não homogenea (5.1).

Para provarmos a segunda parte do teorema, observemos que do princípio de superposição que, para cada $f \in B C\left(\mathbb{R}, \mathbb{C}^{n}\right)$, a equação não homogênea (5.1) tem uma única solução limitada $x(\cdot) \operatorname{com} x_{s}=\varphi_{f} \in V_{2}$. Consideremos agora o operador linear $S: B C\left(\mathbb{R}, \mathbb{C}^{n}\right) \rightarrow V_{2} \subset X$ que associa $f$ a $\varphi_{f}$ como descrito acima e para $t \geqslant s$ seja $V(t)$ uma família de operadores lineares definido por

$$
\begin{aligned}
V(t): B C\left(\mathbb{R}, \mathbb{C}^{n}\right) & \rightarrow X \\
f & \mapsto x_{t}(S f, f),
\end{aligned}
$$

onde $x$ é a solução de (5.1). Da primeira parte do teorema, a limitação de $x_{t}$ para cada $f \in B C\left(\mathbb{R}, \mathbb{C}^{n}\right)$ nos dá

$$
\sup _{t}|V(t) f|<\infty .
$$

Portanto, pelo Principio de Limitação Uniforme existe uma constante $r \geqslant 0$ tal que $\|V(t)\| \leqslant r$, o que finaliza a prova do teorema. 


\section{Capítulo 6}

\section{EDFNs em medida e EDOs generalizadas}

Neste capítulo, introduziremos uma classe de EDFs, chamadas Equações $D i$ ferenciais Funcionais Neutras em medida, mostraremos que esta classe poderá ser identificada, de maneira biunívoca, com uma certa classe de EDOs generalizadas. Observaremos que essa correspondência entre as soluções de tais classes de equações diferencias será fundamental para a construção dos resultados de existência e unicidade de soluções, assim como a dependência contínua das soluções em relação aos dados iniciais. Para finalizar o capítulo exibiremos um exemplo que ilustra a correspondência.

Os resultados deste capítulo são novos e encontram-se em [31].

\subsection{Introdução}

Seja $h>0$ e $\mathcal{R}=R\left([-h, 0], \mathbb{R}^{n}\right)$ o espaço de Banach das funções regradas munida com a norma do supremo. Consideremos $\Omega \subset \mathcal{R} \times \mathbb{R}$ um subconjunto aberto com elementos $(\sigma, \phi)$. No Capítulo 1, estudamos a teoria geral de equações diferenciais funcionais neutras

$$
\frac{d}{d t}\left[N\left(x_{t}, t\right)\right]=f\left(x_{t}, t\right)
$$

onde $f, N: \Omega \rightarrow \mathbb{R}^{n}$ são aplicações contínuas, com $N$ atômica em zero. Neste capítulo, introduziremos uma classe geral de equações chamadas equações diferenciais funcionais neutras em medida, definidas da forma

$$
D\left[N\left(x_{t}, t\right)\right]=f\left(x_{t}, t\right) D g,
$$


onde $D\left[N\left(x_{t}, t\right)\right]$ e $D g$ são as derivadas distribucionais no sentido de L. Schwartz (vide [18]). Além disso, para $P \subset \mathcal{R}$ um aberto, $f:\left[t_{0}, t_{0}+\sigma\right] \times P \rightarrow$ $\mathbb{R}^{n}$ é uma função regrada e $g:\left[t_{0}, t_{0}+\sigma\right] \rightarrow \mathbb{R}$ é uma função não decrescente.

Estamos interessados no estudo das equações onde a parte neutra é linear não autônoma, portanto consideraremos o operador $N$ da forma $N\left(x_{t}, t\right)=$ $N(t) x_{t}$. Consequentemente, consideremos a equação diferencial funcional

$$
\left\{\begin{array}{l}
D\left[N(t) x_{t}\right]=f\left(x_{t}, t\right) D g \\
x_{t_{0}}=\varphi
\end{array}\right.
$$

que pode ser reescrita na forma integral

$$
\left\{\begin{array}{l}
N(t) x_{t}=N\left(t_{0}\right) \varphi\left(t_{0}\right)+\int_{t_{0}}^{t} f\left(x_{s}, s\right) d g(s) \\
x_{t_{0}}=\varphi
\end{array}\right.
$$

onde a integral do lado direito pode ser entendida no sentido de RiemannStieltjes, Lebesgue-Stieltjes ou ainda Kurzweil.

Em outras palavras, os sistemas (6.2) e (6.3) são equivalentes. Para maiores detalhes sobre esta correspondência veja o trabalho de Das \& Sharma [18].

O operador $N$ ainda satisfaz as condições dadas na seção 1.4, isto é

$$
N(t) \varphi=\varphi(0)-\int_{0}^{h} d_{\theta}[\mu(t, \theta)] \varphi(-\theta)
$$

onde $\mu: \mathbb{R} \times \mathbb{R} \rightarrow \mathbb{R}^{n \times n}$ é uma função mensurável e normalizada satisfazendo

$$
\mu(t, \theta)=0, \theta \leqslant 0 ; \quad \mu(t, \theta)=\mu(t, h), \theta \geqslant h .
$$

Além disso, suponha que $\mu(t, \theta)$ é uma função contínua à direita em $\theta \in(0, h)$, de variação limitada sobre $\theta \in[0, h]$ uniformemente em $t$ e uniformemente não atômica em zero.

Combinando (6.3) e (6.4), obtemos

$$
x(t)-\int_{0}^{h} d_{\theta}[\mu(t, \theta)] x(t-\theta)=x\left(t_{0}\right)-\int_{0}^{h} d_{\theta}\left[\mu\left(t_{0}, \theta\right)\right] \varphi\left(t_{0}-\theta\right)+\int_{t_{0}}^{t} f\left(x_{s}, s\right) d g(s) .
$$

Portanto, a forma integral da equação (6.2), pode ser escrita como

$$
x(t)=x\left(t_{0}\right)+\int_{t_{0}}^{t} f\left(x_{s}, s\right) d g(s)+\int_{0}^{h} d[\mu(t, \theta)] x(t-\theta)-\int_{0}^{h} d\left[\mu\left(t_{0}, \theta\right)\right] \varphi\left(t_{0}-\theta\right) .
$$


Observação 6.1. Vale ressaltar que as equações diferenciais funcionais neutras em medida generalizam as equações diferencias funcionais neutras, apresentadas no livro de Hale \& Verduyn Lunel [45], por exemplo.

Exemplo 6.2. Se $N(t) \varphi=\varphi(0)$ então temos que a equação integral

$$
x(t)=x\left(t_{0}\right)+\int_{t_{0}}^{t} f\left(x_{s}, s\right) d g(s)
$$

representa as chamadas equações diferenciais funcionais com retardamento em medida, introduzidas por Federson, Mesquita \& Slavik [32]. Assim, as EDFN em medida generalizam as EDFR em medida.

\subsection{Correspondência entre EDFNs em medida e EDOs generalizadas}

Nesta seção, estabeleceremos a correspondência entre soluções de EDFNs em medida e soluções de uma classe de EDOs generalizadas. Esta equivalência será essencial para nosso propósito de passar resultados da teoria de EDOs generalizadas para as EDFN em medida.

Sejam $O \subset R\left(\left[t_{0}-h, t_{0}+\sigma\right], \mathbb{R}^{n}\right)$ e $P=\left\{y_{t} \mid y \in O, t \in\left[t_{0}, t_{0}+\sigma\right]\right\} \subset \mathcal{R}$. Na sequência, mostraremos que sob certas condições, a EDFN em medida com forma integral dada por

$y(t)=y\left(t_{0}\right)+\int_{t_{0}}^{t} f\left(y_{s}, s\right) d g(s)+\int_{0}^{h} d_{\theta}[\mu(t, \theta)] y(t-\theta)-\int_{0}^{h} d_{\theta}\left[\mu\left(t_{0}, \theta\right)\right] y\left(t_{0}-\theta\right)$,

onde a solução $y:\left[t_{0}-h, t_{0}+\sigma\right] \rightarrow \mathbb{R}^{n}$ de (6.6) é considerada uma função regrada, pode ser convertida numa EDO generalizada da forma

$$
\frac{d x}{d \tau}=D G(x, t)
$$

onde $x$ toma valores em $O$, isto é, podemos transformar a EDFN em medida cuja solução toma valores em $\mathbb{R}^{n}$, numa EDO generalizada, cuja solução toma valores num espaço de Banach de dimensão infinita.

Introduziremos a notação $[\cdot, \cdot, \cdot]$ onde

$$
[a, b, c]= \begin{cases}b, & b \in[a, c] \\ a, & b \leqslant a \\ c, & b \geqslant c .\end{cases}
$$


O lado direito $G$ da EDO generalizada será dado por

$$
G(x, t)(\vartheta)=F(x, t)(\vartheta)+J(x, t)(\vartheta)
$$

onde para cada $x \in O$ e $t \in\left[t_{0}, t_{0}+\sigma\right]$ as funções $F$ e $J$ são dadas por

$$
\begin{aligned}
& F(x, t)(\vartheta)=\int_{t_{0}}^{\left[t_{0}, \vartheta, t\right]} f\left(x_{s}, s\right) d g(s), \\
& J(x, t)(\vartheta)=\int_{0}^{h} d_{\theta}\left[\mu\left(\left[t_{0}, \vartheta, t\right], \theta\right)\right] x\left(\left[t_{0}, \vartheta, t\right]-\theta\right)-\int_{0}^{h} d_{\theta}\left[\mu\left(t_{0}, \theta\right)\right] x\left(t_{0}-\theta\right) .
\end{aligned}
$$

Como mostraremos a relação entre a solução $x$ de (6.7) e a solução $y$ de (6.6) será descrita por

$$
x(t)(\vartheta)= \begin{cases}y(\vartheta), & \vartheta \in\left[t_{0}-h, t\right], \\ y(t), & \vartheta \in\left[t, t_{0}+\sigma\right],\end{cases}
$$

onde $t \in\left[t_{0}, t_{0}+\sigma\right]$.

As seguintes propriedades serão de muita importância para garantir que se $y \in O$, então $x(t) \in O$ para cada $t \in\left[t_{0}, t_{0}+\sigma\right]$.

Definição 6.3. Seja $O$ um subconjunto de $R\left(\left[t_{0}-h, t_{0}+\sigma\right], \mathbb{R}^{n}\right)$. Dizemos que $O$ tem a propriedade de prolongamento, se para cada $y \in O$ e cada $\bar{t} \in\left[t_{0}-h, t_{0}+\sigma\right]$, a função $\bar{y}$ dada por

$$
\bar{y}(t)= \begin{cases}y(t), & t \in\left[t_{0}-h, \bar{t}\right], \\ y(\bar{t}), & t \in\left(\bar{t}, t_{0}+\sigma\right]\end{cases}
$$

é ainda um elemento de $O$.

Além disso, suponha as seguintes condições em relação à função $f: P \times$ $\left[t_{0}, t_{0}+\sigma\right] \rightarrow \mathbb{R}^{n}:$

(A) A integral $\int_{t_{0}}^{t_{0}+\sigma} f\left(t, y_{t}\right) d g(t)$ existe para cada $y \in O$.

(B) Existe uma função Lebesgue integrável $M:\left[t_{0}, t_{0}+\sigma\right] \rightarrow \mathbb{R}$ em relação a $g$ tal que

$$
\left|\int_{t_{0}}^{t} f(y, s) d g(s)\right| \leqslant \int_{t_{0}}^{t} M(s) d g(s)
$$

para cada $y \in P$ e $t \in\left[t_{0}, t_{0}+\sigma\right]$. 
(C) Existe uma função Lebesgue integrável $L:\left[t_{0}, t_{0}+\sigma\right] \rightarrow \mathbb{R}$ em relação a $g$ tal que

$$
\left|\int_{t_{0}}^{t}[f(y, s)-f(s, z)] d g(s)\right| \leqslant \int_{t_{0}}^{t} L(s)\|y-z\|_{\infty} d g(s)
$$

para cada $y, z \in P$ e para cada $t \in\left[t_{0}, t_{0}+\sigma\right]$.

Para a função normalizada $\mu: \mathbb{R} \times \mathbb{R} \rightarrow \mathbb{R}^{n \times n}$, suporemos a seguinte condição

$\left(\mathrm{A}^{*}\right)$ Existe uma função Lebesgue integrável $C:\left[t_{0}, t_{0}+\sigma\right] \rightarrow \mathbb{R}$ tal que

$$
\begin{aligned}
\mid \int_{0}^{h} d_{\theta}\left[\mu\left(s_{2}, \theta\right)\right] z\left(s_{2}-\theta\right) & -\int_{0}^{h} d_{\theta}\left[\mu\left(s_{1}, \theta\right)\right] z\left(s_{1}-\theta\right) \mid \leqslant \\
& \int_{s_{1}}^{s_{2}} C(s) \int_{0}^{h} d_{\theta}[\mu(s, \theta)]\|z(s-\theta)\| d s
\end{aligned}
$$

para cada $s_{1}, s_{2} \in\left[t_{0}, t_{0}+\sigma\right]$ e $z \in O$.

A seguir, daremos uma propriedade de funções regradas que pode ser encontrada em [32, Lema 3.5].

Lema 6.4. Se y : $\left[t_{0}-h, t_{0}+\sigma\right] \rightarrow \mathbb{R}^{n}$ é uma função regrada, então $s \mapsto\left\|y_{s}\right\|_{\infty}$ é regrada sobre $\left[t_{0}, t_{0}+\sigma\right]$.

Na sequência enunciaremos uma serie de hipóteses que precisaremos para estabelecer os próximos resultados.

(H1) Para $\tilde{x} \in \mathcal{R}$, sejam

$$
O=B_{c}=\left\{z_{t} \in R\left(\left[t_{0}-h, t_{0}+\sigma\right], \mathbb{R}^{n}\right) \mid\left\|z_{t}-\tilde{x}\right\|<c\right\}, \quad c \geqslant 1
$$

com a propriedade do prolongamento e

$$
P_{c}=\left\{y_{t} \mid y \in B_{c}, t \in\left[t_{0}, t_{0}+\sigma\right]\right\} .
$$

(H2) Sejam $g:\left[t_{0}, t_{0}+\sigma\right] \rightarrow \mathbb{R}$ uma função não decrescente e $f: B_{c} \times\left[t_{0}, t_{0}+\right.$ $\sigma] \rightarrow \mathbb{R}^{n}$ satisfazendo as condições (A), (B), (C). Além disso, a função normalizada $\mu: \mathbb{R} \times \mathbb{R} \rightarrow \mathbb{R}^{n \times n}$ satisfaz a condição ( $\left.\mathrm{A}^{*}\right)$.

O próximo teorema nos dará condições suficientes para garantir que a função $G$ dada por (6.8) pertence à classe $\mathcal{F}(\Omega, h)$, introduzida na Definição 3.19 na página 58 , onde $\Omega=B_{c} \times\left[t_{0}, t_{0}+\sigma\right]$. 
Lema 6.5. Considere $B_{c}$ e $P_{c}$ como em (H1) e suponha (H2). Então a função $G: B_{c} \times\left[t_{0}, t_{0}+\sigma\right] \rightarrow R\left(\left[t_{0}-h, t_{0}+\sigma\right], \mathbb{R}^{n}\right)$ dada em (6.8) pertence à classe $\mathcal{F}\left(B_{c} \times\left[t_{0}, t_{0}+\sigma\right], h\right)$, onde $h(t)=h_{1}(t)+h_{2}(t)$ com $h_{1}, h_{2}:\left[t_{0}, t_{0}+\sigma\right] \rightarrow \mathbb{R}$ dadas por

$$
\begin{aligned}
& h_{1}(t)=\int_{t_{0}}^{t}(L(s)+M(s)) d g(s), \\
& h_{2}(t)=\int_{t_{0}}^{t} C(s) \operatorname{Var}_{\theta \in[0, h]} \mu(s, \theta) d s\left(\|\tilde{x}\|_{\infty}+c\right) .
\end{aligned}
$$

Demonstração. A condição (A) implica que as integrais na definição de $F$ existem. Dado $y \in B_{c}$ e $t_{0} \leqslant s_{1}<s_{2} \leqslant t_{0}+\sigma$, temos

$$
F\left(y, s_{2}\right)(\vartheta)-F\left(y, s_{1}\right)(\vartheta)=\int_{s_{1}}^{\left[s_{1}, \vartheta, s_{2}\right]} f\left(y_{s}, s\right) d g(s),
$$

Portanto para $y \in B_{c}$ arbitrário e para $t_{0} \leqslant s_{1}<s_{2} \leqslant t_{0}+\sigma$, usando (B) obtemos

$$
\begin{aligned}
\left\|F\left(y, s_{2}\right)-F\left(y, s_{1}\right)\right\|_{\infty} & =\sup _{t_{0}-r \leqslant \vartheta \leqslant t_{0}+\sigma}\left|F\left(y, s_{2}\right)(\vartheta)-F\left(y, s_{1}\right)(\vartheta)\right| \\
& =\sup _{s_{1} \leqslant \vartheta \leqslant s_{2}}\left|F\left(y, s_{2}\right)(\vartheta)-F\left(y, s_{1}\right)(\vartheta)\right| \\
& =\sup _{s_{1} \leqslant \vartheta \leqslant s_{2}}\left|\int_{s_{1}}^{\vartheta} f\left(y_{s}, s\right) d g(s)\right| \\
& \leqslant \int_{s_{1}}^{s_{2}} M(s) d g(s) \leqslant h_{1}\left(s_{2}\right)-h_{1}\left(s_{1}\right) .
\end{aligned}
$$

Analogamente, usando (C), se $y, z \in B_{c}$ e $t_{0} \leqslant s_{1} \leqslant s_{2} \leqslant t_{0}+\sigma$, então

$$
\begin{aligned}
\| F\left(y, s_{2}\right)-F( & \left.y, s_{1}\right)-F\left(z, s_{2}\right)+F\left(z, s_{1}\right) \|_{\infty} \\
& =\sup _{s_{1} \leqslant \vartheta \leqslant s_{2}}\left|\int_{s_{1}}^{\vartheta}\left[f\left(y_{s}, s\right)-f\left(z_{s}, s\right)\right] d g(s)\right| \\
& \leqslant \sup _{s_{1} \leqslant \vartheta \leqslant s_{2}} \int_{s_{1}}^{\tau} L(s)\left\|y_{s}-z_{s}\right\|_{\infty} d g(s) \\
& \leqslant\|y-z\|_{\infty} \int_{s_{1}}^{s_{2}} L(s) d g(s) \leqslant\|y-z\|_{\infty}\left(h_{1}\left(s_{2}\right)-h_{1}\left(s_{1}\right)\right)
\end{aligned}
$$


(note que a função $s \mapsto\left\|y_{s}-z_{s}\right\|_{\infty}$ é regrada de acordo ao Lema 6.4, e portanto a integral $\int_{s_{1}}^{\vartheta} L(s)\left\|y_{s}-z_{s}\right\|_{\infty} d g(s)$ existe). Assim, segue que $F \in$ $\mathcal{F}\left(B_{c} \times\left[t_{0}, t_{0}+\sigma\right], h_{1}\right)$.

Além disso, dado $x \in B_{c}$ e para $t_{0} \leqslant s_{1}<s_{2}<t_{0}+\sigma$, pela definição da função $J: B_{c} \times\left[t_{0}, t_{0}+\sigma\right] \rightarrow R\left(\left[t_{0}-h, t_{0}+\sigma\right], \mathbb{R}^{n}\right)$ em (6.10), obtemos

$$
\begin{aligned}
& J\left(x, s_{2}\right)(\vartheta)-J\left(x, s_{1}\right)(\vartheta) \\
& =\int_{0}^{h} d_{\theta}\left[\mu\left(\left[s_{1}, \vartheta, s_{2}\right], \theta\right)\right] x\left(\left[s_{1}, \vartheta, s_{2}\right]-\theta\right)-\int_{0}^{h} d_{\theta}\left[\mu\left(s_{1}, \theta\right)\right] x\left(s_{1}-\theta\right),
\end{aligned}
$$

o qual implica

$$
\begin{aligned}
& J\left(x, s_{2}\right)(\vartheta)-J\left(x, s_{1}\right)(\vartheta)-J\left(y, s_{2}\right)(\vartheta)+J\left(y, s_{1}\right)(\vartheta) \\
& =\int_{0}^{h} d_{\theta}\left[\mu\left(\left[s_{1}, \vartheta, s_{2}\right], \theta\right)\right]\left(x\left(\left[s_{1}, \vartheta, s_{2}\right]-\theta\right)-y\left(\left[s_{1}, \vartheta, s_{2}\right]-\theta\right)\right)
\end{aligned}
$$

para $x, y \in B_{c}$ e $t_{0} \leqslant s_{1}<s_{2}<t_{0}+\sigma$.

Portanto, usando (6.11) e a condição ( $\left.\mathrm{A}^{*}\right)$, conseguimos

$$
\begin{aligned}
\left\|J\left(x, s_{2}\right)-J\left(x, s_{1}\right)\right\| & \\
& =\sup _{\vartheta \in\left[t_{0}-h, t_{0}+\sigma\right]}\left|J\left(x, s_{2}\right)(\vartheta)-J\left(x, s_{1}\right)(\vartheta)\right| \\
& =\sup _{\vartheta \in\left[s_{1}, s_{2}\right]}\left|J\left(x, s_{2}\right)(\vartheta)-J\left(x, s_{1}\right)(\vartheta)\right| \\
& =\sup _{\vartheta \in\left[s_{1}, s_{2}\right]}\left|\int_{0}^{h} d_{\theta}[\mu(\vartheta, \theta)] x(\vartheta-\theta)-\int_{0}^{h} d_{\theta}\left[\mu\left(s_{1}, \theta\right)\right] x\left(s_{1}-\theta\right)\right| \\
& \leqslant \int_{s_{1}}^{s_{2}} C(s) \int_{0}^{h} d_{\theta}[\mu(s, \theta)]\|x(s-\theta)\| d s \\
& \leqslant \int_{s_{1}}^{s_{2}} C(s) \int_{0}^{h} d_{\theta}[\mu(s, \theta)] d s\|x\|_{\infty} \\
& \leqslant \int_{s_{1}}^{s_{2}} C(s) \int_{0}^{h} d_{\theta}[\mu(s, \theta)] d s\left(\|\tilde{x}\|_{\infty}+c\right) \leqslant h_{2}\left(s_{2}\right)-h_{2}\left(s_{1}\right) .
\end{aligned}
$$


Analogamente, (6.12) implica

$$
\begin{aligned}
& \left\|J\left(x, s_{2}\right)-J\left(x, s_{1}\right)-J\left(y, s_{2}\right)+J\left(y, s_{1}\right)\right\| \\
& =\sup _{\vartheta \in\left[t_{0}-h, t_{0}+\sigma\right]}\left|J\left(x, s_{2}\right)(\vartheta)-J\left(x, s_{1}\right)(\vartheta)-J\left(y, s_{2}\right)(\vartheta)+J\left(y, s_{1}\right)(\vartheta)\right| \\
& =\sup _{\vartheta \in\left[s_{1}, s_{2}\right]}\left|J\left(x, s_{2}\right)(\vartheta)-J\left(x, s_{1}\right)(\vartheta)-J\left(y, s_{2}\right)(\vartheta)+J\left(y, s_{1}\right)(\vartheta)\right| \\
& \leqslant \sup _{\vartheta \in\left[s_{1}, s_{2}\right]} \mid \int_{0}^{h} d_{\theta}[\mu(\vartheta, \theta)](x(\vartheta-\theta)-y(\vartheta-\theta)) \\
& \quad \quad-\int_{0}^{h} d_{\theta}\left[\mu\left(s_{1}, \theta\right)\right]\left(x\left(s_{1}-\theta\right)-y\left(s_{1}-\theta\right)\right) \mid \\
& \leqslant \int_{s_{1}}^{s_{2}} C(s) \int_{0}^{h} d_{\theta}[\mu(s, \theta)]\|x(s-\theta)-y(s-\theta)\| d s \\
& \leqslant \int_{s_{1}}^{s_{2}} C(s) \operatorname{Var}_{\theta \in[0, h]} \mu(s, \theta)\left\|x_{s}-y_{s}\right\|_{\infty} d s \\
& \leqslant \int_{s_{1}}^{s_{2}} C(s) \operatorname{Var}_{\theta \in[0, h]} \mu(s, \theta) d s\left(\frac{\|x-y\|_{\infty}}{\|\tilde{x}\|_{1}+c}(\|\tilde{x}\|+c)\right) \\
& \leqslant\|x-y\|_{\infty}\left(h_{2}\left(s_{2}\right)-h_{2}\left(s_{1}\right)\right)
\end{aligned}
$$

Portanto, $J \in \mathcal{F}\left(B_{c} \times\left[t_{0}, t_{0}+\sigma\right], h_{2}\right)$. Por fim, usando (6.8) obtemos que $G \in \mathcal{F}\left(B_{c} \times\left[t_{0}, t_{0}+\sigma\right], h\right)$.

A seguinte afirmação é uma versão modificada de [28, Lema 3.3] (que é dedicada a equações diferenciais funcionais com impulso). Neste sentido omitiremos a prova.

Lema 6.6. Considere $B_{c}$ e $P_{c}$ como em (H1). Suponha que $\phi \in P_{c}, g:\left[t_{0}, t_{0}+\right.$ $\sigma] \rightarrow \mathbb{R}$ é uma função não decrescente, e $f: P_{c} \times\left[t_{0}, t_{0}+\sigma\right] \rightarrow \mathbb{R}^{n}$ é tal que a integral $\int_{t_{0}+\sigma}^{t_{0}+\sigma} f\left(y_{t}, t\right) d g(t)$ existe para cada $y \in P_{c}$. Além disso, suponha que $\mu: \mathbb{R} \times \mathbb{R} \rightarrow \mathbb{R}^{n \times n}$ é uma função normalizada que satisfaz a condição $\left(A^{*}\right)$. Consideremos $G$ dada por (6.8) e suponha que $x:\left[t_{0}, t_{0}+\sigma\right] \rightarrow O$ é uma solução de

$$
\frac{d x}{d \tau}=D G(x, t)
$$

com condição inicial $x\left(t_{0}\right)(\vartheta)=\phi(\vartheta)$ para $\vartheta \in\left[t_{0}-h, t_{0}\right]$, e $x\left(t_{0}\right)(\vartheta)=$ $x\left(t_{0}\right)\left(t_{0}\right)$ para $\vartheta \in\left[t_{0}, t_{0}+\sigma\right]$. Se $v \in\left[t_{0}, t_{0}+\sigma\right]$ e $\vartheta \in\left[t_{0}-h, t_{0}+\sigma\right]$, então

$$
\begin{aligned}
& x(v)(\vartheta)=x(v)(v), \quad \vartheta \geqslant v, \\
& x(v)(\vartheta)=x(\vartheta)(\vartheta), \quad v \geqslant \vartheta .
\end{aligned}
$$


A seguir, demonstraremos um teorema que descreve as propriedades da integral Kurzweil-Stieltjes indefinida, este resultado é um caso especial do Teorema 3.11 que será útil na prova dos principais resultados.

Teorema 6.7. Seja $f:[a, b] \rightarrow \mathbb{R}^{n}$ e $g:[a, b] \rightarrow \mathbb{R}$ um par de funções tais que $g$ é regrada e $\int_{a}^{b} f d g$ existe. Então a função

$$
h(t)=\int_{a}^{t} f(s) d g(s), \quad t \in[a, b],
$$

é regrada e satisfaz

$$
\begin{aligned}
& h(t+)=h(t)+f(t) \Delta^{+} g(t), \quad t \in[a, b), \\
& h(t-)=h(t)-f(t) \Delta^{-} g(t), \quad t \in(a, b],
\end{aligned}
$$

onde $\Delta^{+} g(t)=g(t+)-g(t) e \Delta^{-} g(t)=g(t)-g(t-)$.

Demonstração. Usando o fato de que a integral $\int_{a}^{b} f(u) d g(u)$ existe, temos pelo Corolário 3.10, para $t \in[a, b)$

$$
\lim _{s \rightarrow t^{+}}\left[\int_{a}^{s} f(u) d g(u)-f(t) g(s)+f(t) g(t)\right]=\int_{a}^{t} f(u) d g(u),
$$

isto é,

$$
\lim _{s \rightarrow t^{+}}\left[\int_{a}^{s} f(u) d g(u)\right]=\int_{a}^{t} f(u) d g(u)+f(t)\left(g\left(t^{+}\right)-g(t)\right) .
$$

Portanto, para $t \in[a, b)$, obtemos

$$
h\left(t^{+}\right)=h(t)+f(t) \Delta^{+} g(t) .
$$

A outra igualdade é demonstrada de forma análoga.

Os seguintes dois teoremas descrevem a correspondência entre soluções de uma equação diferencial ordinária generalizada e soluções de uma equação diferencial funcional neutra em medida. Estes resultados são novos e estão contidos no artigo [31]. As provas foram inspiradas nos trabalhos [28,32] que descrevem os casos especiais $g(t)=t$ e $N(t) \varphi=\varphi(0)$ respectivamente. 
Teorema 6.8. Considere $B_{c}$ e $P_{c}$ como em (H1) e suponha (H2). Sejam $\phi \in P_{c}$ e $G: B_{c} \times\left[t_{0}, t_{0}+\sigma\right] \rightarrow R\left(\left[t_{0}-h, t_{0}+\sigma\right], \mathbb{R}^{n}\right)$ dado por (6.8) e suponha que $G(x, t) \in R\left(\left[t_{0}-h, t_{0}+\sigma\right], \mathbb{R}^{n}\right)$ para cada $x \in B_{c}, t \in\left[t_{0}, t_{0}+\sigma\right]$. Seja $y \in P_{c}$ uma solução da equação diferencial funcional neutra em medida

$$
\left\{\begin{array}{l}
y(t)=y\left(t_{0}\right)+\int_{t_{0}}^{t} f\left(y_{s}, s\right) d g(s)+\int_{0}^{h} d_{\theta}[\mu(t, \theta)] y(t-\theta)-\int_{0}^{h} d_{\theta}\left[\mu\left(t_{0}, \theta\right)\right] y\left(t_{0}-\theta\right), \\
y_{t_{0}}=\phi .
\end{array}\right.
$$

sobre $\left[t_{0}, t_{0}+\sigma\right]$. Para cada $t \in\left[t_{0}-h, t_{0}+\sigma\right]$, seja

$$
x(t)(\vartheta)= \begin{cases}y(\vartheta), & \vartheta \in\left[t_{0}-h, t\right] \\ y(t), & \vartheta \in\left[t, t_{0}+\sigma\right] .\end{cases}
$$

Então a função $x:\left[t_{0}, t_{0}+\sigma\right] \rightarrow O$ é uma solução da EDO generalizada

$$
\frac{d x}{d \tau}=D G(x, t)
$$

Demonstração. Mostraremos que, para cada $v \in\left[t_{0}, t_{0}+\sigma\right]$, a integral $\int_{t_{0}}^{v} D G(x(\tau), t)$ existe e

$$
x(v)-x\left(t_{0}\right)=\int_{t_{0}}^{v} D G(x(\tau), t) .
$$

Seja $\epsilon>0$ dado. Como $g$ é não decrescente, este pode ter somente um número finito de pontos $t \in\left[t_{0}, v\right]$ tais que $\Delta^{+} g(t) \geqslant \epsilon$; denotemos estes pontos por $t_{1}, \ldots, t_{m}$. Consideremos o calibre $\delta:\left[t_{0}, t_{0}+\sigma\right] \rightarrow \mathbb{R}^{+}$tal que

$$
\begin{gathered}
\delta(\tau)<\min \left\{\frac{t_{k}-t_{k-1}}{2}, k=2, \ldots, m\right\}, \quad \tau \in\left[t_{0}, t_{0}+\sigma\right] ; \\
\delta(\tau)<\min \left\{\left|\tau-t_{k}\right| ; k=1, \ldots, m\right\}, \quad t_{k} \neq \tau \in\left[t_{0}, t_{0}+\sigma\right] .
\end{gathered}
$$

Estas condições asseguram que se um intervalo marcado $(\tau,[c, d])$ é $\delta$-fino, então $[c, d]$ contém, no máximo, um dos pontos $t_{1}, \ldots, t_{m}$. Além disso, $\tau=t_{k}$ sempre que $t_{k} \in[c, d]$.

Como $y_{t_{k}}=x\left(t_{k}\right)_{t_{k}}$, segue do Teorema 6.7 que

$$
\lim _{s \rightarrow t_{k}+} \int_{t_{k}}^{s} L(s)\left\|y_{s}-x\left(t_{k}\right)_{s}\right\|_{\infty} d g(s)=L\left(t_{k}\right)\left\|y_{t_{k}}-x\left(t_{k}\right)_{t_{k}}\right\|_{\infty} \Delta^{+} g\left(t_{k}\right)=0
$$


para cada $k \in\{1, \ldots, m\}$. Assim o calibre $\delta$ pode ser escolhido de tal forma que

$$
\int_{t_{k}}^{t_{k}+\delta\left(t_{k}\right)} L(s)\left\|y_{s}-x\left(t_{k}\right)_{s}\right\|_{\infty} d g(s)<\frac{\epsilon}{4 m+1}, \quad k \in\{1, \ldots, m\}
$$

e também,

$$
\int_{t_{k}}^{t_{k}+\delta\left(t_{k}\right)} C(s) \operatorname{Var}_{\theta \in[0, h]} \mu(s, \theta)\left\|y_{s}-x\left(t_{k}\right)_{s}\right\|_{\infty} d s<\frac{\epsilon}{4 m+1}, \quad k \in\{1, \ldots, m\} .
$$

Usando o Teorema 6.7 de novo, observamos que

$$
\|y(\tau+t)-y(\tau)\| \leqslant h(t+\tau)-h(\tau),
$$

e, portanto,

$$
\|y(\tau+)-y(\tau)\| \leqslant \Delta^{+} h(\tau)<\epsilon, \quad \tau \in\left[t_{0}, t_{0}+\sigma\right] \backslash\left\{t_{1}, \ldots, t_{m}\right\} .
$$

Assim, podemos assumir o calibre $\delta$ tal que

$$
\|y(\rho)-y(\tau)\| \leqslant \epsilon
$$

para cada $\tau \in\left[t_{0}, t_{0}+\sigma\right] \backslash\left\{t_{1}, \ldots, t_{m}\right\}$ e $\rho \in[\tau, \tau+\delta(\tau))$.

Suponha agora que $\left\{\left(\tau_{i},\left[s_{i-1}, s_{i}\right]\right), i=1, \ldots, l\right\}$ é uma partição $\delta$-fina do intervalo $\left[t_{0}, v\right]$. Usando a definição de $x$, pode ser mostrado que

$$
\begin{aligned}
& \left(x\left(s_{i}\right)-x\left(s_{i-1}\right)\right)(\vartheta) \\
& \quad=\int_{s_{i-1}}^{\left[s_{i-1}, \vartheta, s_{i}\right]} f\left(y_{s}, s\right) d g(s)+\int_{0}^{h} d_{\theta}\left[\mu\left(\left[s_{i-1}, \vartheta, s_{i}\right], \theta\right)\right] y\left(\left[s_{i-1}, \vartheta, s_{i}\right]-\theta\right) .
\end{aligned}
$$

De forma análoga, segue da definição de $G$ que

$$
\begin{aligned}
& \left(G\left(x\left(\tau_{i}\right), s_{i}\right)-G\left(x\left(\tau_{i}\right), s_{i-1}\right)\right)(\vartheta) \\
& \quad=\left(F\left(x\left(\tau_{i}\right), s_{i}\right)-F\left(x\left(\tau_{i}\right), s_{i-1}\right)\right)(\vartheta)+\left(J\left(x\left(\tau_{i}\right), s_{i}\right)-J\left(x\left(\tau_{i}\right), s_{i-1}\right)\right)(\vartheta),
\end{aligned}
$$

onde

$$
\begin{aligned}
& \left(F\left(x\left(\tau_{i}\right), s_{i}\right)-F\left(x\left(\tau_{i}\right), s_{i-1}\right)\right)(\vartheta)=\int_{s_{i-1}}^{\left[s_{i-1}, \vartheta, s_{i}\right]} f\left(x\left(\tau_{i}\right)_{s}, s\right) d g(s), \\
& \left(J\left(x\left(\tau_{i}\right), s_{i}\right)-J\left(x\left(\tau_{i}\right), s_{i-1}\right)\right)(\vartheta) \\
& =\int_{0}^{h} d_{\theta}\left[\mu\left(\left[s_{i-1}, \vartheta, s_{i}\right], \theta\right)\right] x\left(\tau_{i}\right)\left(\left[s_{i-1}, \vartheta, s_{i}\right]-\theta\right)-\int_{0}^{h} d_{\theta}\left[\mu\left(s_{i-1}, \theta\right)\right] x\left(\tau_{i}\right)\left(s_{i-1}-\theta\right) .
\end{aligned}
$$


Pela combinação das desigualdades anteriores, obtemos

$$
\begin{aligned}
& \left(x\left(s_{i}\right)-x\left(s_{i-1}\right)\right)(\vartheta)-\left(G\left(x\left(\tau_{i}\right), s_{i}\right)-G\left(x\left(\tau_{i}\right), s_{i-1}\right)\right)(\vartheta) \\
& =\int_{s_{i-1}}^{\left[s_{i-1}, \vartheta, s_{i}\right]}\left(f\left(y_{s}, s\right)-f\left(x\left(\tau_{i}\right)_{s}, s\right)\right) d g(s) \\
& \quad+\int_{0}^{h} d_{\theta}\left[\mu\left(\left[s_{i-1}, \vartheta, s_{i}\right], \theta\right)\right]\left(y\left(\left[s_{i-1}, \vartheta, s_{i}\right]-\theta\right)-x\left(\tau_{i}\right)\left(\left[s_{i-1}, \vartheta, s_{i}\right]-\theta\right)\right) \\
& \quad-\int_{0}^{h} d_{\theta}\left[\mu\left(s_{i-1}, \theta\right)\right]\left(y\left(s_{i-1}-\theta\right)-x\left(\tau_{i}\right)\left(s_{i-1}-\theta\right)\right) .
\end{aligned}
$$

Por conseguinte,

$$
\begin{aligned}
& \quad\left\|x\left(s_{i}\right)-x\left(s_{i-1}\right)-\left(G\left(x\left(\tau_{i}\right), s_{i}\right)-G\left(x\left(\tau_{i}\right), s_{i-1}\right)\right)\right\|_{\infty} \\
& =\sup _{\vartheta \in\left[t_{0}-h, t_{0}+\sigma\right]}\left|\left(x\left(s_{i}\right)-x\left(s_{i-1}\right)\right)(\vartheta)-\left(G\left(x\left(\tau_{i}\right), s_{i}\right)-G\left(x\left(\tau_{i}\right), s_{i-1}\right)\right)(\vartheta)\right| \\
& =\sup _{\vartheta \in\left[s_{i-1}, s_{i}\right]} \mid \int_{s_{i-1}}^{\vartheta}\left(f\left(y_{s}, s\right)-f\left(x\left(\tau_{i}\right)_{s}, s\right)\right) d g(s) \\
& \quad+\int_{0}^{h} d_{\theta}[\mu(\vartheta, \theta)]\left(y(\vartheta-\theta)-x\left(\tau_{i}\right)(\vartheta-\theta)\right) \\
& \quad-\int_{0}^{h} d_{\theta}\left[\mu\left(s_{i-1}, \theta\right)\right]\left(y\left(s_{i-1}-\theta\right)-x\left(\tau_{i}\right)\left(s_{i-1}-\theta\right)\right) \mid \\
& \leqslant \sup _{\vartheta \in\left[s_{i-1}, s_{i}\right]}\left|\int_{s_{i-1}}^{\vartheta}\left(f\left(y_{s}, s\right)-f\left(x\left(\tau_{i}\right)_{s}, s\right)\right) d g(s)\right| \\
& +\sup _{\vartheta \in\left[s_{i-1}, s_{i}\right]} \mid \int_{0}^{h} d_{\theta}[\mu(\vartheta, \theta)]\left(y(\vartheta-\theta)-x\left(\tau_{i}\right)(\vartheta-\theta)\right) \\
& \quad-\int_{0}^{h} d_{\theta}\left[\mu\left(s_{i-1}, \theta\right)\right]\left(y\left(s_{i-1}-\theta\right)-x\left(\tau_{i}\right)\left(s_{i-1}-\theta\right)\right) \mid .
\end{aligned}
$$

Pela definição de $x$, temos que $x\left(\tau_{i}\right)_{s}=y_{s}$ quando $s \leqslant \tau_{i}$. Portanto,

$$
\begin{aligned}
\int_{s_{i-1}}^{\vartheta}\left(f\left(y_{s}, s\right)-f\left(x\left(\tau_{i}\right)_{s}, s\right)\right) d g(s) & \\
& = \begin{cases}0, & \vartheta \in\left[s_{i-1}, \tau_{i}\right], \\
\int_{\tau_{i}}^{\vartheta}\left(f\left(y_{s}, s\right)-f\left(x\left(\tau_{i}\right)_{s}, s\right)\right) d g(s), & \vartheta \in\left[\tau_{i}, s_{i}\right] .\end{cases}
\end{aligned}
$$


Então, pela condição (C), obtemos

$$
\begin{aligned}
\left|\int_{\tau_{i}}^{\vartheta}\left(f\left(y_{s}, s\right)-f\left(x\left(\tau_{i}\right)_{s}, s\right)\right) d g(s)\right| & \leqslant \int_{\tau_{i}}^{\vartheta} L(s)\left\|y_{s}-x\left(\tau_{i}\right)_{s}\right\|_{\infty} d g(s) \\
& \leqslant \int_{\tau_{i}}^{s_{i}} L(s)\left\|y_{s}-x\left(\tau_{i}\right)_{s}\right\|_{\infty} d g(s)
\end{aligned}
$$

e analogamente, a condição $\left(\mathrm{A}^{*}\right)$ implica

$$
\begin{aligned}
\int_{s_{i-1}}^{\vartheta} C(s) & \operatorname{Var}_{\theta \in[0, h]} \mu(s, \theta)\left\|y_{s}-x\left(\tau_{i}\right)_{s}\right\|_{\infty} d s \\
& = \begin{cases}0, & \vartheta \in\left[s_{i-1}, \tau_{i}\right], \\
\int_{\tau_{i}}^{\vartheta} C(s) \operatorname{Var}_{\theta \in[0, h]} \mu(s, \theta)\left\|y_{s}-x\left(\tau_{i}\right)_{s}\right\|_{\infty} d s, & \vartheta \in\left[\tau_{i}, s_{i}\right] .\end{cases}
\end{aligned}
$$

Também, dado um intervalo marcado particular $\left(\tau_{i},\left[s_{i-1}, s_{i}\right]\right)$, existem duas possibilidades:

(i) A interseção de $\left[s_{i-1}, s_{i}\right]$ e $\left\{t_{1}, \ldots, t_{m}\right\}$ contém só um ponto $t_{k}=\tau_{i}$.

(ii) A interseção de $\left[s_{i-1}, s_{i}\right]$ e $\left\{t_{1}, \ldots, t_{m}\right\}$ é vazio.

Agora, considere o caso (i): Como foi explicado antes, segue da definição do calibre $\delta$ que

$$
\begin{aligned}
& \int_{\tau_{i}}^{s_{i}} L(s)\left\|y_{s}-x\left(\tau_{i}\right)_{s}\right\|_{\infty} d g(s) \leqslant \frac{\epsilon}{4 m+1} \\
& \int_{\tau_{i}}^{s_{i}} C(s) \operatorname{Var}_{\theta \in[0, h]} \mu(s, \theta)\left\|y_{s}-x\left(\tau_{i}\right)_{s}\right\|_{\infty} d s<\frac{\epsilon}{4 m+1} .
\end{aligned}
$$

No caso (ii), observemos que

$$
\left\|y_{s}-x\left(\tau_{i}\right)_{s}\right\|_{\infty}=\sup _{\rho \in\left[\tau_{i}, s\right]}\left\|y(\rho)-y\left(\tau_{i}\right)\right\| \leqslant \epsilon, \quad s \in\left[\tau_{i}, s_{i}\right]
$$

pela definição do calibre $\delta$. Combinando os casos (i) e (ii) e usando o fato 
que o caso (i) acontece no máximo $2 m$ vezes, obtemos

$$
\begin{aligned}
& \left\|x(v)-x\left(t_{0}\right)-\sum_{i=1}^{l}\left(G\left(x\left(\tau_{i}\right), s_{i}\right)-G\left(x\left(\tau_{i}\right), s_{i-1}\right)\right)\right\|_{\infty} \\
& <\epsilon \int_{t_{0}}^{t_{0}+\sigma} L(s) d g(s)+\epsilon \int_{t_{0}}^{t_{0}+\sigma} C(s) \operatorname{Var}_{\theta \in[0, h]} \mu(s, \theta) d s+\frac{4 m \epsilon}{4 m+1} \\
& <\epsilon\left(\int_{t_{0}}^{t_{0}+\sigma} L(s) d g(s)+\int_{t_{0}}^{t_{0}+\sigma} C(s) \operatorname{Var}_{\theta \in[0, h]} \mu(s, \theta) d s+1\right)
\end{aligned}
$$

Como $\epsilon$ foi tomado arbitrariamente, segue que

$$
x(v)-x\left(t_{0}\right)=\int_{t_{0}}^{v} D G(x(\tau), t)
$$

e obtemos o resultado desejado.

Teorema 6.9. Considere $B_{c}$ e $P_{c}$ como em (H1) e suponha (H2). Sejam $\phi \in P_{c}$ e $G: B_{c} \times\left[t_{0}, t_{0}+\sigma\right] \rightarrow R\left(\left[t_{0}-h, t_{0}+\sigma\right], \mathbb{R}^{n}\right)$ dado por (6.8) e suponha que $G(x, t) \in R\left(\left[t_{0}-h, t_{0}+\sigma\right], \mathbb{R}^{n}\right)$ para cada $x \in B_{c}, t \in\left[t_{0}, t_{0}+\sigma\right]$. Seja $x:\left[t_{0}, t_{0}+\sigma\right] \rightarrow B_{c}$ uma solução da EDO generalizada

$$
\frac{d x}{d \tau}=D G(x, t)
$$

com condição inicial

$$
x\left(t_{0}\right)(\vartheta)= \begin{cases}\phi\left(\vartheta-t_{0}\right), & t_{0}-h \leqslant \vartheta \leqslant t_{0}, \\ x\left(t_{0}\right)\left(t_{0}\right), & t_{0} \leqslant \vartheta \leqslant t_{0}+\sigma .\end{cases}
$$

Então, a função $y \in B_{c}$ definido por

$$
y(\vartheta)= \begin{cases}x\left(t_{0}\right)(\vartheta), & t_{0}-h \leqslant \vartheta \leqslant t_{0}, \\ x(\vartheta)(\vartheta), & t_{0} \leqslant \vartheta \leqslant t_{0}+\sigma .\end{cases}
$$

é uma solução da EDFN em medida

$$
\left\{\begin{array}{l}
y(t)=y\left(t_{0}\right)+\int_{t_{0}}^{t} f\left(y_{s}, s\right) d g(s)+\int_{0}^{h} d_{\theta} \mu(t, \theta) y(t-\theta)-\int_{0}^{h} d_{\theta} \mu\left(t_{0}, \theta\right) y\left(t_{0}-\theta\right), \\
y_{t_{0}}=\phi
\end{array}\right.
$$

sobre $t \in\left[t_{0}-r, t_{0}+\sigma\right]$. 
Demonstração. A igualdade $y_{t_{0}}=\phi$ segue da definição de $y$ e $x\left(t_{0}\right)$. Resta provar que se $v \in\left[t_{0}, t_{0}+\sigma\right]$, então

$y(v)-y\left(t_{0}\right)=\int_{t_{0}}^{v} f\left(y_{s}, s\right) d g(s)+\int_{0}^{h} d_{\theta} \mu(v, \theta) y(v-\theta)-\int_{0}^{h} d_{\theta} \mu\left(t_{0}, \theta\right) y\left(t_{0}-\theta\right)$.

Usando o Lema 6.6, obtemos

$y(v)-y\left(t_{0}\right)=x(v)(v)-x\left(t_{0}\right)\left(t_{0}\right)=x(v)(v)-x\left(t_{0}\right)(v)=\left(\int_{t_{0}}^{v} D G(x(\tau), t)\right)(v)$.

Portanto,

$$
\begin{gathered}
y(v)-y\left(t_{0}\right)-\int_{t_{0}}^{v} f\left(y_{s}, s\right) d g(s)-\int_{0}^{h} d_{\theta} \mu(t, \theta) y(t-\theta)+\int_{0}^{h} d_{\theta} \mu\left(t_{0}, \theta\right) y\left(t_{0}-\theta\right) \\
=\left(\int_{t_{0}}^{v} D G(x(\tau), t)\right)(v)-\int_{t_{0}}^{v} f\left(y_{s}, s\right) d g(s) \\
\quad-\int_{0}^{h} d_{\theta} \mu(v, \theta) y(t-\theta)+\int_{0}^{h} d_{\theta} \mu\left(t_{0}, \theta\right) y\left(t_{0}-\theta\right)
\end{gathered}
$$

Seja $\epsilon>0$ arbitrário. Como $g$ é uma função não decrescente, que pode ter apenas um número finito de pontos $t \in\left[t_{0}, v\right]$ tais que $\Delta^{+} g(t) \geqslant \epsilon$; denotemos esses pontos por $t_{1}, \ldots, t_{m}$. Consideremos o calibre $\delta:\left[t_{0}, t_{0}+\sigma\right] \rightarrow \mathbb{R}^{+}$tal que

e

$$
\delta(\tau)<\min \left\{\frac{t_{k}-t_{k-1}}{2}, k=2, \ldots, m\right\}, \tau \in\left[t_{0}, t_{0}+\sigma\right]
$$

$$
\delta(\tau)<\min \left\{\left|\tau-t_{k}\right| ; k=1, \ldots, m\right\}, \quad \tau \in\left[t_{0}, t_{0}+\sigma\right] .
$$

Como na prova do teorema 6.8 , estas condições garantem que se o intervalo marcado $(\tau,[c, d])$ é $\delta$-fino, então $[c, d]$ contém, no máximo, um dos pontos $t_{1}, \ldots, t_{m}$, e, além disso $\tau=t_{k}$ quando $t_{k} \in[c, d]$.

De novo, o calibre $\delta$ pode ser escolhido de tal forma que

$$
\int_{t_{k}}^{t_{k}+\delta\left(t_{k}\right)} L(s)\left\|y_{s}-x\left(t_{k}\right)_{s}\right\|_{\infty} d g(s)<\frac{\epsilon}{2 m+1}, \quad k \in\{1, \ldots, m\} .
$$

De acordo com o Lema 6.5, a função $G$ dado por (6.8) pertence à classe $\mathcal{F}\left(\overline{B_{c}} \times\left[t_{0}, t_{0}+\sigma\right], h\right)$, onde

$$
h(t)=\int_{t_{0}}^{t}(L(s)+M(s)) d g(s)+\int_{t_{0}}^{t} C(s) \operatorname{Var}_{\theta \in[0, h]} \mu(s, \theta) d s\left(\|\tilde{x}\|_{\infty}+c\right) .
$$


Pela definição de $h$ dada por (6.14), segue imediatamente que para cada $\tau \in\left[t_{0}, t_{0}+\sigma\right] \backslash\left\{t_{1}, \ldots, t_{m}\right\}$, obtemos

$$
\|h(\tau+t)-h(\tau)\|<\epsilon
$$

Assim, podemos supor que o calibre $\delta$ satisfaz

$$
\|h(\rho)-h(\tau)\| \leqslant \epsilon, \quad \rho \in[\tau, \tau+\delta(\tau)) .
$$

Finalmente, o calibre $\delta$ deve ser tal que

$$
\left\|\int_{t_{0}}^{v} D G(x(\tau), t)-\sum_{i=1}^{l}\left(G\left(x\left(\tau_{i}\right), s_{i}\right)-G\left(x\left(\tau_{i}\right), s_{i-1}\right)\right)\right\|_{\infty}<\epsilon
$$

para cada partição $\delta$-fina $\left\{\left(\tau_{i},\left[s_{i-1}, s_{i}\right]\right), i=1, \ldots, l\right\}$ de $\left[t_{0}, v\right]$. A existência de tal calibre segue da definição da integral de Kurzweil-Henstock. Agora, escolhamos uma partição $\delta$-fina particular $\left\{\left(\tau_{i},\left[s_{i-1}, s_{i}\right]\right), i=1, \ldots, l\right\}$ de $\left[t_{0}, v\right]$. Pelas Equações (6.13) e (6.15), obtemos

$$
\begin{aligned}
& \mid y(v)-y\left(t_{0}\right)-\int_{t_{0}}^{v} f\left(y_{s}, s\right) d g(s) \\
& \quad-\int_{0}^{h} d_{\theta} \mu(v, \theta) y(v-\theta)+\int_{0}^{h} d_{\theta} \mu\left(t_{0}, \theta\right) y\left(t_{0}-\theta\right) \mid \\
& =\mid\left(\int_{t_{0}}^{v} D G(x(\tau), t)\right)(v)-\int_{t_{0}}^{v} f\left(y_{s}, s\right) d g(s) \\
& \quad-\int_{0}^{h} d_{\theta} \mu(v, \theta) y(v-\theta)+\int_{0}^{h} d_{\theta} \mu\left(t_{0}, \theta\right) y\left(t_{0}-\theta\right) \mid \\
& <\epsilon+\mid \sum_{i=1}^{l}\left(G\left(x\left(\tau_{i}\right), s_{i}\right)-G\left(x\left(\tau_{i}\right), s_{i-1}\right)\right)(v)-\int_{t_{0}}^{v} f\left(y_{s}, s\right) d g(s) \\
& \quad-\int_{0}^{h} d_{\theta} \mu\left(s_{i}, \theta\right) y\left(s_{i}-\theta\right)+\int_{0}^{h} d_{\theta} \mu\left(s_{i-1}, \theta\right)\left(y\left(s_{i-1}-\theta\right) \mid\right. \\
& \leqslant \epsilon+\sum_{i=1}^{l} \mid\left(G\left(x\left(\tau_{i}\right), s_{i}\right)-G\left(x\left(\tau_{i}\right), s_{i-1}\right)\right)(v)-\int_{s_{i-1}}^{s_{i}} f\left(y_{s}, s\right) d g(s) \\
& \quad-\int_{0}^{h} d_{\theta} \mu\left(s_{i}, \theta\right) y\left(s_{i}-\theta\right)+\int_{0}^{h} d_{\theta} \mu\left(s_{i-1}, \theta\right) y\left(s_{i-1}-\theta\right) \mid .
\end{aligned}
$$


A definição de $G$ fornece

$$
\begin{aligned}
\left(G\left(x\left(\tau_{i}\right), s_{i}\right)\right. & \left.-G\left(x\left(\tau_{i}\right), s_{i-1}\right)\right)(v)=\int_{s_{i-1}}^{s_{i}} f\left(x\left(\tau_{i}\right)_{s}, s\right) d g(s) \\
& -\int_{0}^{h} d_{\theta} \mu\left(s_{i}, \theta\right) x\left(\tau_{i}\right)\left(s_{i}-\theta\right)+\int_{0}^{h} d_{\theta} \mu\left(s_{i-1}, \theta\right) x\left(\tau_{i}\right)\left(s_{i-1}-\theta\right),
\end{aligned}
$$

o qual implica

$$
\begin{aligned}
& \mid\left(G\left(x\left(\tau_{i}\right), s_{i}\right)-G\left(x\left(\tau_{i}\right), s_{i-1}\right)\right)(v)-\int_{s_{i-1}}^{s_{i}} f\left(y_{s}, s\right) d g(s) \\
& \quad-\int_{0}^{h} d_{\theta} \mu\left(s_{i}, \theta\right) y\left(s_{i}-\theta\right)+\int_{0}^{h} d_{\theta} \mu\left(s_{i-1}, \theta\right) y\left(s_{i-1}-\theta\right) \mid \\
& \leqslant\left|\int_{s_{i-1}}^{s_{i}} f\left(x\left(\tau_{i}\right)_{s}, s\right) d g(s)-\int_{s_{i-1}}^{s_{i}} f\left(y_{s}, s\right) d g(s)\right| \\
& +\mid \int_{0}^{h} d_{\theta} \mu\left(s_{i}, \theta\right)\left[x\left(\tau_{i}\right)\left(s_{i}-\theta\right)-y\left(s_{i}-\theta\right)\right] \\
& \quad-\int_{0}^{h} d_{\theta} \mu\left(s_{i-1}, \theta\right)\left[x\left(\tau_{i}\right)\left(s_{i-1}-\theta\right)-y\left(s_{i-1}-\theta\right)\right] \mid .
\end{aligned}
$$

Pelo Lema 6.6, para cada $i \in\{1, \ldots, l\}, x\left(\tau_{i}\right)_{s}=x(s)_{s}=y_{s}$ para $s \in\left[s_{i-1}, \tau_{i}\right]$ e $y_{s}=x(s)_{s}=x\left(s_{i}\right)_{s}$ para $s \in\left[\tau_{i}, s_{i}\right]$. Portanto

$$
\begin{aligned}
\left|\int_{s_{i-1}}^{s_{i}}\left[f\left(x\left(\tau_{i}\right)_{s}, s\right)-f\left(y_{s}, s\right)\right] d g(s)\right| & =\left|\int_{\tau_{i}}^{s_{i}}\left[f\left(x\left(\tau_{i}\right)_{s}, s\right)-f\left(y_{s}, s\right)\right] d g(s)\right| \\
& =\left|\int_{\tau_{i}}^{s_{i}}\left[f\left(x\left(\tau_{i}\right)_{s}, s\right)-f\left(x\left(s_{i}\right)_{s}, s\right)\right] d g(s)\right| \\
& \leqslant \int_{\tau_{i}}^{s_{i}} L(s)\left\|x\left(\tau_{i}\right)_{s}-x\left(s_{i}\right)_{s}\right\|_{\infty} d g(s),
\end{aligned}
$$

onde a última desigualdade segue da condição (C). E também, obtemos

$$
\begin{aligned}
& \mid \int_{0}^{h} d_{\theta} \mu\left(s_{i}, \theta\right)\left[x\left(\tau_{i}\right)\left(s_{i}-\theta\right)-y\left(s_{i}-\theta\right)\right] \\
& \quad-\int_{0}^{h} d_{\theta} \mu\left(s_{i-1}, \theta\right)\left[x\left(\tau_{i}\right)\left(s_{i-1}-\theta\right)-y\left(s_{i-1}-\theta\right)\right] \mid
\end{aligned}
$$




$$
\begin{aligned}
& \leqslant \int_{s_{i-1}}^{s_{i}} C(s) \int_{0}^{h} d_{\theta} \mu(s, \theta)\left|x\left(\tau_{i}\right)(s-\theta)-y(s-\theta)\right| d s \\
& \leqslant \int_{s_{i-1}}^{s_{i}} C(s) \operatorname{Var}_{\theta \in[0, h]} \mu(s, \theta)\left\|x\left(\tau_{i}\right)_{s}-y_{s}\right\|_{\infty} d s \\
& =\int_{\tau_{i}}^{s_{i}} C(s) \operatorname{Var}_{\theta \in[0, h]} \mu(s, \theta)\left\|x\left(\tau_{i}\right)_{s}-y_{s}\right\|_{\infty} d s .
\end{aligned}
$$

Uma vez mais, distinguimos dois casos:

(i) A interseção de $\left[s_{i-1}, s_{i}\right]$ e $\left\{t_{1}, \ldots, t_{m}\right\}$ contém somente um ponto $t_{k}=$ $\tau_{i}$.

(ii) A interseção de $\left[s_{i-1}, s_{i}\right]$ e $\left\{t_{1}, \ldots, t_{m}\right\}$ é vazio.

No caso (i), segue da definição do calibre $\delta$ que

$$
\int_{\tau_{i}}^{s_{i}} L(s)\left\|y_{s}-x\left(\tau_{i}\right)_{s}\right\|_{\infty} d g(s) \leqslant \frac{\epsilon}{4 m+1} .
$$

Além disso, obtemos

$$
\int_{\tau_{i}}^{s_{i}} C(s) \operatorname{Var}_{\theta \in[0, h]} \mu(s, \theta)\left\|x\left(\tau_{i}\right)_{s}-y_{s}\right\|_{\infty} d s \leqslant \frac{\epsilon}{4 m+1} .
$$

Estas condições implicam

$$
\begin{aligned}
& \mid\left(G\left(x\left(\tau_{i}\right), s_{i}\right)-G\left(x\left(\tau_{i}\right), s_{i-1}\right)\right)(v)-\int_{s_{i-1}}^{s_{i}} f\left(y_{s}, s\right) d g(s) \\
& \quad-\int_{0}^{h} d_{\theta} \mu\left(s_{i}, \theta\right) y\left(s_{i}-\theta\right)+\int_{0}^{h} d_{\theta} \mu\left(s_{i-1}, \theta\right) y\left(s_{i-1}-\theta\right) \mid \leqslant \frac{\epsilon}{2 m+1} .
\end{aligned}
$$

No caso (ii), usamos o Lema 3.23 e a Observação 3.24 para obter a seguinte estimativa

$$
\left\|x\left(s_{i}\right)_{s}-x\left(\tau_{i}\right)_{s}\right\|_{\infty} \leqslant\left\|x\left(s_{i}\right)-x\left(\tau_{i}\right)\right\|_{\infty} \leqslant h\left(s_{i}\right)-h\left(\tau_{i}\right) \leqslant \epsilon
$$

para cada $s \in\left[\tau_{i}, s_{i}\right]$, e portanto

$$
\begin{gathered}
\mid\left(G\left(x\left(\tau_{i}\right), s_{i}\right)-G\left(x\left(\tau_{i}\right), s_{i-1}\right)\right)(v)-\int_{s_{i-1}}^{s_{i}} f\left(y_{s}, s\right) d g(s) \\
-\int_{0}^{h} d \mu\left(s_{i}, \theta\right) y\left(s_{i}-\theta\right)+\int_{0}^{h} d_{\theta} \mu\left(s_{i-1}, \theta\right) y\left(s_{i-1}-\theta\right) \mid \\
\leqslant \epsilon \int_{\tau_{i}}^{s_{i}} L(s) d g(s)+\epsilon \int_{\tau_{i}}^{s_{i}} C(s) \operatorname{Var}_{\theta \in[0, h]} \mu(s, \theta) d s .
\end{gathered}
$$


Combinando os casos (i), (ii) e usando o fato que o caso (i) acontece no máximo $2 m$ vezes, obtemos

$$
\begin{aligned}
\sum_{i=1}^{l} \mid(G( & \left.\left.x\left(\tau_{i}\right), s_{i}\right)-G\left(x\left(\tau_{i}\right), s_{i-1}\right)\right)(v)-\int_{s_{i-1}}^{s_{i}} f\left(y_{s}, s\right) d g(s) \\
& -\int_{0}^{h} d_{\theta} \mu\left(s_{i}, \theta\right) y\left(s_{i}-\theta\right)+\int_{0}^{h} d_{\theta} \mu\left(s_{i-1}, \theta\right) y\left(s_{i-1}-\theta\right) \mid \\
\leqslant & \epsilon \int_{t_{0}}^{t_{0}+\sigma} L(s) d g(s)+\epsilon \int_{t_{0}}^{t_{0}+\sigma} C(s) \operatorname{Var}_{\theta \in[0, h]} \mu(s, \theta) d s+\frac{2 m \epsilon}{2 m+1} .
\end{aligned}
$$

Consequentemente

$$
\begin{aligned}
\| y(v)-y\left(t_{0}\right) & -\int_{t_{0}}^{v} f\left(y_{s}, s\right) d g(s)-\int_{0}^{h} d_{\theta} \mu(v, \theta) y(v-\theta)+\int_{0}^{h} d_{\theta} \mu\left(t_{0}, \theta\right) y\left(t_{0}-\theta\right) \| \\
& <\epsilon\left(2+\int_{t_{0}}^{t_{0}+\sigma} L(s) d g(s)+\int_{t_{0}}^{t_{0}+\sigma} C(s) \operatorname{Var}_{\theta \in[0, h]} \mu(s, \theta) d s\right),
\end{aligned}
$$

o que completa a prova.

\subsection{Existência e unicidade de soluções}

Nesta seção, obteremos resultados de existência e unicidade de soluções para EDFNs em medida, usando a correspondência entre estas equações e as EDOs generalizadas, apresentada na seção anterior.

Começaremos lembrando o resultado sobre existência e unicidade para EDOs generalizadas, que será importante para a prova do próximo teorema.

Teorema 6.10 (Vide [28, Teo. 2.15]). Suponha que $X$ é um espaço de Banach, $O \subset X$ um conjunto aberto, e $G: O \times\left[t_{0}, t_{0}+\sigma\right] \rightarrow X$ pertencente a classe $\mathcal{F}\left(O \times\left[t_{0}, t_{0}+\sigma\right], h\right)$, onde $h:\left[t_{0}, t_{0}+\sigma\right] \rightarrow \mathbb{R}$ é uma função não decrescente e contínua a esquerda. Se $x_{0} \in O$ é tal que $x_{0}+G\left(x_{0}, t_{0}+\right)-G\left(x_{0}, t_{0}\right) \in O$, então existe $\delta>0$ e uma função $x:\left[t_{0}, t_{0}+\delta\right] \rightarrow X$ que é a única solução da EDO generalizada

$$
\frac{d x}{d \tau}=D G(x, t), \quad x\left(t_{0}\right)=x_{0}
$$


A seguir, mostraremos o teorema de existência e unicidade para equações diferenciais neutras em medida.

Teorema 6.11. Considere $B_{c}$ e $P_{c}$ como em (H1) e suponha (H2). Se $\phi \in P_{c}$ é tal que a função

$$
z(t)= \begin{cases}\phi\left(t-t_{0}\right), & t \in\left[t_{0}-h, t_{0}\right], \\ \phi(0)+f\left(\phi, t_{0}\right) \Delta^{+} g\left(t_{0}\right), & t \in\left(t_{0}, t_{0}+\sigma\right]\end{cases}
$$

pertence a $B_{c}$, então existe $\delta>0$ e uma função $y:\left[t_{0}-r, t_{0}+\delta\right] \rightarrow \mathbb{R}^{n}$ que é solução única da EDFN em medida

$$
\left\{\begin{array}{l}
y(t)=y\left(t_{0}\right)+\int_{t_{0}}^{t} f\left(y_{s}, s\right) d g(s)+\int_{0}^{h} d_{\theta} \mu(t, \theta) y(t-\theta)-\int_{0}^{h} d_{\theta} \mu\left(t_{0}, \theta\right) y\left(t_{0}-\theta\right), \\
y_{t_{0}}=\phi
\end{array}\right.
$$

Demonstração. Seja $G$ uma função definida por (6.8). Então, de acordo com o Lema 6.5, esta função pertence à classe $\mathcal{F}\left(B_{c} \times\left[t_{0}, t_{0}+\sigma\right], h\right)$, onde

$$
h(t)=\int_{t_{0}}^{t}(M(s)+L(s)) d g(s)+\int_{t_{0}}^{t} C(s) \operatorname{Var}_{\theta \in[0, h]} \mu(s, \theta) d s\|x\|_{\infty} .
$$

Além disso, seja

$$
x_{0}(\vartheta)= \begin{cases}\phi\left(\vartheta-t_{0}\right), & \vartheta \in\left[t_{0}-h, t_{0}\right], \\ \phi(0), & \vartheta \in\left[t_{0}, t_{0}+\sigma\right] .\end{cases}
$$

É evidente que $x_{0} \in B_{c}$. Também afirmamos que $x_{0}+G\left(x_{0}, t_{0}+\right)-G\left(x_{0}, t_{0}\right) \in$ $B_{c}$. Primeiro, notemos que $G\left(x_{0}, t_{0}\right)=0$. O limite $G\left(x_{0}, t_{0}+\right)$ é tomado com respeito à norma do supremo e sabemos que o limite existe desde que $G$ seja regrada em relação à segunda variável (isso decorre do fato de que $\left.G \in \mathcal{F}\left(B_{c} \times\left[t_{0}, t_{0}+\sigma\right], h\right)\right)$ e também, notemos que $J\left(x_{0}, t_{0}+\right)=0$, usando a condição $\left(\mathrm{A}^{*}\right)$. Assim, é suficiente calcular o limite pontual $F\left(x_{0}, t_{0}+\right)(\vartheta)$ para cada $\vartheta \in\left[t_{0}-h, t_{0}+\sigma\right]$. Usando o Teorema 6.7, obtemos

$$
F\left(x_{0}, t_{0}+\right)(\vartheta)= \begin{cases}0, & t \in\left[t_{0}-h, t_{0}\right], \\ f\left(\phi, t_{0}\right) \Delta^{+} g\left(t_{0}\right), & t \in\left(t_{0}, t_{0}+\sigma\right] .\end{cases}
$$


Segue que $x_{0}+G\left(x_{0}, t_{0}+\right)-G\left(x_{0}, t_{0}\right)=x_{0}+F\left(x_{0}, t_{0}+\right)=z \in B_{c}$.

Como todas as hipóteses do Teorema 6.10 estão satisfeitas, existe $\delta>0$ e uma única solução $x:\left[t_{0}, t_{0}+\delta\right] \rightarrow X$ da EDO generalizada

$$
\frac{d x}{d \tau}=D G(x, t), \quad x\left(t_{0}\right)=x_{0}
$$

De acordo com o Teorema 6.9, a função $y:\left[t_{0}-h, t_{0}+\delta\right] \rightarrow \mathbb{R}^{n}$ dada por

$$
y(\vartheta)= \begin{cases}x\left(t_{0}\right)(\vartheta), & t_{0}-h \leqslant \vartheta \leqslant t_{0}, \\ x(\vartheta)(\vartheta), & t_{0} \leqslant \vartheta \leqslant t_{0}+\delta\end{cases}
$$

é uma solução da equação diferencial funcional neutra em medida (6.16).

Esta solução deve ser única; de outro modo, o Teorema 6.8 implicaria que $x$ não é a única solução da EDO generalizada (6.17). Portanto, o resultado segue.

\subsection{Dependência contínua em relação aos parâmetros}

Nesta seção, utilizaremos o teorema existente de dependência contínua para EDOs generalizadas, para derivar as mesmas propriedades qualitativas para as EDFNs em medida.

Para isso, precisamos do seguinte teorema que pode ser encontrado em [39, Teorema 2.18].

Teorema 6.12. As seguintes condições são equivalentes:

1. Um conjunto $\mathcal{A} \subset R\left([\alpha, \beta], \mathbb{R}^{n}\right)$ é relativamente compacto.

2. $O$ conjunto $\{x(\alpha) ; x \in \mathcal{A}\}$ é limitado e existe uma função decrescente $\eta:[0, \infty) \rightarrow[0, \infty), \eta(0)=0$ e uma função crescente $K:[\alpha, \beta] \rightarrow \mathbb{R}$ tais que

$$
\left\|x\left(t_{2}\right)-x\left(t_{1}\right)\right\| \leqslant \eta\left(K\left(t_{2}\right)-K\left(t_{1}\right)\right)
$$

para cada $x \in \mathcal{A}, \alpha \leqslant t_{1} \leqslant t_{2} \leqslant \beta$.

O próximo resultado de dependência contínua para EDOs generalizadas é uma versão de [38, Teorema 2.4], em espaços de Banach. 
Teorema 6.13. Seja $X$ um espaço de Banach, $O \subset X$ um conjunto aberto, e $h_{k}:[a, b] \rightarrow \mathbb{R}, k=0,1,2, \ldots$ uma sequência de funções não decrescentes contínuas à esquerda tais que $h_{k}(b)-h_{k}(a) \leqslant c$ para algum $c>0$ e $k=$ $0,1,2, \ldots$ Suponha que $G_{k}: O \times[a, b] \rightarrow X$ pertence à classe $\mathcal{F}\left(O \times[a, b], h_{k}\right)$, $k=0,1,2 \ldots e$

$$
\begin{aligned}
\lim _{k \rightarrow \infty} G_{k}(x, t) & =G_{0}(x, t), \quad x \in O, t \in[a, b], \\
\lim _{k \rightarrow \infty} G_{k}(x, t+) & =G_{0}(x, t+) \quad x \in O, t \in[a, b),
\end{aligned}
$$

Para $k=1,2, \ldots$ seja $x_{k}:[a, b] \rightarrow O$ uma solução da EDO generalizada

$$
\frac{d x}{d \tau}=D G_{k}(x, t) \text {. }
$$

Se existe uma função $x_{0}:[a, b] \rightarrow O$ tal que $\lim _{k \rightarrow \infty} x_{k}(t)=x_{0}(t)$ uniformemente para $t \in[a, b]$, então $x_{0}$ é uma solução de

$$
\frac{d x}{d \tau}=D G_{0}(x, t), \quad t \in[a, b]
$$

Devemos observar que em [38, Teorema 2.4], as funções $G_{k}$ são definidas em $O \times(-T, T)$, onde $[a, b] \subset(-T, T)$, e similarmente as funções $h_{k}$ estão definidas no intervalo aberto $(-T, T)$. Contudo, é fácil estender as funções definidas sobre $[a, b]$ para $(-T, T)$ deixando $G_{k}(x, t)=G_{k}(x, a)$ para $t \in(-T, a)$, $G_{k}(x, t)=G_{k}(x, b)$ para $t \in(b, T)$, e analogamente para $h_{k}$. Notemos que as funções estendidas $G_{k}$ agora pertencem à classe $\mathcal{F}\left(O \times(-T, T), h_{k}\right)$, como em [38].

Agora estamos preparados para provar o teorema de dependência contínua para equações diferenciais funcionais neutras em medida.

Teorema 6.14. Considere $B_{c}$ e $P_{c}$ como em (H1) e sejam $g:\left[t_{0}, t_{0}+\sigma\right] \rightarrow \mathbb{R}$ uma função não decrescente contínua a esquerda, $f_{k}: P_{c} \times\left[t_{0}, t_{0}+\sigma\right] \rightarrow \mathbb{R}^{n}$, $k=0,1,2, \ldots$ uma sequência de funções que satisfazem as condições $(A)$, $(B)$ e $(C)$ para as funções $L, M:\left[t_{0}, t_{0}+\sigma\right] \rightarrow \mathbb{R}$ e $\mu_{k}: \mathbb{R} \times \mathbb{R} \rightarrow \mathbb{R}^{n \times n} a$ função normalizada que satisfaz a condição $\left(A^{*}\right)$, para a função $C:\left[t_{0}, t_{0}+\right.$ $\sigma] \rightarrow \mathbb{R}$ para $k=0,1,2 \ldots$ Além disso, suponha que para cada $y \in B_{c}$, os limites

$$
\begin{aligned}
& \lim _{k \rightarrow \infty} \int_{t_{0}}^{t} f_{k}\left(y_{s}, s\right) d g(s)=\int_{t_{0}}^{t} f_{0}\left(y_{s}, s\right) d g(s) \\
& \lim _{k \rightarrow \infty} \int_{0}^{h} d_{\theta} \mu_{k}(t, \theta) y(t-\theta)=\int_{0}^{h} d_{\theta} \mu_{0}(t, \theta) y(t-\theta)
\end{aligned}
$$


uniformemente com respeito a $t \in\left[t_{0}, t_{0}+\sigma\right]$.

Para $k=0,1,2 \ldots$ consideremos a sequência de funções $\phi_{k} \in P_{c}$, tais que $\lim _{k \rightarrow \infty} \phi_{k}=\phi_{0}$ uniformemente em $[-h, 0]$. Seja $y_{k} \in B_{c}$, uma solução de

$$
\left\{\begin{aligned}
y_{k}(t)= & y_{k}\left(t_{0}\right)+\int_{t_{0}}^{t} f_{k}\left(\left(y_{k}\right)_{s}, s\right) d g(s)+\int_{0}^{h} d_{\theta}\left[\mu_{k}(t, \theta)\right] y_{k}(t-\theta) \\
& -\int_{0}^{h} d_{\theta}\left[\mu_{k}\left(t_{0}, \theta\right)\right] y_{k}\left(t_{0}-\theta\right) \\
\left(y_{k}\right)_{t_{0}}= & \phi_{k}
\end{aligned}\right.
$$

em $\left[t_{0}-r, t_{0}+\sigma\right]$. Se existe uma função $y_{0} \in B_{c}$ tal que $\lim _{k \rightarrow \infty} y_{k}=y_{0} \mathrm{em}$ $\left[t_{0}, t_{0}+\sigma\right]$, então $y_{0}:\left[t_{0}-r, t_{0}+\sigma\right] \rightarrow \mathbb{R}^{n}$ é uma solução de

$$
\left\{\begin{aligned}
y_{0}(t)= & y_{0}\left(t_{0}\right)+\int_{t_{0}}^{t} f_{0}\left(\left(y_{0}\right)_{s}, s\right) d g(s)+\int_{0}^{h} d_{\theta}\left[\mu_{0}(t, \theta)\right] y_{0}(t-\theta) \\
& -\int_{0}^{h} d_{\theta}\left[\mu_{0}\left(t_{0}, \theta\right)\right] y_{0}\left(t_{0}-\theta\right) \\
\left(y_{0}\right)_{t_{0}}= & \phi_{0} .
\end{aligned}\right.
$$

Demonstração. As hipóteses implicam que para cada $x \in B_{c}, \lim _{k \rightarrow \infty} G_{k}(x, t)=$ $G_{0}(x, t)$ uniformemente com respeito a $t \in\left[t_{0}, t_{0}+\sigma\right]$. Pelo teorema de Moore-Osgood (vide [85, Seç. 3.11]), $\lim _{k \rightarrow \infty} G_{k}(x, t+)=G_{0}(x, t+)$ para cada $x \in B_{c}$ e $t \in\left[t_{0}, t_{0}+\sigma\right)$. E também, $G_{0}(x, t) \in R\left(\left[t_{0}-r, t_{0}+\sigma\right], \mathbb{R}^{n}\right)$.

Segundo o Lema $6.5, G_{k} \in \mathcal{F}\left(B_{c} \times\left[t_{0}, t_{0}+\sigma\right], h\right)$ para cada $k=1,2, \ldots$ onde

$$
h(t)=\int_{t_{0}}^{t}(M(s)+L(s)) d g(s)+\int_{t_{0}}^{t} C(s) \operatorname{Var}_{\theta \in[0, h]} \mu(s, \theta) d s\|x\|_{\infty},
$$

para $t \in\left[t_{0}, t_{0}+\sigma\right]$. Como $\lim _{k \rightarrow \infty} G_{k}(x, t)=G_{0}(x, t)$, então $G_{0} \in \mathcal{F}(O \times$ $\left.\left[t_{0}, t_{0}+\sigma\right], h\right)$.

Para cada $k=0,1,2, \ldots, t \in\left[t_{0}, t_{0}+\sigma\right]$, seja

$$
x_{k}(t)(\vartheta)=\left\{\begin{array}{l}
y_{k}(\vartheta), \vartheta \in\left[t_{0}-r, t\right] \\
y_{k}(t), \vartheta \in\left[t, t_{0}+\sigma\right] .
\end{array}\right.
$$

Conforme o Teorema 6.8, $x_{k}$ é uma solução da EDO generalizada

$$
\frac{d x}{d \tau}=D G_{k}(x, t)
$$


Para $k=1,2, \ldots$ e $t_{0} \leqslant t_{1} \leqslant t_{2} \leqslant t_{0}+\sigma$, obtemos

$$
\begin{gathered}
\left\|y_{k}\left(t_{2}\right)-y_{k}\left(t_{1}\right)\right\|=\| \int_{t_{1}}^{t_{2}} f_{k}\left(\left(y_{k}\right)_{s}, s\right) d g(s)+\int_{0}^{h} d_{\theta} \mu_{k}\left(t_{2}, \theta\right) y_{k}\left(t_{2}-\theta\right) \\
\quad-\int_{0}^{h} d_{\theta} \mu_{k}\left(t_{1}, \theta\right) y_{k}\left(t_{1}-\theta\right) \| \\
\leqslant h\left(t_{2}\right)-h\left(t_{1}\right)<h\left(t_{2}\right)-h\left(t_{1}\right)+\left(t_{2}-t_{1}\right),
\end{gathered}
$$

onde $\eta(t)=t$ para $t \in[0, \infty)$ e $K(t)=h(t)+t, t \in\left[t_{0}, t_{0}+\sigma\right]$. Portanto, segue que $K$ é uma função crescente. Além disso, a sequência $\left\{y_{k}\left(t_{0}\right)\right\}_{k=1}^{\infty}$ é limitada. Assim, a condição 2 do Teorema 6.12 é satisfeita e segue que $\left\{y_{k}\right\}_{k=1}^{\infty}$ contem uma subsequência que é uniformemente convergente em $\left[t_{0}, t_{0}+\sigma\right]$. Sem perda de generalidade, podemos denotar esta subsequência de novo por $\left\{y_{k}\right\}_{k=1}^{\infty}$. Como $\left(y_{k}\right)_{t_{0}}=\phi_{k}$, segue que $\left\{y_{k}\right\}_{k=1}^{\infty}$ de fato é uniformemente convergente em $\left[t_{0}-h, t_{0}+\sigma\right]$.

Pela definição de $x_{k}$, obtemos

$$
\lim _{k \rightarrow \infty} x_{k}(t)=x_{0}(t)
$$

uniformemente com respeito a $t \in\left[t_{0}, t_{0}+\sigma\right]$. Segue do Teorema 6.13 que $x_{0}$ é uma solução de

$$
\frac{d x}{d \tau}=D G_{0}(x, t)
$$

sobre o intervalo $\left[t_{0}, t_{0}+\sigma\right]$. A prova termina aplicando o Teorema 6.9, que garante que $y_{0}$ satisfaz (6.18), o que conclui a prova.

\subsection{Um exemplo}

Por meio de um exemplo ilustraremos a correspondência entre a equação diferencial funcional neutra em medida e sua correspondente equação diferencial ordinária generalizada, tal como foi determinado na Seção 6.2, calculando explicitamente a solução da EDO generalizada.

Consideremos a EDFN em medida

$$
\begin{aligned}
D[y(t)-a y(t-1)] & =b y(t-1) D u, \quad t \geqslant 0 \\
y_{0} & =\varphi
\end{aligned}
$$

onde

$$
u(s)=s+H_{1}(s)
$$


com $H_{1}$ como sendo a função de Heaviside dada por $H_{1}(s)=0$ se $s \leqslant 1$ e $H_{1}(s)=1$ caso contrário. A solução de (6.19) satisfaz a equação integral

$$
y(t)=y(0)+a y(t-1)-a y(-1)+\int_{0}^{t} b y(s-1) d u(s)
$$

Fixemos $T>0$. Escreveremos a EDFN em medida (6.19) como a EDO generalizada

$$
\begin{aligned}
\frac{d x}{d \tau} & =D G(x, t), \\
x(0)(\vartheta) & =\varphi([-1, \vartheta, 0]),
\end{aligned}
$$

onde para cada $t \in[0, T], x(t)$ é uma função definida no intervalo $[-1, T]$. Além disso, $G(x, t)=F(x, t)+J(x, t) \operatorname{com} F$ e $J$ definidas em (6.9) e (6.10) respectivamente, que no caso particular da EDFN em medida (6.19) são dados por

$$
\begin{aligned}
F(x, t)(\vartheta) & =\int_{0}^{[0, \vartheta, t]} b x(s-1) d u(s), \\
J(x, t)(\vartheta) & =a x([0, \vartheta, t]-1)-a x(-1) .
\end{aligned}
$$

A forma integral de (6.21) é

$$
x(t)=x(0)+\int_{0}^{t} D G(x(\tau), s)=x(0)+\int_{0}^{t} D F(x(\tau), s)+\int_{0}^{t} D J(x(\tau), s) .
$$

Observemos que para todo $t \in[0, T]$

$$
x(t)(\vartheta)=\varphi(\vartheta), \quad \vartheta \in[-1,0]
$$

como neste caso $[0, \vartheta, t]=0$ e segue de $(6.22)$ e (6.23) que

$$
\left(\int_{0}^{t} D G(x(\tau), s)\right)(\vartheta)=0
$$

Consideremos agora o caso $0<t \leqslant 1$. Notemos que $u$ restrita ao intervalo $[0,1]$ é a função identidade, de forma que $d u(s)=d s$ para $s \in[0,1]$.

Para calcular $\int_{0}^{t} D F(x(\tau), s)$ e $\int_{0}^{t} D G(x(\tau), s)$, consideramos a partição $0=s_{0}<s_{1}<\cdots<s_{n}=b$ do intervalo $[0, t]$. Para uma escolha arbitrária 
de marcas $\tau_{i} \in\left[s_{i-1}, s_{i}\right]$, calculamos a soma de Kurzweil para $F$

$$
\begin{aligned}
\sum_{i=1}^{n}\left[F\left(x\left(\tau_{i}\right), s_{i}\right)(\vartheta)-F\left(x\left(\tau_{i}\right), s_{i-1}\right)(\vartheta)\right] & =\sum_{i=1}^{n} \int_{\left[0, \vartheta, s_{i-1}\right]}^{\left[0, \vartheta, s_{i}\right]} b x\left(\tau_{i}\right)(s-1) d s \\
& =\sum_{i=1}^{n} \int_{s_{i-1}}^{\left[s_{i-1}, \vartheta, s_{i}\right]} b x\left(\tau_{i}\right)(s-1) d s \\
& =\int_{0}^{[0, \vartheta, t]} b \varphi(s-1) d s
\end{aligned}
$$

onde a última identidade é obtida usando (6.24) e considerando os diferentes casos $\vartheta<0, \vartheta>t$ e $\vartheta \in\left[s_{k-1}, s_{k}\right]$ para algum inteiro $0<k \leqslant n$. Em outras palavras, a soma de Kurzweil é independente da partição particular e portanto

$$
\int_{0}^{t} D F(x(\tau), s)=\int_{0}^{[0, \vartheta, t]} b \varphi(s-1) d s .
$$

Analogamente, a soma de Kurzweil para $J$ em alguma partição é

$$
\begin{aligned}
\sum_{i=1}^{n}\left[J\left(x\left(\tau_{i}\right), s_{i}\right)(\vartheta)\right. & \left.-J\left(x\left(\tau_{i}\right), s_{i-1}\right)(\vartheta)\right] \\
& =a \sum_{i=1}^{n}\left[x\left(\tau_{i}\right)\left(\left[0, \vartheta, s_{i}\right]-1\right)-x\left(\tau_{i}\right)\left(\left[0, \vartheta, s_{i-1}\right]-1\right)\right] \\
& =a \sum_{i=1}^{n}\left[\varphi\left(\left[0, \vartheta, s_{i}\right]-1\right)-\varphi\left(\left[0, \vartheta, s_{i-1}\right]-1\right)\right] \\
& =\varphi([0, \vartheta, t]-1)-a \varphi(-1)
\end{aligned}
$$

assim,

$$
\int_{0}^{t} D J(x(\tau), s)=a \varphi([0, \vartheta, t]-1)-a \varphi(-1) .
$$

Portanto,

$$
\begin{aligned}
x(t)(\vartheta) & =x(0)(\vartheta)+\int_{0}^{t} D F(x(\tau), s)+\int_{0}^{t} D J(x(\tau), s) \\
& =\varphi([-1, \vartheta, 0])+\int_{0}^{[0, \vartheta, t]} b \varphi(s-1) d s+\varphi([0, \vartheta, t]-1)-a \varphi(-1) \\
& =\varphi([-1, \vartheta, t]), \quad 0 \leqslant t \leqslant 1,
\end{aligned}
$$


onde usamos de novo que $\varphi$ é solução da EDFN

$$
\frac{d}{d t}[y(t)-a y(t-1)]=b y(t-1)
$$

sujeita à condição inicial $y_{0}=\varphi$, cuja forma integral lê-se como

$$
\varphi(t)-a \varphi(t-1)-\varphi(0)+a \varphi(-1)=\int_{0}^{t} b \varphi(s-1) d s, \quad t>0,
$$

e pode ser resolvida pela método dos passos.

Agora, consideremos o caso $1<t \leqslant 2$. Observemos que

$$
\begin{aligned}
F\left(x\left(\tau_{i}\right), s_{j}\right)(\vartheta) & =\int_{0}^{\left[0, \vartheta, s_{j}\right]} b x\left(\tau_{i}\right)(s-1) d s+\int_{0}^{\left[0, \vartheta, s_{j}\right]} b x\left(\tau_{i}\right)(s-1) d H_{1}(s) \\
& =\int_{0}^{\left[0, \vartheta, s_{j}\right]} b x\left(\tau_{i}\right)(s-1) d s+b \varphi(0) H_{1}\left(s_{j}\right) .
\end{aligned}
$$

Calculando a soma de Kurzweil como antes, porém tomando em conta o último termo em (6.28), obtemos

$$
\int_{1}^{t} D F(x(\tau), s)=\int_{1}^{[1, \vartheta, t]} b \varphi(s-1) d s+b \varphi(0) .
$$

Usando os mesmos cálculos como no caso $0<t \leqslant 1$ obtemos

$$
\begin{aligned}
\int_{1}^{t} D J(x(\tau), s) & =\int_{0}^{t} D J(x(\tau), s)-\int_{0}^{1} D J(x(\tau), s) \\
& =a \varphi([0, \vartheta, t]-1)-a \varphi([0, \vartheta, 1]-1) \\
& =a \varphi([1, \vartheta, t]-1)-a \varphi(0) .
\end{aligned}
$$

De (6.29) e (6.30), lembrando que $\varphi$ é solução da EDFN (6.26), conseguimos para $t \in(1,2]$,

$$
\begin{aligned}
x(t)(\vartheta) & =\varphi([-1, \vartheta, 1])+\int_{1}^{[1, \vartheta, t]} b \varphi(s-1) d s+a \varphi([1, \vartheta, t]-1)-a \varphi(0)+b \varphi(0) \\
& =\varphi([-1, \vartheta, t])+b \varphi(0), \quad 1<t \leqslant 2 .
\end{aligned}
$$

Finalmente, consideramos o caso $t>2$. Destacamos que $x(2)(\vartheta)=\varphi(\vartheta)+$ $b \varphi(0)$ para $\vartheta \in(1,2]$. A linearidade da $F$ e $J$ em $x$ nos permite dividir 
o problema. Para $t \in[0,2]$, colocando $x_{1}(t)(\vartheta)=\varphi([-1, \vartheta, t])$ e $x_{2}(t)=$ $a \varphi(0) H_{1}([0, \vartheta, t])$, tais que $x(t)=x_{1}(t)+x_{2}(t)$ para $t \in[0,2]$. Observamos que para $s>2$, de (6.20) conseguimos que $d u(s)=d s$, portanto não acontecem saltos na solução para frente.

Usando os mesmos cálculos como anteriormente, para $t>2$ obtemos

$$
\begin{aligned}
x_{1}(t)(\vartheta) & =x_{1}(2)(\vartheta)+\left(\int_{2}^{t} D G\left(x_{1}(\tau), s\right)\right)(\vartheta) \\
& =\varphi([-1, \vartheta, 2])+\int_{2}^{[2, \vartheta, t]} b \varphi(s-1) d s+a \varphi([2, \vartheta, t]-1)-a \varphi(1) \\
& =\varphi([-1, \vartheta, 2])+\varphi([2, \vartheta, t])-\varphi(2) \\
& =\varphi([-1, \vartheta, t]), \quad t>2 .
\end{aligned}
$$

Definindo a função $w(s)=a \varphi(0) H_{1}(s)$ para $-1 \leqslant s \leqslant 2$, e chamando de novo por $w$ a solução de (6.26) sujeita à condição inicial $y_{2}=w_{2}$, obtemos $x_{2}(t)(\theta)=w([-1, \vartheta, t])$ for $t \in[0,2]$. Colocando $w$ no lugar de $y$ nos cálculos prévios temos

$$
\begin{aligned}
x_{2}(t)(\vartheta) & =x_{2}(2)(\vartheta)+\left(\int_{2}^{t} D G\left(x_{2}(\tau), s\right)\right)(\vartheta) \\
& =w([-1, \vartheta, t]), \quad t>2 .
\end{aligned}
$$

Consequentemente, para $t>0$, obtemos

$$
x(t)(\vartheta)=\varphi([-1, \vartheta, t])+w([-1, \vartheta, t]) .
$$

Uma substituição da função $y(t)=\varphi(t)+w(t)$ na forma integral (6.27) mostra que é a solução da EDFN em medida (6.19). 


\section{Apêndice A \\ Introdução à teoria de semigrupos fortemente contínuos e seus adjuntos}

\section{A.1 Definições}

Nesta seção, apresentaremos a teoria básica de semigrupos de operadores lineares limitados fortemente contínuos.

Seja $X$ um espaço de Banach complexo e seja, para cada $t \geqslant 0, T(t)$ : $X \rightarrow X$ um operado linear limitado.

Definição A.1. Uma família $\{T(t)\}_{t \geqslant 0}$ é chamada um semigrupo fortemente contínuo, ou $\mathcal{C}_{0}$-semigrupo sempre que as seguintes propriedades estejam satisfeitas

1. $T(0)=I$,

2. $T(t) T(s)=T(t+s)$, para $t, s \geqslant 0$,

3. para todo $x \in X,\|T(t) x-x\| \rightarrow 0$, quando $t \downarrow 0$.

Definição A.2. O gerador infinitesimal $A$ de $\{T(t)\}_{t \geqslant 0}$ é definido por

$$
\begin{aligned}
\mathcal{D}(A) & =\left\{x \mid \lim _{h \downarrow 0} \frac{1}{h}(T(h) x-x)\right\}, \\
A x & =\lim _{h \downarrow 0} \frac{1}{h}(T(h) x-x)
\end{aligned}
$$


Portanto $A$ é a derivada de $T(t)$ em $t=0$, e em geral, é ilimitado. A seguir, enumeraremos as propriedades básicas do semigrupo $\{T(t)\}_{t \geqslant 0}$, do seu gerador infinitesimal $A$ e as suas inter-relações.

Proposição A.3. Se $T(t)$ é um $\mathcal{C}_{0}$-semigrupo. Então

1. (Vide [66, Teorema 2.2]) Existem constantes $\omega \geqslant 0$ e $M \geqslant 1$ tal que

$$
\|T(t)\| \leqslant M e^{\omega t}
$$

2. (Vide [66, Corolário 2.3]) Para cada $x \in X, t \mapsto T(t) x$ é uma função contínua de $\mathbb{R}_{+}$(reta real não negativa) em $X$.

A princípio, $\mathcal{D}(A)$ poderia conter somente $x=0$. Como primeiro passo para provar que $\mathcal{D}(A)$ é suficientemente grande, observemos.

Proposição A.4 (Vide [66, Teorema 2.4]). Sejam $t>0$ arbitrário, $T(t)$ um $\mathcal{C}_{0}$-semigrupo e A seu gerador infinitesimal. Então

1. Para $x \in X$,

$$
\lim _{h \downarrow 0} \frac{1}{h} \int_{t}^{t+h} T(s) x d s=T(t) x .
$$

2. Para qualquer $x \in X, \int_{0}^{t} T(s) x d s \in \mathcal{D}(A) e$

$$
A\left(\int_{0}^{t} T(s) x d s\right)=T(t) x-x .
$$

3. $\mathcal{D}(A)$ é $T(t)$-invariante e para $x \in \mathcal{D}(A)$

$$
\frac{d}{d t} T(t) x=A T(t) x=T(t) A x .
$$

4. Para $x \in \mathcal{D}(A)$,

$$
T(t) x-T(s) x=\int_{s}^{t} T(\tau) A x d \tau=\int_{s}^{t} A T(\tau) x d \tau .
$$

Corolário A.5 (Vide [66, Corolário 2.5]). Se A é gerador infinitesimal de um $\mathcal{C}_{0}$-semigrupo $T(t)$. Então $\mathcal{D}(A)$ é denso em $X$ e $A$ é um operado linear fechado. 


\section{A.2 Espaço dual e Semigrupos adjuntos}

O espaço dos funcionais lineares contínuos em $X$ é chamado espaço dual de $X$ e denotado por $X^{*}$. Um elemento do espaço $X^{*}$ será denotado por $x^{*}$. Muniremos o espaço $X^{*}$ com a norma

$$
\left\|x^{*}\right\|=\sup _{\|x\| \leqslant 1}\left|\left\langle x^{*}, x\right\rangle\right|
$$

$\operatorname{assim}\left(X^{*},\|\cdot\|\right)$ é um espaço de Banach. Pelo Teorema de Hanh-Banach,

$$
\|x\|=\sup _{\|x\| \leqslant 1}\left|\left\langle x^{*}, x\right\rangle\right|
$$

A partir da norma sobre $X^{*}$, devemos trabalhar com a chamada topologia fraca-*.

Se $A: X \rightarrow X$ é um operador ilimitado, densamente definido então o operador adjunto $A^{*}$ é definido por:

$$
D\left(A^{*}\right)=\left\{x^{*} \in X^{*}, \exists y^{*} \in X^{*}\right\}
$$

e então $y^{*}=A^{*} x^{*}$.

Proposição A.6 (Vide [7, Proposição 1.4.4.a]).

1. $A^{*}$ é fraco-* fechado, isto é, o gráfico de $A^{*}$ é fechado em $X^{*} \times X^{*}$ munido com a topologia fraca-*.

2. Se A é fechado. Então $\mathcal{D}\left(A^{*}\right)$ é fraco-* denso.

Voltando aos semigrupos. Sejam $T(t)$ um $\mathcal{C}_{0^{-}}$semigrupo em $X$ e $T^{*}(t):=$ $(T(t))^{*}$ os operador adjuntos sobre o espaço dual $X^{*}$.

\section{Proposição A.7.}

1. (Vide [7, Proposição 1.4.3]) A familia de operadores adjuntos $T^{*}(t)$ é um semigrupo fraca-* contínuo. Ou seja, $T^{*}(0)=I, T^{*}(t) T^{*}(s)=$ $T^{*}(t+s)$, para $t, s \geqslant 0$ e para $x^{*} \in X^{*}, t \mapsto T^{*}(t) x^{*}$ é contínua de $\mathbb{R}_{+}$ em $X^{*}$ munido com a topologia fraca-*. 
2. (Vide [7, Proposição 1.4.4.c]) Seja A o gerador infinitesimal do semigrupo $T(t)$. Então o dual de $A, A^{*}$, é o gerador fraco-* do semigrupo adjunto $T^{*}$, isto é,

$$
\frac{1}{t}\left\langle T^{*}(t) x^{*}-x^{*}, x\right\rangle
$$

converge para todo $x \in X$, quando $t \downarrow 0$ se e somente se $x^{*} \in \mathcal{D}\left(A^{*}\right)$. Neste caso, $\lim _{t \downarrow 0} \frac{1}{t}\left\langle T^{*}(t) x^{*}-x^{*}, x\right\rangle=\left\langle A^{*} x^{*}, x\right\rangle$.

3. (Vide [7, Proposição 1.4.4.b]) $\mathcal{D}\left(A^{*}\right)$ é $T^{*}(t)$ invariante e $A^{*} T^{*}(t)=$ $T^{*}(t) A^{*}$ em $\mathcal{D}\left(A^{*}\right)$. Além disso,

$$
\left\langle T^{*}(t) x^{*}-x^{*}, x\right\rangle=\int_{0}^{t}\left\langle T^{*}(\sigma) A^{*} x^{*}, x\right\rangle d \sigma .
$$

Definição A.8. Seja $S$ um operador linear em $X$ e seja $Y$ um subespaço de $X$. O operador $\tilde{S}$ definido por $\mathcal{D}(\tilde{S})=\{x \in \mathcal{D}(S) \cap Y \mid S x \in Y\}$ e $\tilde{S} x=S x$, para $x \in \mathcal{D}(\tilde{S})$ é chamado parte de $S$ em $Y$.

O operador $A^{*}$ não é necessariamente densamente definido. Contudo, apresentaremos agora o teorema que garante que a parte de $A^{*}$ em $\overline{\mathcal{D}\left(A^{*}\right)}$ gera um $\mathcal{C}_{0}$-semigrupo.

Definição A.9. $X^{\odot}=\left\{x^{*} \in X^{*} \mid \lim _{t \downarrow 0}\left\|T^{*}(t) x^{*}-x^{*}\right\|=0\right\}$

Proposição A.10 (Vide [7, Proposição 1.4.7]).

1. $X^{\odot} \subset X^{*}$, fechado na norma e $T^{*}(t)$ invariante.

2. $X^{\odot}=\overline{\mathcal{D}\left(A^{*}\right)}$.

3. $A^{\odot}$ é a parte de $A^{*}$ em $X^{\odot}$ em $X^{\odot}$, isto é,

$$
\mathcal{D}\left(A^{\odot}\right)=\left\{x^{*} \in \mathcal{C}\left(A^{*}\right) \mid A^{*} x^{*} \in X^{\odot}\right\}, \quad A^{\odot} x^{*}=A^{*} x^{*} .
$$

Observação A.11. Será denotado por $T^{\odot}(t)$ o $\mathcal{C}_{0}$-semigrupo em $X^{\odot}$ obtido pela restrição de $T^{*}(t)$ ao subespaço fechado invariante $X^{\odot}$.

A partir do $\mathcal{C}_{0}$-semigrupo $T^{\odot}(t)$ definido em $X^{\odot}$, podemos repetir a construção descrita anteriormente e introduzir o semigrupo $T^{\odot *}(t)$ em $X^{\odot}$ e $T^{\odot \odot}$ em $X^{\odot \odot}$. Assim, podemos definir a aplicação linear contínua $j: X \rightarrow X^{\odot *}$ por

$$
\left\langle j x, x^{\odot}\right\rangle=\left\langle x^{\odot}, x\right\rangle .
$$

Como $X^{\odot}$ é fraco-* denso em $X^{*}$, a aplicação $j$ é injetiva. 


\section{A.3 A integral fraca-*}

Consideremos $X$ um espaço de Banach, e $X^{*}$ seu adjunto. Sejam $a, b \in \mathbb{R}$ com $b>a$ e $q:[a, b] \rightarrow X^{*}$ tal que

$$
\langle q(\cdot), x\rangle \in L^{1}([a, b], \mathbb{C}), \quad \forall x \in X .
$$

Mostraremos que existe $Q \in X^{*}$ tal que

$$
\langle Q, x\rangle=\int_{a}^{b}\langle q(\sigma), x\rangle d \sigma, \quad \forall x \in X
$$

e chamaremos $Q$ a integral fraca-* de $q$ sobre o intervalo $[a, b]$. Com efeito, consideremos a aplicação linear

$$
\begin{aligned}
& \mathcal{L}: X \rightarrow L^{1}([a, b], \mathbb{C}) \\
& x \mapsto \mathcal{L} x \quad:[a, b] \\
& t \quad \rightarrow \mathbb{C} \\
& t \quad \mapsto(\mathcal{L} x)(t)=\langle q(t), x\rangle
\end{aligned}
$$

Agora, notemos que $\mathcal{L}$ é uma aplicação linear fechada. De fato, sejam $x_{n} \rightarrow$ $x \in X$, e $\mathcal{L} x_{n} \rightarrow g \in L^{1}([a, b], \mathbb{C})$. Como $q(t)$ é limitada e $x_{n} \rightarrow x$, então $\left\langle q(t), x_{n}\right\rangle \rightarrow\langle q(t), x\rangle$, ou seja $\left(\mathcal{L} x_{n}\right)(t) \rightarrow(\mathcal{L} x)(t)$ em $\mathbb{C}$. Segue da unicidade que $g(t)=(\mathcal{L} x)(t)$, para quase todo $t \in[a, b]$.

Assim, pelo Teorema do Gráfico Fechado, concluímos que $\mathcal{L}$ é limitado, isto é

$$
\|\mathcal{L} x\|_{L^{1}}=\int_{a}^{b}|(\mathcal{L} x)(\sigma)| d \sigma=\int_{a}^{b}|\langle q(\sigma), x\rangle| d \sigma<K\|x\|_{X}
$$

para alguma constante positiva $K$.

Consequentemente, a aplicação $x \mapsto \int_{a}^{b}\langle q(\sigma), x\rangle d \sigma$, define um funcional linear contínuo, e $Q \in X^{*}$ debe existir, tal que

$$
\langle Q, x\rangle=\int_{a}^{b}\langle q(\sigma), x\rangle d \sigma
$$

Para simplificar denotaremos a integral fraca-* como segue

$$
Q=\int_{a}^{b} q(\sigma) d \sigma
$$

Uma desvantagem da integral fraca-* é a não comutatividade com operadores lineares limitados em $X^{*}$. Entretanto, temos o seguinte resultado, 
Lema A.12 (Vide [24, Ap. II, Lema 3.14]). Seja $L: X \rightarrow X$ um operador linear limitado. Então

$$
L^{*} \int_{a}^{b} q(\sigma) d \sigma=\int_{a}^{b} L^{*} q(\sigma) d \sigma
$$

Demonstração. Seja $x \in X$, então

$$
\begin{aligned}
\left\langle L^{*} \int_{a}^{b} q(\sigma) d \sigma, x\right\rangle & =\left\langle\int_{a}^{b} q(\sigma) d \sigma, L x\right\rangle=\int_{a}^{b}\langle q(\sigma), L x\rangle d \sigma \\
& =\int_{a}^{b}\left\langle L^{*} q(\sigma), x\right\rangle d \sigma=\left\langle\int_{a}^{b} L^{*} q(\sigma) d \sigma, x\right\rangle
\end{aligned}
$$

o que completa a demonstração do lema.

Na sequência, apresentaremos algumas propriedades da integral fraca-*. Seja $K$ uma família de operadores lineares limitados fortemente contínua em $\mathrm{X}$, isto é, para todo $x \in X$, a função $\sigma \mapsto K(\sigma) x$ é contínua de $[a, b]$ em $X$. Seja $h:[a, b] \rightarrow X^{*}$ contínua na norma. Interpretaremos a integral

$$
\int_{a}^{b} K(\sigma)^{*} h(\sigma) d(\sigma)
$$

como uma integral fraca-*. Para isso, observemos que

$$
\left\langle\int_{a}^{b} K(\sigma)^{*} h(\sigma) d \sigma, x\right\rangle=\int_{a}^{b}\left\langle K(\sigma)^{*} h(\sigma), x\right\rangle d \sigma=\int_{a}^{b}\langle h(\sigma), K(\sigma) x\rangle d \sigma .
$$

Da hipótese sobre $K$, segue que existe uma constante positiva $M$ tal que $\|K(\sigma) x\| \leqslant M$ e sendo $h$ contínua na norma segue a limitação de $\langle h(\sigma), K(\sigma) x\rangle$ no intervalo compacto $[a, b]$. Portanto, $\left\langle K(\sigma)^{*} h(\sigma), x\right\rangle \in L^{1}([a, b], \mathbb{C})$ e $($ A.4 $)$ é sua integral fraca-*.

Observação A.13. Cabe mencionar que, sob as hipóteses acima, podemos trabalhar com a integral de Riemann desde que o integrando seja uma função contínua.

Agora, consideremos $f: \mathbb{R}_{+} \rightarrow X^{\odot *}$ uma função contínua na norma. Mostraremos que para $s, r \in \mathbb{R}$ tal que $0 \leqslant r \leqslant s \leqslant t<\infty$

$$
\int_{r}^{s} T_{0}^{\odot *}(t-\tau) f(\tau) d \tau
$$


esta bem definida como uma integral fraca-*. Com efeito, dado $x^{\odot} \in X^{\odot}$, observemos que

$$
\begin{aligned}
\left\langle\int_{r}^{s} T_{0}^{\odot *}(t-\tau) f(\tau) d \tau, x^{\odot}\right\rangle & =\int_{r}^{s}\left\langle T_{0}^{\odot *}(t-\tau) f(\tau), x^{\odot}\right\rangle d \tau \\
& =\int_{r}^{s}\left\langle f(\tau), T_{0}^{\odot}(t-\tau) x^{\odot}\right\rangle d \tau
\end{aligned}
$$

Sabemos que $T^{\odot}(t)$ é um $\mathcal{C}_{0}$-semigrupo e sendo $f$ contínua na norma temos que $\left\langle T_{0}^{\odot *}(t-\tau) f(\tau), x^{\odot}\right\rangle \in L^{1}$ e (A.5) é sua integral fraca-*.

Lema A.14. Sejam $x^{*} \in X^{*}, g \in L^{1}([a, b], \mathbb{C})$ e $K$ uma família de operadores lineares limitados fortemente contínua em $X$, então $\int_{a}^{b} K(\sigma)^{*} g(\sigma) x^{*} d \sigma \in X^{*}$ define uma integral fraca-*. Além disso,

$$
\left\langle\int_{a}^{b} K(\sigma)^{*} g(\sigma) x^{*} d \sigma, x\right\rangle=\int_{a}^{b} g(\sigma)\left\langle x^{*}, K(\sigma) x\right\rangle d \sigma, \quad \forall x \in X
$$

Demonstração. Inicialmente, notemos que

$$
\begin{aligned}
K(\sigma)^{*}: X^{*} & \rightarrow X^{*} \\
x^{*} \mapsto K(\sigma)^{*} x^{*}: & X \rightarrow \mathbb{C} \\
x & \mapsto\left\langle K(\sigma)^{*} x^{*}, x\right\rangle .
\end{aligned}
$$

Portanto, das hipóteses sobre $g$ e $K$, segue que $\left\langle K(\sigma)^{*} g(\sigma) x^{*}, x\right\rangle \in L^{1}$, consequentemente a integral fraca-* $\int_{a}^{b} K(\sigma)^{*} g(\sigma) x^{*} d \sigma \in X^{*}$ esta bem definida e

$$
\begin{aligned}
\left\langle\int_{a}^{b} K(\sigma)^{*} g(\sigma) x^{*} d \sigma, x\right\rangle & =\int_{a}^{b}\left\langle K(\sigma)^{*} g(\sigma) x^{*}, x\right\rangle d \sigma \\
& =\int_{a}^{b} g(\sigma)\left\langle x^{*}, K(\sigma) x\right\rangle d \sigma
\end{aligned}
$$

o que completa a demonstração do lema.

Lema A.15. Sejam $\eta \in L^{1}, x^{\odot *} \in X^{\odot *}$ e $T^{\odot *}$ o semigrupo dual do $\mathcal{C}_{0}$ semigrupo $T^{\odot}$. Para $r, s \in \mathbb{R}_{+}$, tal que $r \leqslant s \leqslant t<\infty$ temos que a integral

$$
\int_{r}^{s} T_{0}^{\odot *}(t-\tau) \eta(\tau) x^{\odot *} d \tau
$$

define uma integral fraca-* com valores em $X^{\odot *}$. 
Demonstração. Seja $x^{\odot} \in X^{\odot}$, das hipóteses sobre $\eta$ e levando em conta que $T^{\odot}$ é limitado, temos que $\left\langle T_{0}^{\odot *}(t-\tau) \eta(\tau) x^{\odot *}, x^{\odot}\right\rangle \in L^{1}$. Portanto a integral fraca-* de $T_{0}^{\odot *}(t-\tau) \eta(\tau) x^{\odot *}$ esta bem definida e

$$
\begin{aligned}
\left\langle\int_{r}^{s} T_{0}^{\odot *}(t-\tau) \eta(\tau) x^{\odot *} d \tau, x^{\odot}\right\rangle & =\int_{r}^{s}\left\langle T_{0}^{\odot * *}(t-\tau) \eta(\tau) x^{\odot *}, x^{\odot}\right\rangle d \tau \\
& =\int_{r}^{s} \eta(\tau)\left\langle x^{\odot *}, T_{0}^{\odot}(t-\tau) x^{\odot}\right\rangle d \tau,
\end{aligned}
$$

o que completa a prova.

Usando o Lema A.12 observemos que para a integral fraca-* dado pela Equação (A.4), temos

$$
L^{*} \int_{a}^{b} K(\sigma)^{*} h(\sigma) d \sigma=\int_{a}^{b} L^{*} K(\sigma)^{*} h(\sigma) d \sigma
$$

onde $L: X \rightarrow X$ é um operador linear limitado. Vale a pena lembrar que não é permitido substituir $L^{*}$ por qualquer operador linear limitado em $X^{*}$. Para finalizar, enunciaremos o seguinte lema, cuja prova segue diretamente da observação acima e da definição de semigrupo.

Lema A.16. Seja $f: \mathbb{R}_{+} \rightarrow X^{\odot *}$ contínua na norma. Então

$$
T_{0}^{\odot *}(h) \int_{r}^{s} T_{0}^{\odot *}(t-\tau) f(\tau) d \tau=\int_{r}^{s} T_{0}^{\odot *}(t+h-\tau) f(\tau) d \tau .
$$

\section{A.4 Variantes da fórmula da variação das constan- tes}

Considerando

$$
T(t) \varphi=T_{0}(t) \varphi+\int_{0}^{t} T_{0}^{\odot *}(t-\tau) B T(\tau) \varphi d \tau .
$$

Nesta seção, reuniremos identidades que serão derivadas do processo de inverter $\left\{T_{0}(t)\right\}$ e $\{T(t)\}$ na equação (A.6). Portanto, é de esperar-se que a identidade

$$
T(t) \varphi=T_{0}(t) \varphi+\int_{0}^{t} T^{\odot *}(t-\tau) B T_{0}(\tau) \varphi d \tau .
$$


ainda seja satisfeita. Tomando adjuntos em (A.6), e fazendo mudança de variável obtemos

$$
\begin{aligned}
T^{\odot}(t) x^{\odot} & =T_{0}^{\odot}(t) x^{\odot}+\int_{0}^{t} T^{*}(\tau) B^{*} T_{0}^{\odot}(t-\tau) \varphi d \tau \\
& =T_{0}^{\odot}(t) x^{\odot}+\int_{0}^{t} T^{*}(t-\tau) B^{*} T_{0}^{\odot}(\tau) \varphi d \tau .
\end{aligned}
$$

Por outro lado, (A.7) fornece se seguinte identidade

$$
T^{\odot}(t) x^{\odot}=T_{0}^{\odot}(t) x^{\odot}+\int_{0}^{t} T_{0}^{*}(t-\tau) B^{*} T^{\odot}(\tau) x^{\odot} d \tau .
$$

Assim, temos quatro variantes da fórmula da variação dos parâmetros, as quais carregam a mesma informação.

Em geral, não é possível derivar equações para os semigrupos fraco-* contínuos $T^{*}$ e $T^{\odot *}$, agindo nos espaços $X^{*}$ e $X^{\odot *}$, respetivamente. No entanto, para o semigrupo integrado $W(t): X^{\odot *} \rightarrow X$, definido por

$$
W(t) x^{\odot *}=\int_{0}^{t} T^{\odot *}(\tau) x^{\odot *} d \tau
$$

temos uma fórmula da variação das constantes que faz sentido no espaço maior $X^{\odot *}$

Teorema A.17 (Vide [24, Theorema III.2.16]). Para cada $x^{\odot *} \in X^{\odot *}$ e $t \geqslant 0$, temos

$$
W(t) x^{\odot *}=W_{0}(t) x^{\odot *} x^{\odot *}+\int_{0}^{t} T_{0}^{\odot *}(t-\tau) B W(\tau) x^{\odot *} d \tau .
$$

A prova deste teorema requer alguns lemas técnicos, convidamos ao leitor consultar a o livro de Diekmann et al [24, Seção III.2 ], para maiores detalhes.

Quando trabalhamos com EDFRs não autônomas, consideramos famílias de operadores a dois parâmetros $\{U(t, s)\}$ e também temos uma fórmula da variação das constantes dada por

$$
U(t, s) \varphi=T_{0}(t-s) \varphi+\int_{s}^{t} T_{0}^{\odot *}(t-\tau) B(\tau) U(\tau, s) \varphi d \tau
$$


Sejam

$$
\begin{aligned}
W(t, s) x^{\odot *} & =\int_{s}^{t} U^{\odot *}(\tau, s) x^{\odot *} d \tau, \\
W_{0}(t, s) x^{\odot *} & =\int_{s}^{t} T_{0}^{\odot *}(\tau-s) x^{\odot *} d \tau .
\end{aligned}
$$

Lema A.18. Suponha que $t \mapsto B(t)$ seja contínua de $[\alpha, \omega]$ em $\mathcal{L}\left(X, X^{\odot *}\right)$. Para todo $x^{*} \in X^{*}, x^{\odot *} \in X^{\odot *}, t \geqslant s$,

$$
\left\langle x^{*}, \int_{s}^{t} U^{\odot *}(\tau, s) d \tau\right\rangle=\left\langle x^{\odot *}, \int_{s}^{t} U^{*}(\tau, s) x^{*} d \tau\right\rangle .
$$

Demonstração. Fixemos $x^{*}=x^{\odot} \in X^{\odot}$. Da definição da integral fraca-* segue que

$$
\begin{aligned}
\left\langle x^{*}, \int_{s}^{t} U^{\odot *}(\tau, s) d \tau\right\rangle & =\int_{s}^{t}\left\langle x^{*}, U^{\odot *}(\tau, s) x^{\odot *}\right\rangle d \tau=\int_{s}^{t}\left\langle U^{\odot}(\tau, s) x^{*}, x^{\odot *}\right\rangle d \tau \\
& =\left\langle\int_{s}^{t} U^{\odot}(\tau, s) d \tau x^{*}, x^{\odot *}\right\rangle=\left\langle\int_{s}^{t} U^{*}(\tau, s) d \tau x^{*}, x^{\odot *}\right\rangle .
\end{aligned}
$$

Agora, notemos que para $x^{*} \in X^{\odot}, \frac{1}{h} \int_{0}^{h} U^{*}(s, \sigma) x^{*} d \sigma \in X^{\odot}$. Com efeito, da hipótese sob a família de operadores $B(t)$ segue de [24, Teorema 4.5] que $U^{*}(t, s)$ deixa invariante $X^{\odot}$, donde $U^{*}(s, \sigma) \in X^{\odot}$, portanto

$$
\begin{aligned}
\| U^{*}(t, s) & \frac{1}{h} \int_{0}^{h} U^{*}(s, \sigma) x^{*} d \sigma-\frac{1}{h} \int_{0}^{h} U^{*}(s, \sigma) x^{*} d \sigma \| \\
& =\frac{1}{h}\left\|\int_{0}^{h} U^{*}(t, s) U^{*}(s, \sigma) x^{*}-U^{*}(s, \sigma) x^{*} d \sigma\right\| \\
& \leqslant \frac{1}{h} \int_{0}^{h}\left\|U^{*}(t, s) U^{*}(s, \sigma) x^{*}-U^{*}(s, \sigma) x^{*}\right\| \rightarrow 0, \text { quando } t \rightarrow s .
\end{aligned}
$$

Portanto podemos aproximar $x^{*}$ por $\frac{1}{h} \int_{0}^{h} U^{*}(s, \sigma) x^{*} d \sigma \in X^{\odot}$ e no lado esquerdo de (A.13) temos

$$
\begin{aligned}
\left\langle\frac{1}{h} \int_{0}^{h} U^{*}(\sigma, s) x^{*} d \sigma, \int_{s}^{t} U^{\odot *}(\tau, s) x^{*} d \tau\right\rangle & =\frac{1}{h} \int_{0}^{h}\left\langle U^{*}(\sigma, s) x^{*}, \int_{s}^{t} U^{\odot *}(\tau, s) x^{*} d \tau\right\rangle \\
& =\frac{1}{h} \int_{0}^{h}\left\langle x^{*}, U(\sigma, s) \int_{s}^{t} U^{\odot *}(\tau, s) x^{*} d \tau\right\rangle .
\end{aligned}
$$


Como $\int_{s}^{t} U^{\odot *}(\tau, s) x^{*} d \tau \in X$ e sendo $U(\sigma, s)$ fortemente contínuo, temos

$$
\left\langle\frac{1}{h} \int_{0}^{h} U^{*}(\sigma, s) x^{*} d \sigma, \int_{s}^{t} U^{\odot *}(\tau, s) x^{*} d \tau\right\rangle \rightarrow\left\langle x^{*}, \int_{s}^{t} U^{\odot *}(\tau, s) x^{\odot *} d \tau\right\rangle, h \downarrow 0 .
$$

Da última igualdade do lado direito de A.13, para $x^{*}$ aproximado por $\frac{1}{h} \int_{0}^{h} U^{*}(s, \sigma) x^{*} d \sigma \in X^{\odot}$, obtemos

$$
\left\langle x^{\odot *}, \int_{s}^{t} U^{*}(\tau, s)\left(\frac{1}{h} \int_{0}^{h} U^{*}(s, \sigma) x^{*} d \sigma\right) d \tau\right\rangle \rightarrow\left\langle x^{\odot *}, \int_{s}^{t} U^{*}(\theta, s) x^{*} d \theta\right\rangle, h \downarrow 0 .
$$

Finalizando a prova do lema.

Corolário A.19. $W(t)^{*}$ aplica $X^{*}$ em $X^{\odot}$ e $W(t)^{*}=W^{\odot}(t)$, onde $W^{\odot}(t)$ : $X^{*} \rightarrow X^{\odot}$ é definido por

$$
W^{\odot}(t, s) x^{*}=\int_{s}^{t} U^{*}(\tau, s) x^{*} d \tau .
$$

Corolário A.20. Seja $\left\{x_{n}^{\odot *}\right\}$ uma sequência em $X^{\odot *}$ convergindo fraco-* para $x_{\infty}^{\odot *}$. Então $\left\{W(t) x_{n}^{\odot *}\right\}$ converge fracamente em $X$ para $W(t) x_{\infty}^{\odot *}$.

Demonstração. Para $x^{*} \in X^{\odot}$ e usando o corolário anterior, notemos que

$$
\begin{aligned}
\left\langle W(t, s) x_{n}^{\odot *}, x^{*}\right\rangle & =\left\langle\int_{s}^{t} U^{\odot *}(\tau, s) x_{n}^{\odot *} d \tau, x^{*}\right\rangle=\int_{s}^{t}\left\langle U^{\odot *}(\tau, s) x_{n}^{\odot *}, x^{*}\right\rangle d \tau \\
& =\int_{s}^{t}\left\langle x_{n}^{\odot *}, U^{\odot}(\tau, s) x^{*}\right\rangle d \tau=\left\langle x_{n}^{\odot *}, \int_{s}^{t} U^{\odot}(\tau, s) x^{*}\right\rangle d \tau \\
& \rightarrow\left\langle x_{\infty}^{\odot *}, \int_{s}^{t} U^{\odot}(\tau, s) x^{*} d \tau\right\rangle=\left\langle x_{\infty}^{\odot *}, \int_{s}^{t} U^{*}(\tau, s) x^{*} d \tau\right\rangle \\
& =\left\langle x_{\infty}^{\odot *}, W(t)^{*} x^{*}\right\rangle=\left\langle W(t) x_{\infty}^{\odot *}, x^{*}\right\rangle
\end{aligned}
$$

o que conclui a prova do corolário.

Agora provaremos uma fórmula da variação das constantes para o semigrupo $W(t, s)$.

Teorema A.21. Para cada $x^{\odot *} \in X^{\odot *}, t \geqslant s$,

$$
W(t, s) x^{\odot *}=W_{0}(t, s) x^{\odot *}+\int_{s}^{t} T_{0}^{\odot *}(t-\tau) B(\tau) W(\tau, s) x^{\odot *} d \tau .
$$


Demonstração. Da fórmula da variação das constantes, para $\varphi \in X$ e $x^{\odot} \in$ $X^{\odot}$

$$
\begin{aligned}
\left\langle x^{\odot}, \int_{s}^{t} U(\tau, s) \varphi d \tau\right\rangle= & \left\langle x^{\odot}, \int_{s}^{t} T_{0}(\tau-s) \varphi d \tau\right\rangle \\
& +\left\langle x^{\odot}, \int_{s}^{t} \int_{s}^{\tau} T_{0}^{\odot *}(\tau-\sigma) B(\sigma) U(\sigma, s) \varphi d \sigma d \tau\right\rangle \\
= & \left\langle x^{\odot}, \int_{s}^{t} T_{0}(\tau-s) \varphi d \tau\right\rangle \\
& +\int_{s}^{t} \int_{s}^{\tau}\left\langle B(\sigma)^{*} T_{0}^{\odot}(\tau-\sigma) x^{\odot}, U(\sigma, s) \varphi\right\rangle d \sigma d \tau .
\end{aligned}
$$

Mudando a ordem de integração no último termo, temos

$$
\left\langle x^{\odot}, W(t, s) \varphi\right\rangle=\left\langle x^{\odot}, W_{0}(t, s) \varphi\right\rangle+\int_{s}^{t}\left\langle B^{*}(t) T_{0}^{\odot}(\sigma) x^{\odot}, W(t-\sigma, s) \varphi\right\rangle d \sigma .
$$

Seja $x^{\odot *} \in X^{\odot *}$ e seja $\left\{x_{n}\right\}$ uma sequência em $X$ convergindo para $x^{\odot *}$ no sentido fraco-* (por exemplo tome $\left.x_{n}=n\left(n I-A^{\odot *}\right)^{-1} x^{\odot *}\right)$. Na última identidade considere $\varphi=x^{n}$. Segue do Corolário A.20 e do teorema da convergência dominada que

$\left\langle x^{\odot}, W(t, s) x^{\odot *}\right\rangle=\left\langle x^{\odot}, W_{0}(t, s) x^{\odot *}\right\rangle+\int_{s}^{t}\left\langle B^{*}(t) T_{0}^{\odot}(\sigma) x^{\odot}, W(t-\sigma, s) x^{\odot *}\right\rangle d \sigma$,

donde (A.14) segue imediatamente.

Corolário A.22. Para $x^{\odot *} \in X^{\odot *}$,

$$
\begin{aligned}
\int_{s}^{t} U^{\odot *}(t, \tau) x^{\odot *} d \tau= & \int_{s}^{t} T_{0}^{\odot *}(t-\tau) x^{\odot *} d \tau \\
& +\int_{s}^{t} T_{0}^{\odot * *}(t-\tau) B(\tau) \int_{s}^{\tau} U^{\odot *}(\tau, \sigma) x^{\odot *} d \sigma d \tau .
\end{aligned}
$$

Demonstração. Para $t=s$ a igualdade é satisfeita. Seja $x^{\odot} \in X^{\odot}$, integrando 
ambos lados de $s$ até $t$ e fazendo dualidade $\operatorname{com} x^{\odot}$, segue do Teorema A.21,

$$
\begin{aligned}
& \int_{s}^{t}\left\langle\int_{s}^{\sigma} U^{\odot *}(\sigma, \tau) x^{\odot *} d \tau-\int_{s}^{\sigma} T_{0}^{\odot *}(\sigma-\tau) x^{\odot *} d \tau, x^{\odot}\right\rangle d \sigma \\
& =\int_{s}^{t}\left\langle\left(W(t, \sigma)-W_{0}(t, \sigma)\right) x^{\odot *}, x^{\odot}\right\rangle d \sigma \\
& =\left\langle\int_{s}^{t}\left(W(t, \sigma)-W_{0}(t, \sigma)\right) x^{\odot *} d \sigma, x^{\odot}\right\rangle \\
& =\left\langle\int_{s}^{t} \int_{\sigma}^{t} T_{0}^{\odot *}(t-\tau) B(\tau) W(\tau, \sigma) x^{\odot *} d \tau d \sigma, x^{\odot}\right\rangle \\
& =\left\langle\int_{s}^{t} \int_{s}^{\gamma} T_{0}^{\odot *}(\gamma-\tau) B(\tau) \int_{s}^{\tau} U^{\odot *}(\tau, \sigma) x^{\odot *} d \sigma d \tau d \gamma, x^{\odot}\right\rangle
\end{aligned}
$$

donde segue o resultado. 


\section{Referências Bibliográficas}

[1] S. M. Afonso; E. M. Bonotto; M. Federson and L. P. Gimenes, Boundedness of solutions of retarded functional differential equations with variable impulses via generalized ordinary differential equations. Math. Nachr. 285 (2012), no. 5-6, 545-561.

[2] S. M. Afonso, E. M. Bonotto, M. Federson, S. Schwabik, Discontinuous local semiflows for Kurzweil equations leading to LaSalle's invariance principle for differential systems with impulses at variable times. J. Differential Equations 250 (2011), no. 7, 2969-3001.

[3] A. A. Andronov, A. G. Mă̈r, The simplest linear systems with retardation. Avtomatika i Telemehanika 7, (1946). 95-106.

[4] Z. Artstein, Topological dynamics of an ordinary differential equation and Kurzweil equations, J. Diff. Eq., 23 (1977), 224-243.

[5] R. Bellman, k. L. Cooke, Differential-difference equations. Academic Press, New York-London 1963

[6] N. N. Bogolyubov, A. Mitropolskii, Assymptotic Methods in the Theory of Non-linear Oscillations, Moscou, Nauka, 1963 (em russo).

[7] P. L. Butzer, H. Berens, Semi-groups of operators and approximation. Springer-Verlag, New York Inc., New York 1967

[8] A. Callender, A. G. Stevenson, Time lag in a control system. Proc. Soc. Chem. Ind. 18(1), 108-117, 1939.

[9] C. S. Hönig Volterra Stieltjes Integral Equations, North Holland Publ. Comp., Amsterdam, 1975. 
[10] Coppel, W. A. Almost periodic properties of ordinary differential equations. Ann. Mat. Pura Appl. (4) 761967 27-49.

[11] Coppel, W. A. Dichotomies in stability theory. Lecture Notes in Mathematics, Vol. 629. Springer-Verlag, Berlin-New York, 1978.

[12] Coppel, W. A. Dichotomies and reducibility. J. Differential Equations 3 1967 500-521.

[13] Coppel, W. A. Dichotomies and reducibility. II. J. Differential Equations 41968 386-398.

[14] Coppel, W. A. Stability and asymptotic behavior of differential equations. D. C. Heath and Co., Boston, Mass. 1965

[15] Coffman, Charles V.; Schäffer, Juan Jorge Dichotomies for linear difference equations. Math. Ann. 1721967 139-166.

[16] M. A. Cruz, J. K. Hale, Asymptotic behavior of neutral functional differential equations, Arch. Rational Mech. Anal., Vol. 34, (1969), 331-353.

[17] Ju. L. Daleckij, M. G. Kreŭn, Stability of solutions of differential equations in Banach space. Translated from the Russian by S. Smith. Translations of Mathematical Monographs, Vol. 43. American Mathematical Society, Providence, R.I., 1974.

[18] P. C. Das, R. R. Sharma, Existence and stability of measure differential equations, Czech. Math. Journal 22 (97) (1972), 145-158

[19] Clément, Ph.; Diekmann, O.; Gyllenberg, M.; Heijmans, H. J. A. M.; Thieme, H. R. Perturbation theory for dual semigroups. I. The sunreflexive case. Math. Ann. 277 (1987), no. 4, 709-725.

[20] Ph. Clément, O. Diekmann, M. Gyllenberg, H. J. A. M. Heijmans, H. R. Thieme, Perturbation theory for dual semigroups. II. Timedependent perturbations in the sun-reflexive case. Proc. Roy. Soc. Edinburgh Sect. A 109 (1988), no. 1-2, 145-172.

[21] Ph. Clément, O. Diekmann, M. Gyllenberg, H. J. A. M. Heijmans, H. R. Thieme, Perturbation theory for dual semigroups. III. Nonlinear Lipschitz continuous perturbations in the sun-reflexive [case]. Volterra integrodifferential equations in Banach spaces and applications (Trento, 
1987), 67-89, Pitman Res. Notes Math. Ser., 190, Longman Sci. Tech., Harlow, 1989.

[22] Ph. Clément, O. Diekmann, M. Gyllenberg, H. J. A. M. Heijmans, H. R. Thieme, Perturbation theory for dual semigroups. IV. The intertwining formula and the canonical pairing. Semigroup theory and applications (Trieste, 1987), 95-116, Lecture Notes in Pure and Appl. Math., 116, Dekker, New York, 1989.

[23] O. Diekmann, M. Gyllenberg, H. R. Thieme, Perturbation theory for dual semigroups. V. Variation of constants formulas. Semigroup theory and evolution equations (Delft, 1989), 107-123 Lecture Notes in Pure and Appl. Math., 135, Dekker, New York, 1991.

[24] O. Diekmann, S. A. van Gils, S. M. Verduyn Lunel, H.-O., Walther; Delay equations. Functional, complex, and nonlinear analysis. Applied Mathematical Sciences, 110. Springer-Verlag, New York, 1995.

[25] Diekmann, O. Perturbed dual semigroups and delay equations. Dynamics of infinite-dimensional systems (Lisbon, 1986), 67-73, NATO Adv. Sci. Inst. Ser. F Comput. Systems Sci., 37, Springer, Berlin, 1987.

[26] R. D. Driver, "Some harmless delays," in Delay and functional differential equations and their applications (Proc. Conf., Park City, Utah, 1972), pp. 103-119, New York: Academic Press, 1972.

[27] R. D. Driver, D. W. Sasser, M. L. Slater, "The equation $x^{\prime}(t)=a x(t)+$ $b x(t-\tau)$ with "small" delay," Amer. Math. Monthly, vol. 80, pp. 990-995, 1973 .

[28] M. Federson, Š. Schwabik, Generalized ODE approach to impulsive retarded functional differential equations, Differential and Integral Equations 19 (11), (2006), 1201-1234.

[29] M. Federson, Š. Schwabik, Stability for retarded functional differential equations, Ukrainian Math J., 244 (2008), 2334-2349.

[30] M. Federson, Š. Schwabik, A new approach to impulsive retarded differential equations: stability results. Functional Differential Equations, 16(4), (2009), 583-607. 
[31] M. Federson, M. Frasson, J. Mesquita, P. Tacuri, Measure neutral functional differential equations as generalized ODEs. Submetido para publicação.

[32] M. Federson, J. G. Mesquita, A. Slavik, Measure functional differential equations and functional dynamic equations on time scales, J. Diff. Equations, 252 (2012), 3816- 3847.

[33] M. Federson, J. G. Mesquita, E. Toon, Stability for measure retarded differential equations and for retarded dynamic equations on time scales. Submetido para publicação.

[34] M. Federson, J. G. Mesquita, E.Toon, Lyapunov stability for a class of measure retarded differential equations and for retarded dynamic equations on time scales. Pre-print.

[35] M. Federson, J. Mesquita, E. Toon, Stability results for measure functional differential equations via kurzweil-equations. Pre-print.

[36] M. Federson and P. Z. Táboas, Topological dynamics of retarded functional differential equations, J. Diff. Equations, 195(2), (2003), 313-331.

[37] M. Federson, E. Toon, Existence and uniqueness of a solution of functional differential equations with Kurzweil-Henstock integrable right-hand sides and variable impulses. Submetido para publicação.

[38] D. Fraňková, Continuous dependence on a parameter of solutions of generalized differential equations, Časopis pro pěstování matematiky, Vol. 114 (1989), No. 3, 230-261.

[39] D. Fraňková, Regulated functions, Mathematica Bohemica, No. 1, 20-59, 116 (1991).

[40] M. V. S. Frasson, Sistemas Impulsivos do Ponto de Vista das Equações Diferenciais em Medida, Dissertação de Mestrado, ICMC-USP, 2000.

[41] M. V. S. Frasson, On the dominance of roots of characteristic equations for neutral functional differential equations, Appl. Math. Comput., vol. 214, no. 1, pp. 66-72, 2009. 
[42] M. V. S. Frasson, S. M. Verduyn Lunel, Large time behaviour of linear functional differential equations, Integral Equations Operator Theory, vol. 47, no. 1, pp. 91-121, 2003.

[43] M. Frasson, P. H. Tacuri, Asymptotic behaviour of solutions to linear neutral delay differential equations with periodic coefficients. Submetido.

[44] L. P. Gimenes, Estabilidade e Oscilação de Soluções de Equações Diferenciais com Retardos e Impulsos, Tese de Doutorado, ICMC-USP, 2007.

[45] J. K. Hale and S. M. Verduyn Lunel, Introduction to functionaldifferential equations, vol. 99 of Applied Mathematical Sciences. New York: Springer-Verlag, 1993.

[46] J. K. Hale, A class of neutral equations with the fixed point property, Proc. Nat. Acad. Sci. U.S.A., Vol. 67, (1970), 136-137.

[47] J. K. Hale, K. R. Meyer, A class of functional equations of neutral type, Memoirs of the American Mathematical Society, No. 76, 1967.

[48] J. K. Hale, S. M. Verduyn Lunel, Averaging in infinite dimensions, J. of Integral Eq. and Appl., 2 (1990), 463-494.

[49] D. Henry, Linear autonomous neutral functional differential equations, J. Differential Equations., 15, (1974), 106-128.

[50] E. Hille, R. Phillips, Functional analysis and semi-groups. American Mathematical Society Colloquium Publications, vol. 31. American Mathematical Society, Providence, R. I., 1957.

[51] C. Imaz and Z. Vorel, Generalized ordinary differential equations in Banach spaces and applications to functional equations, Bol. Soc. Mat. Mexicana, 11 (1966), 47-59.

[52] V. Kolmanovskii, A. Myshkis, Introduction to the theory and applications of functional-differential equations, vol. 463 of Mathematics and its Applications. Dordrecht: Kluwer Academic Publishers, 1999.

[53] I.-G. E. Kordonis, N. T. Niyianni, and C. G. Philos, On the behavior of the solutions of scalar first order linear autonomous neutral delay 
differential equations, Arch. Math. (Basel), vol. 71, no. 6, pp. 454-464, 1998.

[54] M. A. Kaashoek; S. M. Verduyn Lunel, An integrability condition on the resolvent for hyperbolicity of the semigroup. J. Differential Equations 112 (1994), no. 2, 374-406.

[55] V. B. Kolmanovskii, V. R. Nosov, Stability of functional-differential equations. Mathematics in Science and Engineering, 180. Academic Press, Inc., London, 1986.

[56] J. Kurzweil, Generalized ordinary differential equations and continuous dependence on a parameter, Czech. Math. J., 7 (82), (1957), 418-448.

[57] J. Kurzweil, Generalized ordinary differential equations, Czechoslovak Math. J. 8(83) (1958), 360-388.

[58] Latushkin, Y.; Randolph, T.; Schnaubelt, R., Exponential dichotomy and mild solutions of nonautonomous equations in Banach spaces, J. Dynam. Differential Equations 10 (1998), no. 3, 489-510.

[59] J. C. Lillo, Periodic differential difference equations, J. Math. Anal. Appl., vol. 15, pp. 434-441, 1966.

[60] Massera, J. L.; Schäffer, J. J. Linear differential equations and functional analysis. I. Ann. of Math. (2) 671958 517-573.

[61] Massera, J. L.; Schäffer, J. J. Linear differential equations and function spaces. Pure and Applied Mathematics, Vol. 21 Academic Press, New York-London 1966

[62] Mă̌zel, A. D. On stability of solutions of systems of differential equations. Ural. Politehn. Inst. Trudy 51 (1954), 20-50.

[63] J. G. Mesquita, Método da média para equações diferenciais funcionais retardadas impulsivas via equações diferenciais generalizadas Dissertação de mestrado, 2009.

[64] F. Oliva, Z. Vorel, Functional equations and generalized ordinary differential equations, Bol. Soc. Mat. Mexicana, 11 (1966), 40-46. 
[65] A. D. Myshkis, Line $\breve{n y e ~ d i f f e r e n t s i a l ' n y e ~ u r a v n e n i y a ~ s ~ z a p a z d y v a y u s h-~}$ chim argumentom. Linear differential equations with retarded argument. Second edition. Izdat. "Nauka", Moscow, 1972.

[66] A. Pazy, Semigroups of linear operators and applications to partial differential equations. Applied Mathematical Sciences, 44. Springer-Verlag, New York, 1983.

[67] Perron, O. Die Stabilitätsfrage bei Differentialgleichungen. Math. Z. 32 (1930), no. 1, 703-728.

[68] C. G. Philos, Asymptotic behaviour, nonoscillation and stability in periodic first-order linear delay differential equations, Proc. Roy. Soc. Edinburgh Sect. A, vol. 128, no. 6, pp. 1371-1387, 1998.

[69] C. G. Philos, I. K. Purnaras, Periodic first order linear neutral delay differential equations, Appl. Math. Comput., vol. 117, no. 2-3, pp. 203$222,2001$.

[70] Preda, P.; Megan, M. Exponential dichotomy of evolutionary processes in Banach spaces. Czechoslovak Math. J. 35(110) (1985), no. 2, 312-323.

[71] Rodrigues, H. M.; Ruas-Filho, J. G. Evolution equations: dichotomies and the Fredholm alternative for bounded solutions. J. Differential Equations 119 (1995), no. 2, 263-283.

[72] H. L. Royden, Real analysis. Third edition. Macmillan Publishing Company, New York, 1988.

[73] W. Rudin, Real and complex analysis, Third edition. McGraw-Hill Book Co., New York, 1987.

[74] Š. Schwabik, Generalized Ordinary Differential Equations, Series in Real Anal., vol. 5, World Scientific, Singapore, 1992.

[75] Sacker, Robert J.; Sell, George R. Existence of dichotomies and invariant splittings for linear differential systems. I. J. Differential Equations 15 (1974), 429-458.

[76] Sacker, Robert J.; Sell, George R. Existence of dichotomies and invariant splittings for linear differential systems. II. J. Differential Equations 22 (1976), no. 2, 478-496. 
[77] Sacker, Robert J.; Sell, George R. Existence of dichotomies and invariant splittings for linear differential systems. III. J. Differential Equations 22 (1976), no. 2, 497-522.

[78] Sacker, Robert J.(1-SCA); Sell, George R.(1-MN-SM) Dichotomies for linear evolutionary equations in Banach spaces. J. Differential Equations 113 (1994), no. 1, 17-67.

[79] Sasu, A. L. Exponential dichotomy for evolution families on the real line. Abstr. Appl. Anal. 2006, Art. ID 31641, 16 pp.

[80] Sasu, A. L.; Sasu, B. Exponential dichotomy on the real line and admissibility of function spaces. Integral Equations Operator Theory 54 (2006), no. 1, 113-130

[81] R. M. Sievert, Zur theoretisch-mathematischen Behandlung des Problems der biologischen Strahlenwirkung. Acta Radiologica 22, (1941). $237-251$.

[82] A. Slavík, Dynamic equations on time scales and generalized ordinary differential equations, J. Math. Anal. Appl. 385 (2012), 534-550.

[83] A. Slavík, Measure functional differential equations with infinite delay, submitted.

[84] A. Stokes, A Floquet theory for functional differential equations. Proc. Nat. Acad. Sci. U.S.A., 48, 1962 1330-1334.

[85] A. E. Taylor, General theory of functions and integration. Blaisdell Publishing Co. Ginn and Co. New York-Toronto-London 1965

[86] V. Volterra, Sulle equazioni integrodifferenziali della teorie dell' elasticita. Atti Accad. Lincei (18), 295, 1909.

[87] V. Volterra, Sur la theorie mathemathique des phenomenes hereditaires, J. Math. Pures Appl. (7), 249-298, 1928.

[88] V. Volterra, Theorie mathemathique de la lute pour la vie, GauthierVillars, Paris, 1931. 


\section{Índice Remissivo}

$P_{\rho}$, projeção espectral, 69,78

Aplicação

atômica, 20

monodrômica, 27

Autoespaço generalizado, 66

Autovalor

de tipo finito, 68

dominante, 67

simples, 67

Dicotomia exponencial, 95

Divisão marcada, 51

$e^{a_{N} \omega}$, raio espectral de $T_{N}(s+\omega, s)$, 69

EDFN, 21

com parte neutra periódica, 82

em medida, 99

EDFR, 21

não autônoma, 89

não homogênea, 89

em medida, 101

Equação característica, 67

Espaço

sol estrela, 32

sol reflexivo, 34

Expoente característico, 28

Função

calibre, 52 escada finita, 58

regrada, 58

uniformemente não-atômica, 26

Gerador infinitesimal, 127

Integral

de Kurzweil, 51

fraca-*, 131

ker, núcleo de operador, 68

Multiplicador

característico, 28

de EDFN, 68

Multiplicidade

algébrica, 66

geométrica, 66

Operador

parte, 130

solução, 24

Perturbações limitadas

caso $\odot$-reflexivo, 42

com imagem de dimensão finita, 45

Im, imagem de operador, 68

Semigrupo

adjunto, 129

fortemente contínuo, 127 
Sistema de evolução, 86

fortemente contínuo, 87

orientado negativamente, 87 orientado positivamente, 87

Solução

continuação, 23

da EDO generalizada, 57 não-continuável, 23

$T(t, s)$, operador solução, 24

$T_{N}(t), 24,68$

$T_{N, f}(t), 24,68$ 